Universidade de São Paulo

Instituto de Física

\title{
Equações de estado do plasma de quarks e glúons e suas aplicações
}

\author{
Samuel Mendes Sanches Júnior
}

Orientador: Prof. Dr. Fernando Silveira Navarra

Tese de doutorado apresentada ao Instituto de Física para a obtenção do título de Doutor em Ciências.

Comissão Examinadora:

Prof. Dr. Fernando Silveira Navarra (orientador) (IFUSP)

Profa. Dra. Frederique Grassi (IFUSP)

Profa. Dra. Elizabeth Mara de Gouveia Dal Pino (IAG/USP)

Prof. Dr. Jorge Ernesto Horvath (IAG/USP)

Prof. Dr. Francisco Eugênio Mendonça da Silveira (UFABC)

São Paulo

2018 
FICHA CATALOGRÁFICA

Preparada pelo Serviço de Biblioteca e Informação do Instituto de Física da Universidade de São Paulo

Sanches Junior, Samuel Mendes

Equações de estado do plasma de quarks e glúons e suas aplicações. São Paulo, 2018.

Tese (Doutorado) - Universidade de São Paulo. Instituto de Física. Depto. de Física Experimental

Orientador: Prof. Dr. Fernando Silveira Navarra

Área de Concentração: Física de Partículas Elementares e Campos.

Unitermos: 1. Física de partículas; 2. Física moderna; 3. Física. 
Esta tese é dedicada aos meus queridos pais. Vocês são a inspiração que tenho para sempre ser uma pessoa melhor. Obrigado por tudo! Amo vocês! 


\section{Agradecimentos}

Mais uma etapa se encerra e não podemos esquecer de quem esteve presente nesta caminhada, apesar do fato de que escrever uma tese é um caminho solitário, o apoio e presença de muitas pessoas foram fundamentais para a finalização deste trabalho.

Agradeço à minha família por me incentivarem a sempre estudar. Principalmente aos meus pais, sempre me incentivando a seguir meus sonhos e apoiando emocionalmente e financeiramente, Cleuri e Samuel obrigado.

Aos meus irmãos e suas famílias, por estarem do nosso lado.

Ao professor Fernando Navarra pela amizade, orientação e risadas ao longo destes tantos anos.

Aos colegas, amigos, professores e funcionários do grupo de física de hádrons e física teórica, pelos cafés, conversas e incentivos. Ressaltando o amigo e colaborador Dr. David Fogaça pela amizade e trabalhos que realizamos em conjunto.

Aos amigos que sempre estiveram presentes para ouvir e aconselhar quando foi necessário.

À minha amada Thaís, que sempre me motivou mesmo nas horas mais difíceis com suas risadas e otimismo. Obrigado por me mostrar que podemos fazer o mundo melhor e que ao seu lado sou enfim completo.

O presente trabalho foi realizado com apoio da Coordenação de Aperfeiçoamento de Pessoal de Nível Superior - Brasil (CAPES) - Código de Financiamento 001. 


\section{Resumo}

O plasma de quarks e glúons é um assunto que vem sendo muito estudado nos últimos anos, devido ao advento dos colisores de partículas modernos e os avanços nas pesquisas relacionadas a estrelas compactas. Assim, nesta tese tivemos como objetivo principal o estudo deste plasma. Para isto, aprimoramos uma equação de estado desenvolvida pelo nosso grupo, na qual foi adicionada a interação com um campo magnético constante. De posse desta nova equação de estado, que é formulada a partir da técnica de aproximação de campo médio, fizemos diversas aplicações. Em particular, no estudo de propagação de ondas lineares e não lineares, com o objetivo de verificar se a causalidade e estabilidade são respeitadas. Resolvemos a equação de Tolman-Oppenheimer-Volkoff para obter o diagrama massa-raio para uma estrela de quarks compacta. Estudamos como é a evolução temporal do Universo primordial resolvendo as equações de Friedmann e a evolução temporal de bolhas do plasma de quarks e glúons no gás de hádrons (e também de bolhas de gás de hádrons no plasma de quarks e glúons) utilizando a equação de Rayleigh-Plesset relativística.

Palavras-chave: plasma de quarks e glúons, equação de estado, campo magnético. 


\section{Abstract}

The quark gluon plama is a subject that has been actively studied in recent years, due to the advent of modern particle colliders and advances in research related to compact stars. Thus, in this thesis we had as main goal the study of this plasma. For this, we improved an equation of state developed by our group, where an interaction with a constant magnetic field was added. With this new equation of state, which is formulated from the mean field approximation technique, we made several applications. As in the study of propagation of linear and non-linear waves, with the goal of verifying whether the causality and stability are respected. We solved the TolmanOppenheimer-Volkoff equation to obtain the mass-radius diagram for a compact quark star. We study how the temporal evolution of the primordial Universe by solving Friedmann's equations and the temporal evolution of bubbles of quark gluon plasma in a hadrons gas (and also of bubbles of hadrons gas in a quark gluon plasma) using the relativistic Rayleigh-Plesset equation.

Keywords: quark gluon plasma, equation of state, magnetic field. 


\section{Publicações}

Durante o período em que o doutorado foi realizado, os seguintes trabalhos foram submetidos à publicação em revistas indexadas ou como proceedings de conferências:

1. S. M. Sanches, F. S. Navarra and D. A. Fogaça, The quark gluon plasma equation of state and the expansion of the early Universe, Nucl. Phys. A 937, 1 (2015) [arXiv:1410.3893 [hep-ph]].

2. S. M. Sanches, D. A. Fogaça, F. S. Navarra and H. Marrochio, Cavitation in a quark gluon plasma with finite chemical potential and several transport coefficients, Phys. Rev. C 92, 025204 (2015) [arXiv:1505.06335 [hep-ph]].

3. D. A. Fogaça, F. S. Navarra and S. M. Sanches, Strongly coupled quark gluon plasma in a magnetic field, J. Phys. Conf. Ser. 630, 012027 (2015).

4. S. M. Sanches, D. A. Fogaça and F. S. Navarra, The time evolution of the quark gluon plasma in the early Universe, J. Phys. Conf. Ser. 630, 012028 (2015).

5. D. A. Fogaça, S. M. Sanches, R. Fariello and F. S. Navarra, Bubble dynamics and the quark-hadron phase transition in nuclear collisions, Phys. Rev. C 93, 055204 (2016) [arXiv:1601.04596 [hep-ph]].

6. D. A. Fogaça, S. M. Sanches, T. F. Motta and F. S. Navarra, Compact stars with strongly coupled quark matter in a strong magnetic field, Phys. Rev. C 94, 
055805 (2016) [arXiv:1608.00602 [hep-ph]].

7. D. A. Fogaça, S. M. Sanches and F. S. Navarra, Waves in magnetized quark matter, Nucl. Phys. A 973, 48 (2018) [arXiv:1706.02991 [hep-ph]].

8. D. A. Fogaça, S. M. Sanches and F. S. Navarra, Nonlinear waves in magnetized quark matter and the reduced Ostrovsky equation, Comm NonlinSci NumSim 66, 208 (2019) [arXiv:1802.01984 [nucl-th]]. 


\section{Conteúdo}

1 Introdução 1

1.1 Cromodinâmica Quântica . . . . . . . . . . . . . . . . . . 1

1.2 Plasma de Quarks e Glúons . . . . . . . . . . . . . . . . . . . . . 3

1.3 Equação de Estado . . . . . . . . . . . . . . . . . . . . . . 7

1.4 Organização da Tese . . . . . . . . . . . . . . . . . . . . . . 10

2 Equações de Estado do Plasma de Quarks e Glúons 11

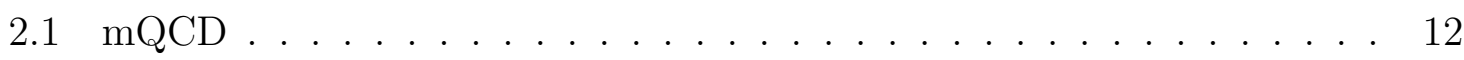

2.1.1 Decomposição do Campo dos Glúons e Condensados . . . . . 12

2.1 .2 Lagrangeana Efetiva . . . . . . . . . . . . . . . . . . 14

2.1 .3 O Campo Magnético . . . . . . . . . . . . . . . . . . 16

2.1.4 Equações de Movimento . . . . . . . . . . . . . . . . . . . 18

2.1 .5 Níveis de Landau . . . . . . . . . . . . . . . . . . . . . . . . . 19

2.1 .6 Equação de Estado . . . . . . . . . . . . . . . . . . . . . 21

2.2 Modelo de Sacola do MIT . . . . . . . . . . . . . . . . . . . . . . . 32

2.3 QCD na Rede . . . . . . . . . . . . . . . . . . . 34

3 Ondas no QGP $\quad 37$

3.1 Introdução . . . . . . . . . . . . . . . . . . . . . . . . . 37

3.2 Hidrodinâmica com Campo Magnético Externo . . . . . . . . . . . 38

3.3 Ondas Lineares . . . . . . . . . . . . . . . . . . . . . . . . . . . . 40

3.3 .1 Linearização . . . . . . . . . . . . . . . . . . . . . 40 
3.3 .2 Causalidade e Estabilidade . . . . . . . . . . . . . . . . . 41

3.4 Ondas Não Lineares . . . . . . . . . . . . . . . . . . . . 47

3.4.1 Método das Perturbações Redutivas (RPM) . . . . . . . . . . 47

3.4.2 Equação Reduzida de Ostrovsky . . . . . . . . . . . . . . . 50

3.5 Conclusão . . . . . . . . . . . . . . . . . . . . . 53

4 Aplicações $\quad 54$

4.1 Estrela de Quarks com Campo Magnético . . . . . . . . . . . . . . 54

4.1.1 Propriedades das Estrelas de Nêutrons . . . . . . . . . . . . 54

4.1 .2 Massa e Raio . . . . . . . . . . . . . . . . . . . . 57

4.1.3 Janela de Estabilidade e Diagrama Massa-Raio . . . . . . . . . 59

4.2 Universo Primordial . . . . . . . . . . . . . . . . 65

4.2 .1 Equações de Friedmann . . . . . . . . . . . . . . . . 66

4.2 .2 Evolução Temporal . . . . . . . . . . . . . . . . 67

4.3 Evolução de Bolhas de QGP em um Gás de Hádrons . . . . . . . . . . 76

4.3.1 Equação de Rayleigh-Plesset para um Fluido Relativístico . . . 78

4.3 .2 Limite Não Relativístico . . . . . . . . . . . . . . . . . . . . 80

4.3 .3 Temperatura Nula . . . . . . . . . . . . . 82

4.3.4 Temperatura Finita . . . . . . . . . . . . . . . 87

4.4 Conclusão . . . . . . . . . . . . . . . . . . . . . . . 89

5 Conclusões, Críticas e Perspectivas $\quad 90$

A Constantes de Estrutura de SU(3) 93

B Campo Fermiônico em Temperatura Finita 95

C Aproximações para Altas e Baixas Temperaturas 104

C.1 Altas temperaturas . . . . . . . . . . . . . . . . . 108

C.2 Baixas temperaturas . . . . . . . . . . . . . . 109 
D Solução Exata para a ROE

E Equação de Tolman-Oppenheimer-Volkoff (TOV)

F Equações de Friedmann

G Equação de Rayleigh-Plesset Relativística (RRP) e a Transformação de Sundman

G.1 Derivação da equação RRP . . . . . . . . . . . . . . . . . . . . . . . . 123

G.2 Transformação de Sundman . . . . . . . . . . . . . . . . . . . . . . . 129

Referências Bibliográficas 


\section{Lista de Figuras}

1.1 Diagrama de fase da QCD [14]. . . . . . . . . . . . . . . 4

1.2 Colisão frontal de núcleos pesados [23]. . . . . . . . . . . . . 5

1.3 Representação do interior de uma estrela de nêutrons. Devido ao seu pequeno tamanho $(\sim 10 \mathrm{~km})$, com uma massa da ordem de $2 M_{\odot}$, sua densidade é muito elevada $[29] . \ldots \ldots$. . . . . . . . . 6

1.4 Evolução do Universo primordial, com a escala de tempo (acima) e temperatura (abaixo) [30]. . . . . . . . . . . . 8

2.1 Diagramas de Feynman que representam as interações contidas na lagrangeana $(2.14) \ldots \ldots \ldots \ldots \ldots \ldots$

3.1 Comportamento solitônico da perturbação na densidade bariônica, $\left|\delta \rho_{B u}\right|$, para diversos tempos e intensidade de campo magnético. Usamos a equação de estado na aproximação de campo médio (mQCD) . . . . . . 52

4.1 Imagens em ótico, raios-X e representação gráfica de um pulsar, abaixo se tem a emissão regular de raios gamma com período de 0.0337s [127]. 55

4.2 Diagrama massa-raio para diferentes EOS [130]. . . . . . . . . . . . 58

4.3 Pressões paralela e perpendicular ao campo magnético. As linhas superiores mostram os resultados da $\operatorname{mQCD},\left(\xi=g / m_{G}\right)$ e as linhas inferiores os resultados obtidos com a EOS do MIT. . . . . . . . . . . . 59

4.4 Janela de estabilidade definidas pelas condições (4.1) à (4.5) . . . . . . . 60 
4.5 Diagrama de estabilidade: densidade bariônica (dividida por $\rho_{0}$ como função do campo magnético. A região cinza claro satisfaz (4.1), (4.3) e (4.4). A região em cinza escuro satisfaz também (4.5). . . . . . . . . . . 61

4.6 Curvas Massa-Raio obtidas com a EOS do MIT e da mQCD, para dois valores do campos magnético. $\mathcal{B}_{Q C D}$ e $\xi$ satisfazem o critério de estabilidade. A densidade central é $\rho_{B}=2.6 \rho_{0} \ldots \ldots \ldots \ldots$. . . . . . 62

4.7 Curvas Massa-Raio, obtidas com a mQCD variando $\mathcal{B}_{Q C D}$ e mantendo $\xi$ fixo.

4.8 Curvas Massa-Raio, obtidas com a mQCD, variando $\xi$ (MIT indica $\xi=0)$ e mantendo $\mathcal{B}_{Q C D}$ fixo.

4.9 Efeito da anisotropia das pressões sobre a massa da estrela calculada pela TOV. Os potenciais químicos satisfazem o critério de estabilidade e são: $\nu_{u}=300 \mathrm{MeV}, \nu_{d}=\nu_{s}=316.5 \mathrm{MeV}$ e $\mu_{e}=16.5 \mathrm{MeV} \ldots \ldots$.

4.10 Comparação das EOS do MIT e da mQCD. (a) Equação de estado. (b) Velocidade do som. . . . . . . . . . . . . . . . . . . . 68

4.11 Modelo de sacola do MIT, $\operatorname{com} \mathcal{B}=150 \mathrm{MeV} / \mathrm{fm}^{3}$. (a) Evolução temporal da densidade de energia. (b) Evolução temporal da temperatura.

4.12 Comparação da mQCD $\left(m_{g}=10 \mathrm{MeV}\right)$ com o MIT. (a) Razão da evolução temporal da densidade de energia. (b) Razão da evolução temporal da temperatura. . . . . . . . . . . . . . . 70

4.13 Resultados da EOS da LQCD. (a) Efeito do potencial químico na evolução temporal da densidade de energia. (b) Efeito do potencial químico na evolução temporal da temperatura. (c) Razão LQCD/MIT para densidade de energia. (d) Razão LQCD/MIT para temperatura. (e) Razão LQCD/mQCD para densidade de energia. (f) Razão LQCD/mQCD para temperatura. . . . . . . . . . . . . . . . . . 72

4.14 Contribuição EW para a evolução temporal com a EOS da LQCD. (a) Evolução temporal da densidade de energia. (b) Evolução temporal da

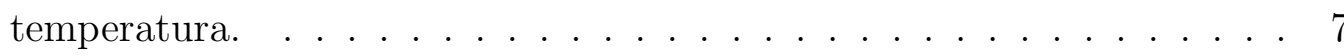


4.15 Fator de escala como função do tempo para os modelos MIT, LQCD (Rede) e mQCD $\left(m_{g}=10 \mathrm{MeV}\right) \ldots \ldots \ldots \ldots \ldots$

4.16 Esquema do estágio inicial de uma colisão em energias intermediárias. (a) Dois núcleos colidem. (b) Bolhas de QGP (Q) começam a se formar e crescer em um meio de hádrons $(\mathrm{H})$. (c) O restante de matéria hadrônica está dentro de bolhas que se contraem. No final o QGP é formado. .

4.17 Comparação entre as soluções relativística e não relativística da equação RP com altas densidades $\left(\rho_{B}>\rho_{c}\right)$. a) e b) expansão da bolha de QGP para diferentes EOS. c) e d) colapso da bolha de gás de hádrons para as mesmas duas EOS. . . . . . . . . . . . . . . . . 8

4.18 Tempo de evolução de uma bolha de QGP imersa em um gás de hádron. Soluções numéricas de (4.31) para diversas condições iniciais. Em todas situações: $\rho_{e x}=0.35 \mathrm{fm}^{-3}$ e $\rho_{i n}=1.0 \mathrm{fm}^{-3} \ldots \ldots \ldots \ldots$

4.19 Tempo de evolução de uma bolha de gás de hádrons imerso no QGP. Soluções numéricas de (4.31) para diversas condições iniciais. Em todas situações: $\rho_{e x}=0.6 \mathrm{fm}^{-3}$ e $\rho_{i n}=0.4 \mathrm{fm}^{-3} \ldots \ldots \ldots \ldots$. . . . 86

4.20 Tempo de evolução de uma bolha dada pela solução numérica de (4.31). Bolha de QGP com um raio inicial de $1 \mathrm{fm}$ (a) e $2 \mathrm{fm}$ (b) expandindo em um meio de gás de hádrons $\left(T_{e x}=140 \mathrm{MeV}\right.$ e $\left.T_{i n}=120 \mathrm{MeV}\right)$. Bolha de gás de hádrons de raio $1 \mathrm{fm}(\mathrm{c})$ e $2 \mathrm{fm}(\mathrm{d})$, em um meio de QGP $\left(T_{e x}=120 \mathrm{MeV}\right.$ e $\left.T_{i n}=138 \mathrm{MeV}\right) \ldots \ldots \ldots \ldots$ 


\section{Capítulo 1}

\section{Introdução}

Existem quatro forças fundamentais que regem a natureza: gravitacional, eletromagnética, fraca e forte. Dentre elas destacamos a forte que atua entre os quarks e glúons (mediadores da interação). Uma característica muito especial dos glúons é que eles possuem carga de cor. Como esta é a carga fundamental da interação forte, existem assim interações quark-quark, quark-glúon e também glúon-glúon. Todas elas são descritas pela teoria Cromodinâmica Quântica (Quantum Chromodynamics QCD) [1-7].

\subsection{Cromodinâmica Quântica}

A QCD é uma teoria de campos não abeliana invariante por transformações locais de gauge $\mathrm{SU}(3)$. Trabalhamos com três cores e as respectivas anticores $\left(N_{c}=3\right)$. Os quarks, os férmions da teoria, possuem spin 1/2, enquanto os glúons, os bósons da teoria, possuem spin 1.

Uma particularidade da teoria é o fato de que sua constante de acoplamento tende a zero $(g \rightarrow 0)$ em interações a curtas distâncias, ou equivalentemente, altas energias. Assim é possível utilizar métodos perturbativos para o cálculo das grandezas dese- 
jadas por meio da QCD perturbativa (perturbative QCD - pQCD). Entretanto, em interações a longas distâncias ou baixas energias a constante de acoplamento aumenta significantemente, e então os processos se tornam altamente não perturbativos. Esta propriedade marcante da teoria é a chamada liberdade assintótica [5-7].

A QCD também possui outra característica importante chamada de confinamento [8]. Estados observáveis devem ser singletos de cor e como quarks e glúons isolados não podem ser descritos como singletos de cor, não é possível que sejam observados livres na natureza. Os quarks e glúons estão permanentemente confinados no interior dos hádrons.

A lagrangeana da QCD é parecida com a da Eletrodinâmica Quântica (Quantum Electrodynamics - QED) [9-13]:

$$
\mathcal{L}=-\frac{1}{4} F_{\mu \nu}^{a} F^{a \mu \nu}+\bar{\psi}(\not D-m) \psi
$$

onde $\psi$ representa o campo dos quarks e é definido como:

$$
\psi=\left(\begin{array}{l}
\psi_{r} \\
\psi_{b} \\
\psi_{g}
\end{array}\right)
$$

onde $r$ (red), $b$ (blue) e $g$ (green) são índices de cor.

O termo $\not D$ é dado por:

$$
\not D=\gamma_{\mu} D^{\mu}=\gamma_{\mu}\left(\partial^{\mu}-i g G^{\mu}\right)=\gamma_{\mu}\left(\partial^{\mu}-i g G_{a}^{\mu} t_{a}\right)
$$

com o campo dos glúons sendo representado por $G_{a}^{\mu}, a=1,2, \ldots, 8 . t_{a}$ são os geradores do grupo $\mathrm{SU}(3)$ (matrizes hermiteanas, de traço nulo e dimensão $3 \times 3$ ). A letra $g$ representa a constante de acoplamento. 
O tensor do campo dos glúons $F^{a \mu \nu}$, é:

$$
F^{a \mu \nu}=\partial^{\mu} G^{a \nu}-\partial^{\nu} G^{a \mu}+g f^{a b c} G^{b \mu} G^{c \nu}
$$

onde $f^{a b c}$ são as constantes de estrutura do grupo $\mathrm{SU}(3)$ anti-simétricas pela troca de dois índices (ver Apêndice A).

A lagrangeana definida em (1.1) possui invariância sob as seguintes transformações de gauge:

$$
\begin{gathered}
\psi(x) \rightarrow \psi^{\prime}(x)=U(x) \psi(x) \\
\bar{\psi}(x) \rightarrow \bar{\psi}^{\prime}(x)=\bar{\psi}(x) U^{-1}(x) \\
G_{\mu}(x) \rightarrow G_{\mu}^{\prime}(x)=U(x) G_{\mu}(x) U^{-1}(x)-\frac{i}{g}\left(\partial_{\mu} U(x)\right) U^{-1}(x)
\end{gathered}
$$

com $U(x)=e^{i t^{a} \theta^{a}(x)}$ e $\theta^{a}(x)$ é um conjunto de funções reais e dependentes da posição e do tempo.

Apesar de a teoria ter sido testada diversas vezes como a teoria fundamental das interações fortes, nem todas as situações são bem entendidas. A hipótese de confinamento, ainda precisa ser provada matematicamente. Sistemas com densidades muito altas e baixas temperaturas (interior de estrelas compactas) e o nível de energia mais baixa da teoria (o vácuo da QCD) não são completamente entendidos.

Na Figura 1.1 mostramos o diagrama de fase da QCD.

\subsection{Plasma de Quarks e Glúons}

O estado da matéria denominado de plasma de quarks e glúons (Quark Gluon Plasma - QGP) ${ }^{1}$ é um gás de quarks e glúons desconfinados e podemos utilizar uma

\footnotetext{
${ }^{1}$ A denominação de plasma para o QGP, é usada porque a carga de cor que os quarks e glúons possuem $\left(q_{c}\right)$ é reduzida exponencialmente com a distância $(r)$ dividida pelo comprimento de Debye $(\alpha): q_{c} \rightarrow q_{c} e^{-r / \alpha}$. Este fenômeno é chamado de "screening" ou blindagem de cor. Um fenômeno semelhando ocorre em plasmas eletromagnéticos.
} 


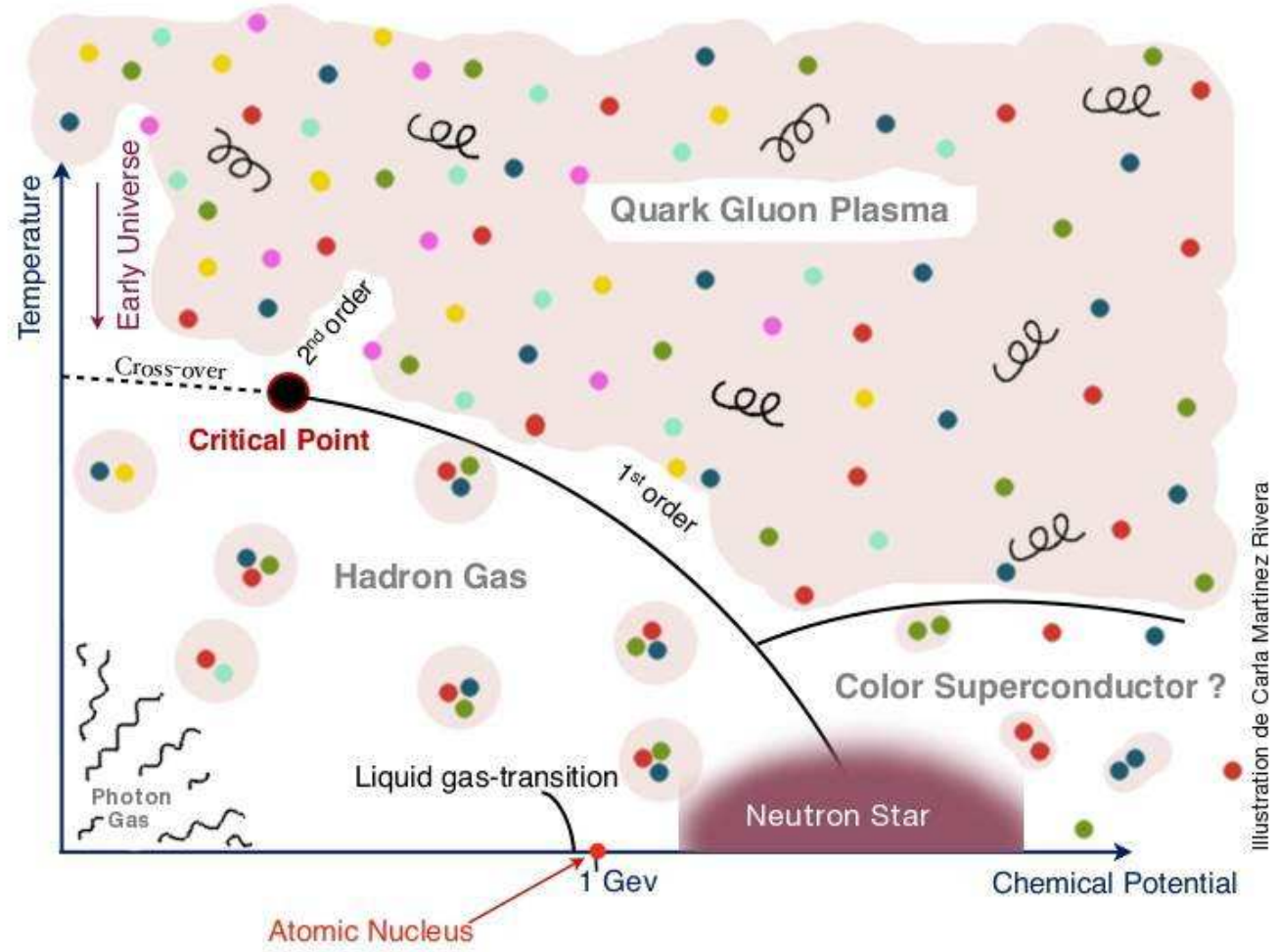

Figura 1.1: Diagrama de fase da QCD [14].

equação de estado (equation of state - EOS) para descrever suas propriedades macroscópicas tais como a pressão, densidade de energia, temperatura e entropia. A existência do QGP foi proposta inicialmente no final da década de 70 [15]. Ele era então considerado um fluido fracamente interagente, ideia que foi refutada mais tarde, quando se descobriu que o QGP é um fluido quase perfeito e fortemente interagente [16]. O QGP tem sido estudado especialmente em colisões de núcleos pesados relativísticos. Neste trabalho nós discutimos também os efeitos do QGP em estrelas compactas e em cosmologia na fase primordial do Universo.

\section{Colisões de núcleos pesados}

A produção do QGP em colisões de íons pesados foi confirmada no Relativistic 
Heavy Ion Collider (RHIC) localizado no Brookhaven National Laboratory [17-21] que utiliza núcleos de ouro com energia da ordem de $200 \mathrm{GeV}$ por par de nucleons no sistema do centro de massa. O QGP foi também estudado no Large Hadron Collider (LHC) localizado no CERN (Conseil Européen pour la Recherche Nucléaire) [22] que utiliza núcleos de chumbo com energia da ordem de $2.76 \mathrm{TeV}$ por par de nucleons no sistema do centro de massa.

Na Figura 1.2 mostramos uma colisão central de núcleos pesados. Inicialmente temos a representação pictórica dos dois núcleos (1.2a) que por possuírem velocidades relativísticas (próximas à velocidade da luz) aparecem contraídos, com formato parecido ao de uma "panqueca", vista no referencial do centro de massa da colisão. Em seguida ocorre a colisão (1.2b), onde os núcleos se superpõem, para então formarem um estado extremamente denso e quente, o QGP propriamente dito, (1.2c), que em $\sim 10-15 \mathrm{fm} / \mathrm{c}$ se expande e resfria (1.2d) coalescendo (ou se aglutinando) novamente nos hádrons (processo chamado de hadronização) e por fim produzindo hádrons que serão observados nos detectores (1.2e).

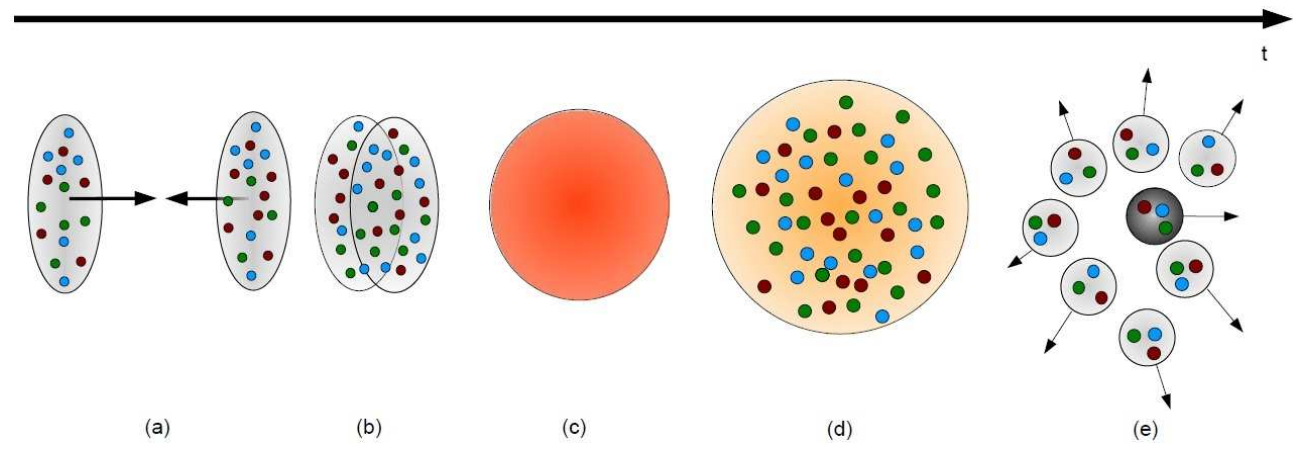

Figura 1.2: Colisão frontal de núcleos pesados [23].

\section{Estrelas compactas}

O termo estrelas compactas usualmente se refere a três objetos distintos: anãs brancas, estrelas de nêutrons e buracos negros. Estes são alguns dos possíveis estágios 
finais de uma estrela quando a pressão da radiação proveniente dos processos de fusão nuclear no seu interior não resiste mais à força gravitacional atrativa. Nesse ponto a estrela colapsa, restando um remanescente estelar muito denso. No caso das estrelas de nêutrons, devido à grande densidade em seu interior (da ordem de $10^{15} \mathrm{~g} / \mathrm{cm}^{3}$ ), podemos esperar que exista o QGP frio [24-28] como pode ser visto na Figura 1.3.

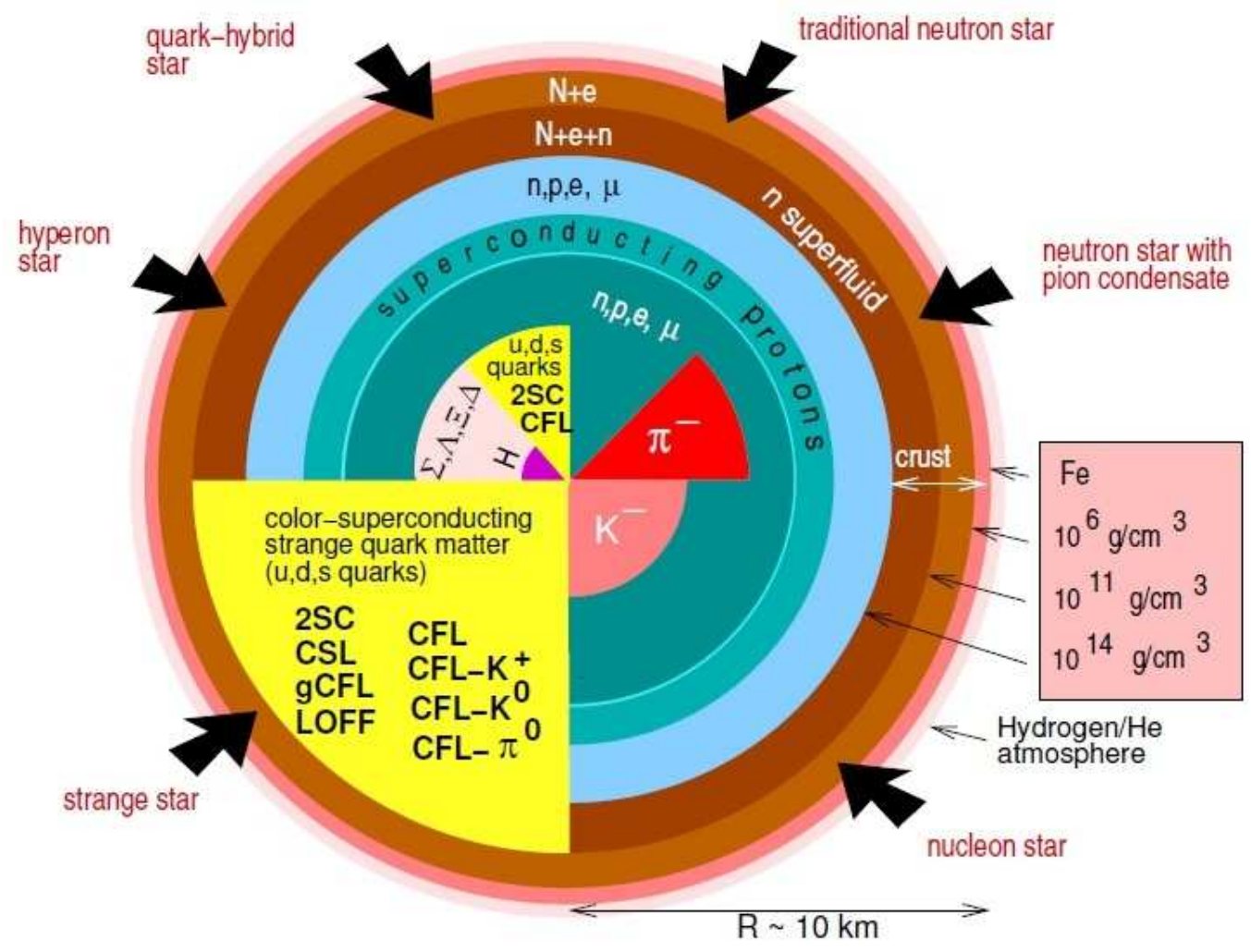

Figura 1.3: Representação do interior de uma estrela de nêutrons. Devido ao seu pequeno tamanho $(\sim 10 \mathrm{~km})$, com uma massa da ordem de $2 M_{\odot}$, sua densidade é muito elevada [29].

\section{Cosmologia}

O modelo do Big Bang prevê que a expansão do Universo ocorreu a partir de uma singularidade. Houve uma época em que a temperatura e a densidade eram tais que possibilitaram a existência do QGP. 
A evolução do Universo tem momentos importantes, que estão esquematizados na Figura 1.4, [31-33]:

- $\sim 10^{-43} \mathrm{~s}$ : Nesse período a temperatura era da ordem de $10^{19} \mathrm{GeV}$ (temperatura de Planck), o tamanho do Universo era o de uma partícula. Nesta época os efeitos da gravidade desempenharam papel crucial na evolução;

- $\sim 10^{-37} \mathrm{~s}$ : Com uma temperatura de $\sim 10^{15} \mathrm{GeV}$ houve uma quebra de simetria entre a interação eletrofraca e forte;

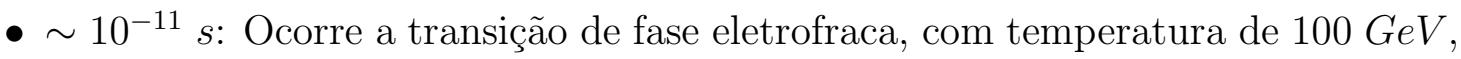
onde os léptons adquirem suas massas e os bósons da interação fraca e eletromagnénica se separam;

- $\sim 10^{-5} \mathrm{~s}$ : Com a temperatura mais baixa, $\sim 200 \mathrm{MeV}$, temos a transição quarkhádron, no período entre $\sim 10^{-10} \mathrm{~s}$ até $\sim 10^{-5} \mathrm{~s}$. O Universo passou então por um período composto de QGP;

- Após $\sim 10^{-5} \mathrm{~s}$ : O Universo segue esfriando, $<100 \mathrm{MeV}$, os quarks se combinam para formar os hádrons, seguindo depois para a combinação dos prótons e nêutrons em átomos leves $(\sim 1 \mathrm{keV} \mathrm{e} \sim 10 \mathrm{~s})$. Após $\sim 10^{13} \mathrm{~s}$ ou $\sim 3 \cdot 10^{5}$ anos ocorre a combinação dos elétrons com os núcleos e a emissão dos fótons que compõem a radiação cósmica de fundo, tornando o Universo transparente.

\subsection{Equação de Estado}

Para estudar teoricamente os problemas mencionados acima, envolvendo estrelas de nêutrons, cosmologia e colisões de íons pesados, precisamos usar termodinâmica e hidrodinâmica, que por sua vez, precisam de uma equação de estado que traduza em variáveis macroscópicas os graus de liberdade e a dinâmica das interações microscópicas. Nos cenários mencionados na seção anterior, estamos sempre lidando 


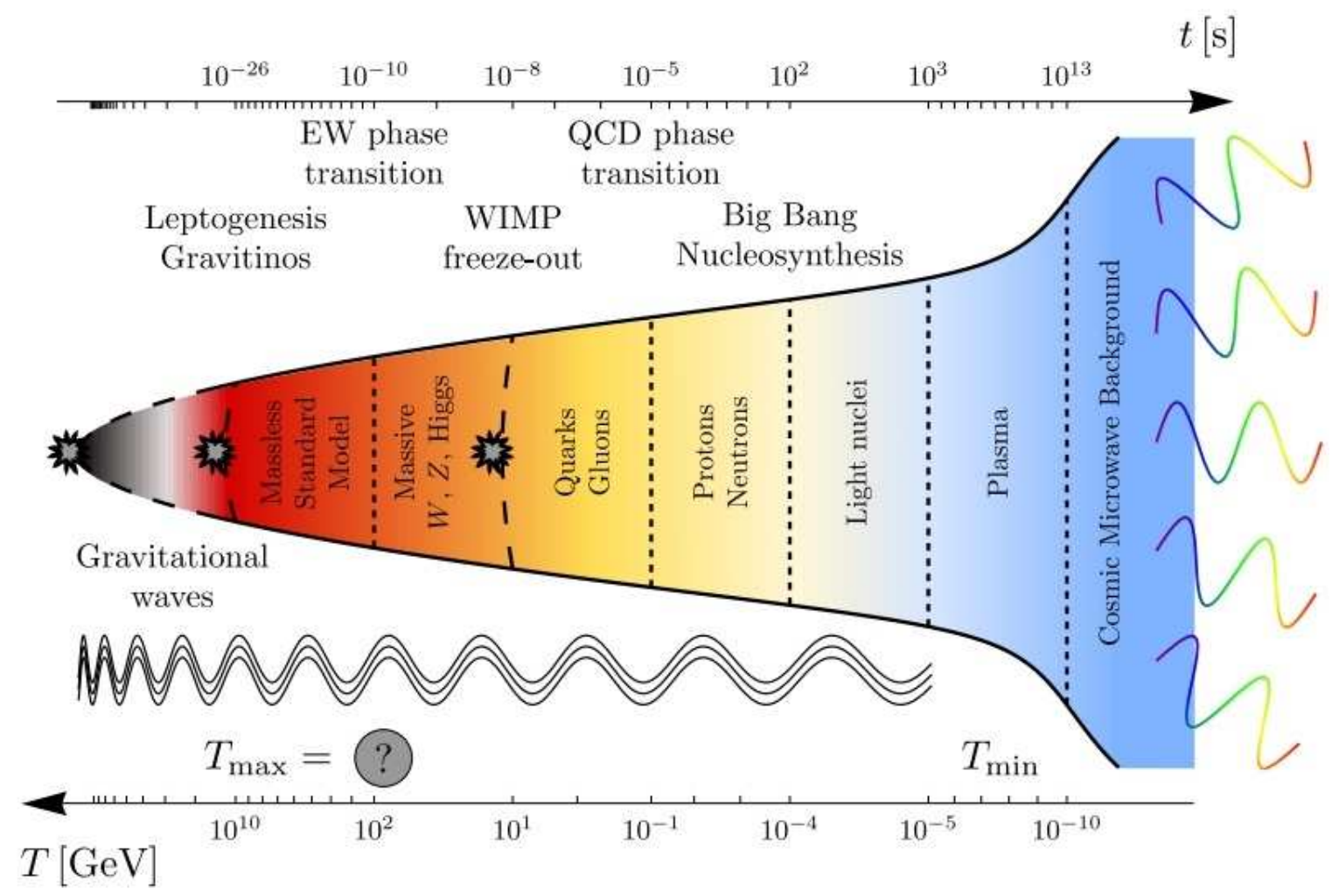

Figura 1.4: Evolução do Universo primordial, com a escala de tempo (acima) e temperatura (abaixo) [30].

com um fluido de quarks e glúons que num caso extremo (estrelas) está em repouso e é frio e no outro (colisões de íons pesados) está em expansão com uma velocidade próxima à da luz e a temperaturas altíssimas. O estudo da equação de estado do QGP, tanto frio como quente, e suas aplicações é o tema central desta tese. Estamos estudando uma EOS em particular, que vamos chamar de mQCD. Esta equação de estado foi desenvolvida para o estudo do plasma frio das estrelas compactas e seu maior sucesso, já nos primeiros testes, foi gerar uma pressão maior do que outras EOS que usavam quarks e glúons como graus de liberdade [34]. Mais tarde passou a ser usada em cosmologia e em colisões de íons pesados.

Neste ponto cabe a pergunta: "Por que fazer mais um modelo de equação de estado para o QGP?" Porque as propriedades coletivas da matéria existente no interior das estrelas de nêutrons são reconhecidamente difíceis de prever. Por um lado existe 
o chamado "problema do sinal" que inviabiliza os cálculos com QCD na rede [35]. Por outro lado, ferramentas como teorias quirais efetivas estão limitadas a densidades abaixo da densidade de saturação da matéria nuclear [36]. Além disso, a QCD perturbativa só se torna aplicável a densidades muito altas [37]. Nenhum cálculo controlável e de primeiros princípios é aplicável nas densidades encontradas no interior das estrelas de nêutrons.

Apesar das dificuldades é possível obter informações robustas sobre as propriedades da matéria de quarks superdensa e fria. É possível impor os limites estabelecidos por teorias quirais a baixas densidades, pela pQCD a altíssimas densidades e construir uma família de EOS termodinamicamente consistentes e que ainda satisfaçam a condição de atingir massas da ordem de duas massas solares. Como foi mostrado por Fraga, Kurkela e Vuorinem [38], a imposição destas condições reduz significativamente a liberdade na escolha da equação de estado.

No final do ano passado (2017) mais um dado importante surgiu. As colaborações Ligo e Virgo, observaram ondas gravitacionais emitidas pela fusão de duas estrelas de nêutrons [39]. Neste evento, antes da fusão, ocorre a deformação das estrelas de nêutrons, que altera a forma da frente de onda gravitacional. Este efeito pode ser quantificado em termos da "deformabilidade de maré", $\Lambda$, que é dada por [39, 40]:

$$
\Lambda=\operatorname{cte}\left[\frac{c^{2}}{G} \cdot \frac{R}{M}\right]^{5}
$$

Pela expressão acima vemos, que ao impor vínculos sobre o valor de $\Lambda$, os novos dados experimentais impõem também novos limites aos valores do raio $(R)$ e da massa $(M)$ de estrelas de nêutrons.

No extremo oposto do diagrama de fase da QCD, na região de baixas densidades bariônicas (e baixos potenciais químicos) e altas temperaturas, os cálculos feitos com QCD na rede são confiáveis e são ainda compatíveis com os dados experimentais de colisões nucleares realizadas no LHC.

Apesar das crescentes restrições impostas pelos avanços teóricos e experimentais, 
ainda restam muitas incertezas no nosso conhecimento da EOS do QGP e por isso novas equações de estado ou mudanças nas já existentes ainda são necessárias. Neste contexto, na presente tese estudamos o QGP em várias situações, desenvolvemos e testamos uma EOS que foi recentemente proposta em [41]. Ela foi formulada a partir da técnica de aproximação de campo médio e que será chamada ao longo do texto de mQCD (Mean Field QCD). Este aprimoramento consistiu em incluir o campo magnético nesta EOS e verificar quais são as consequências.

Ao propor uma nova equação de estado, devemos mostrar que ela "passa por todos os testes", ou seja, que ela reproduz os valores de pressão e densidade de energia onde eles tenham sido calculados com a QCD na rede (lattice QCD - LQCD) e os vínculos vindos das medidas de estrelas.

\subsection{Organização da Tese}

A tese está organizada como segue. No capítulo 2, apresentamos as equações de estado, que serão utilizadas no restante da tese. No capítulo 3, apresentamos um estudo de ondas no QGP com a utilização da mQCD, onde tanto ondas lineares como não lineares serão abordadas. No capítulo 4, mostramos estudos do QGP nos vários cenários possíveis: estrelas compactas, cosmologia e colisões de íons pesados.

Os resultados obtidos no Capítulo 3 sobre ondas lineares foram publicados na Ref. [42] e o trabalho sobre ondas não lineares na Ref. [43]. Os resultados do Capítulo 4 sobre a aplicação em estrelas compactas foram publicados na Ref. [44], sobre cosmologia foram publicados na Ref. [45] e sobre colisões de íons pesados foram publicados na Ref. [46]. Para manter a concisão e maior unidade da tese preferimos não incluir os resultados publicados na Ref. [47]. 


\section{Capítulo 2}

\section{Equações de Estado do Plasma de Quarks e Glúons}

Neste capítulo vamos apresentar as equações de estado que serão utilizadas no restante desta tese. Inicialmente vamos derivar a EOS da mQCD sem e com a presença de campo magnético. A seguir, mostramos os efeitos da temperatura e do campo magnético, discutindo o caso particular do campo muito intenso e também os limites de altas e baixas temperaturas com campo magnético nulo. Então apresentamos o modelo de sacola do MIT, que pode ser derivado como um caso limite da mQCD e por fim a equação de estado que é obtida através da QCD na rede (LQCD). Vale ressaltar que para a matéria relativística a EOS é uma relação entre a densidade de energia e a pressão. 


\section{$2.1 \quad \mathrm{mQCD}$}

Escrevendo a lagrangeana (1.1) de uma forma mais explícita, temos [34,41]:

$$
\mathcal{L}_{Q C D}=-\frac{1}{4} F_{\mu \nu}^{a} F^{a \mu \nu}+\sum_{q=1}^{N_{f}} \bar{\psi}_{i}^{q}\left[i \gamma^{\mu}\left(\delta_{i j} \partial_{\mu}-i g T_{i j}^{a} G_{\mu}^{a}\right)-\delta_{i j} m\right] \psi_{j}^{q}
$$

onde a soma em $q$ é feita sobre o número de sabores dos quarks $N_{f}$ e $F^{a \mu \nu}$ é dado por (1.4).

\subsubsection{Decomposição do Campo dos Glúons e Condensados}

No espaço dos momentos, o campo dos glúons pode ser decomposto na forma ${ }^{1}$ $[34,41,48,49]$ :

$$
G^{a \mu}(k)=A^{a \mu}(k)+\alpha^{a \mu}(k)
$$

onde $A^{a \mu}$ é a componente do campo que contém apenas baixos momentos ("soft gluons", responsáveis pelas interações de longo alcance e com características não perturbativas) e $\alpha^{a \mu}$ a componente de altos momentos ("hard gluons", que participam dos processos perturbativos).

No espaço de configuração, a equação (2.2), tem a forma:

$$
G^{a \mu}(x)=A^{a \mu}(x)+\alpha^{a \mu}(x)
$$

No limite de momentos muito pequenos o campo $A^{a \mu}(x)$ torna-se praticamente constante no espaço ${ }^{2}$ e dá origem aos condensados de glúons.

O QGP visto nas colisões de íons pesados produz matéria de quark desconfinada que ainda está em um regime de forte acoplamento e assim esperamos que os condensados

\footnotetext{
${ }^{1}$ Vamos omitir o "til" da transformada de Fourier.

${ }^{2}$ Como $A^{a \mu}(x)$ é a componente de baixos momentos (grande comprimento de onda), temos que $\partial^{\nu} A^{a \mu}(x) \cong 0$, pois $\int d^{4} k e^{i k \cdot x} A^{a \mu} k_{\nu} \cong 0$.
} 
de glúons sobrevivam neste sistema [50]. O valor não nulo destes condensados faz a grande diferença entre a mQCD e o modelo de sacola do MIT.

Vamos adotar a seguinte notação para os valores esperados de operadores $Y$ no QGP:

$$
\langle Q G P|Y| Q G P\rangle \equiv\langle Y\rangle
$$

Como nem o vácuo e nem o QGP possuem uma componente de cor privilegiada, temos que:

$$
\begin{gathered}
\left\langle A^{a \mu}\right\rangle=0 \\
\left\langle A^{a \mu} A^{b \nu} A^{c \rho}\right\rangle=0
\end{gathered}
$$

Os valores esperados não nulos serão aqueles com potências pares do campo $A^{a \mu}$. Eles são os parâmetros da mQCD. Por enquanto apenas vamos supor que eles sejam não nulos. Como mostrado explicitamente em $[48,49]$, eles podem ser escritos em termos de escalas de energia da seguinte maneira:

$$
\left\langle A^{a \mu} A^{b \nu}\right\rangle=-\frac{\delta^{a b}}{8} \frac{g^{\mu \nu}}{4} \mu_{0}^{2}
$$

$\mathrm{e}$

$$
\left\langle A_{\mu}^{a} A_{\nu}^{b} A^{c \rho} A^{d \eta}\right\rangle=\frac{\phi_{0}{ }^{4}}{(32)(34)}\left[g_{\mu \nu} g^{\rho \eta} \delta^{a b} \delta^{c d}+g_{\mu}{ }^{\rho} g_{\nu}{ }^{\eta} \delta^{a c} \delta^{b d}+g_{\mu}{ }^{\eta} g_{\nu}{ }^{\rho} \delta^{a d} \delta^{b c}\right]
$$

onde $\mu_{0}$ e $\phi_{0}$ são escalas de energia a serem determinadas fenomenologicamente. Como veremos a seguir, na aproximação de campo médio, surge na lagrangeana efetiva um termo de massa para os hard glúons. Esta massa é gerada através da interação dos hard glúons com os soft glúons que existem no vácuo (e possivelmente também no QGP) e é proporcional ao condensado $\left\langle A^{2}\right\rangle$. Seguindo $[48,49]$, definimos a massa dinâmica do glúon $m_{G}$ como:

$$
m_{G}^{2} \equiv \frac{9}{32} g^{2} \mu_{0}^{2}
$$




\subsubsection{Lagrangeana Efetiva}

Vamos derivar uma versão simplificada da teoria, que trata de matéria de quarks a temperatura nula, sem campo magnético, na qual todos os quarks, $u, d$ e $s$, têm a mesma massa $m$. Vamos considerar somente um sabor (ignorando a somatória sobre sabores) e no final introduzir um fator de degenerescência $\gamma=3$. Assim a lagrangeana (2.1) pode ser reescrita como:

$$
\mathcal{L}_{Q C D}=-\frac{1}{4} F_{\mu \nu}^{a} F^{a \mu \nu}+\bar{\psi}_{i}\left[i \gamma^{\mu}\left(\delta_{i j} \partial_{\mu}-i g T_{i j}^{a} G_{\mu}^{a}\right)-\delta_{i j} m\right] \psi_{j}
$$

Utilizando a decomposição do campo dos glúons (2.2) em (2.10) e em (1.4) e lembrando que $\partial^{\nu} A^{a \mu}(x)=0$ podemos escrever $\mathcal{L}_{Q C D}\left(G^{a \mu}=A^{a \mu}+\alpha^{a \mu}\right)=\mathcal{L}^{\prime}{ }_{Q C D}$, que é dada por:

$$
\mathcal{L}^{\prime}{ }_{Q C D}=-\frac{1}{4} F_{\mu \nu}^{a} F^{\prime a \mu \nu}+\bar{\psi}_{i}\left[i \gamma^{\mu}\left(\delta_{i j} \partial_{\mu}-i g T_{i j}^{a}\left(A^{a \mu}+\alpha^{a \mu}\right)\right)-\delta_{i j} m\right] \psi_{j}
$$

onde $F^{a \mu \nu}=F^{a \mu \nu}\left(G^{a \mu}=A^{a \mu}+\alpha^{a \mu}\right)$ é dado por:

$$
F^{\prime a \mu \nu}=g f^{a b c} A^{b \mu} A^{c \nu}+\Gamma^{a \mu \nu}+g f^{a b c} A^{b \mu} \alpha^{c \nu}+g f^{a b c} \alpha^{b \mu} A^{c \nu}
$$

com

$$
\Gamma^{a \mu \nu}=\partial^{\mu} \alpha^{a \nu}-\partial^{\nu} \alpha^{a \mu}+g f^{a b c} \alpha^{b \mu} \alpha^{c \nu}
$$

Escrevendo por extenso o termo $F^{\prime} F^{\prime}$ obtemos:

$$
\begin{gathered}
\mathcal{L}^{\prime}{ }_{Q C D}=-\frac{1}{4} \Gamma_{\mu \nu}^{a} \Gamma^{a \mu \nu}-\frac{g f^{a b c}}{2} \Gamma_{\mu \nu}^{a}\left(A^{b \mu} \alpha^{c \nu}+\alpha^{b \mu} A^{c \nu}+A^{b \mu} A^{c \nu}\right) \\
-\frac{g^{2} f^{a b c} f^{a d e}}{4}\left[A_{\mu}^{b} A_{\nu}^{c} A^{d \mu} A^{e \nu}\right. \\
+A_{\mu}^{b} A_{\nu}^{c} A^{d \mu} \alpha^{e \nu}+A_{\mu}^{b} A_{\nu}^{c} \alpha^{d \mu} A^{e \nu}+A_{\mu}^{b} \alpha_{\nu}^{c} A^{d \mu} A^{e \nu}+\alpha_{\mu}^{b} A_{\nu}^{c} A^{d \mu} A^{e \nu}
\end{gathered}
$$




$$
\begin{gathered}
\left.+A_{\mu}^{b} \alpha_{\nu}^{c} \alpha^{d \mu} A^{e \nu}+A_{\mu}^{b} \alpha_{\nu}^{c} A^{d \mu} \alpha^{e \nu}+\alpha_{\mu}^{b} A_{\nu}^{c} A^{d \mu} \alpha^{e \nu}+\alpha_{\mu}^{b} A_{\nu}^{c} \alpha^{d \mu} A^{e \nu}\right] \\
+\bar{\psi}_{i}\left\{i \gamma^{\mu}\left[\delta_{i j} \partial_{\mu}-i g T_{i j}^{a}\left(A^{a \mu}+\alpha^{a \mu}\right)\right]-\delta_{i j} m\right\} \psi_{j}
\end{gathered}
$$

Substituindo os termos envolvendo os soft glúons pelos seus valores esperados (2.5)(2.8), contraindo os tensores métricos, usando a anti-simetria das constantes de estruturas, usando (A.6) e escrevendo explicitamente o termo $\frac{1}{4} \Gamma_{\mu \nu}^{a} \Gamma^{a \mu \nu}$ encontramos:

$$
\begin{gathered}
\mathcal{L}_{Q C D}^{\prime}=-\frac{1}{2}\left[\left(\partial_{\mu} \alpha_{\nu}^{a}\right) \partial^{\mu} \alpha^{a \nu}-\left(\partial_{\nu} \alpha_{\mu}^{a}\right) \partial^{\mu} \alpha^{a \nu}\right]-\frac{1}{4}\left[\left(\partial_{\mu} \alpha_{\nu}^{a}-\partial_{\nu} \alpha_{\mu}^{a}\right) g f^{a b c} \alpha^{b \mu} \alpha^{c \nu}\right. \\
\left.+g f^{a b c} \alpha_{\mu}^{b} \alpha_{\nu}^{c}\left(\partial^{\mu} \alpha^{a \nu}-\partial^{\nu} \alpha^{a \mu}\right)\right]-\frac{g^{2} f^{a b c} f^{a d e}}{4} \alpha_{\mu}^{b} \alpha_{\nu}^{c} \alpha^{d \mu} \alpha^{e \nu}-\frac{9}{(4)(34)} g^{2} \phi_{0}{ }^{4}+\frac{9}{64} g^{2} \mu_{0}{ }^{2} \alpha_{\nu}^{a} \alpha^{a \nu} \\
+\bar{\psi}_{i}\left\{i \gamma^{\mu}\left[\delta_{i j} \partial_{\mu}-i g T_{i j}^{a} \alpha^{a \mu}\right]-\delta_{i j} m\right\} \psi_{j}
\end{gathered}
$$

Fazemos agora a aproximação de campo médio para os hard glúons, que pode ser assim resumida. Como a densidade de quarks é alta e o acoplamento entre os quarks e os hard glúons ainda não é suficientemente pequeno (pois estamos no sQGP), o campo de hard glúons é intenso e o número de ocupação dos estados deste campo é grande o suficiente para que ele seja tratado como um campo clássico. Fazemos assim uma aproximação do tipo Walecka $[51,52]$ que já foi utilizada anteriormente na QCD $[53,54]$ :

$$
\alpha_{\mu}^{a}=\alpha_{0}^{a} \delta_{\mu 0}
$$

sendo $\alpha_{0}^{a}=\alpha_{0}^{a}(\vec{x}, t)=$ constante. Neste trabalho as inomogeneidades do campo $\alpha$ não são relevantes e por isso tratamos o campo como constante no espaço e no tempo. Em outros trabalhos $[55,56]$, envolvendo propagação de ondas no QGP, as inomogeneidades foram consideradas e o campo $\alpha$ foi tratado como uma função das coordenadas.

A lagrangeana efetiva (2.15), torna-se finalmente:

$$
\mathcal{L}_{0}=\frac{m_{G}{ }^{2}}{2} \alpha_{0}^{a} \alpha_{0}^{a}-b \phi_{0}{ }^{4}+\sum_{f=u}^{d, s} \bar{\psi}_{i}^{f}\left(i \delta_{i j} \gamma^{\mu} \partial_{\mu}+g \gamma^{0} T_{i j}^{a} \alpha_{0}^{a}-\delta_{i j} m_{f}\right) \psi_{j}^{f}
$$


onde a constante $b$ é dada por

$$
b \equiv \frac{9}{4(34)} g^{2}
$$

Para melhor visualização das possíveis interações que esta teoria possui, apresentamos esquematicamente na Figura 2.1 os diagramas de Feynman derivados da lagrangeana (2.14). Nela linhas contínuas representam os quarks, as linhas tracejadas os hard glúons e as linhas helicoidais os soft glúons. Assim, os diagramas 2.1(a), 2.1(c), 2.1(e), 2.1(h) e 2.1(i) são nulos, pois possuem potências ímpares do campo $A^{a \mu}$ dos soft glúons. Já os diagramas 2.1(f) e 2.1(g) são nulos devido à simetria que a aproximação de campo médio para os hard glúons, expressão (2.16), introduz na lagrangeana. Encontramos produtos de tensores simétricos por tensores antissimétricos no espaço de cor como, por exemplo, $f^{a b c} \alpha_{0}^{b} \alpha_{0}^{c}(=0)$. O diagrama 2.1(d) se anula porque contém termos com $\partial_{\mu} A_{\nu}=0$. O diagrama 2.1(b) indica a interação entre os quarks e os hard glúons, 2.1(j) representa a geração da massa dinâmica $(2.9)$ e $2.1(\mathrm{k})$ o termo $b \phi_{0}^{4}$.

\subsubsection{O Campo Magnético}

Vamos agora introduzir um campo magnético clássico, com magnitude e sentido constante: $B=B \hat{z}$ (e então $A_{\mu}=(0, y B, 0,0)$ ) e também um outro campo espinorial para representar os elétrons. No estudo de estrelas compactas eles são necessários para garantir a neutralidade de carga [44].

A nova lagrangeana será dada por:

$$
\begin{gathered}
\mathcal{L}=-\frac{1}{4} F_{\mu \nu}^{a} F^{a \mu \nu}+\sum_{f=u}^{d, s} \bar{\psi}_{i}^{f}\left[i \gamma^{\mu}\left(\delta_{i j} \partial_{\mu}+i \delta_{i j} Q_{f} A_{\mu}-i g T_{i j}^{a} G_{\mu}^{a}\right)-\delta_{i j} m_{f}\right] \psi_{j}^{f} \\
-\frac{1}{16 \pi} F_{\mu \nu} F^{\mu \nu}+\bar{\psi}_{i}^{e}\left[i \gamma^{\mu}\left(\delta_{i j} \partial_{\mu}+i \delta_{i j} Q_{e} A_{\mu}\right)-\delta_{i j} m_{e}\right] \psi_{j}^{e}
\end{gathered}
$$

onde $Q_{f}$ é a carga de cada quark $f, Q_{e}$ é a carga do elétron e $m_{e}$ é a massa do elétron. 


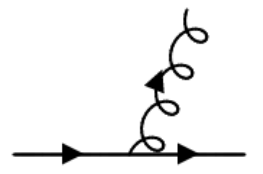

(a)

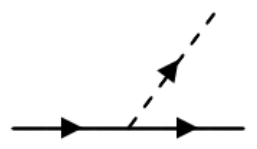

(b)

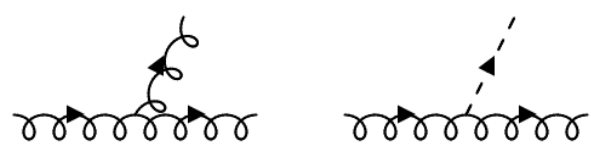

(c)

(d)

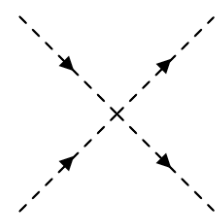

(g)

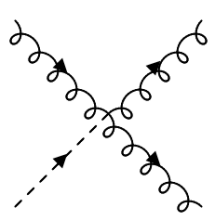

(h)

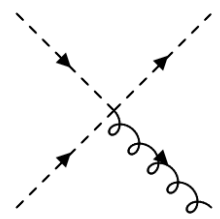

(i)

(e)

(f)
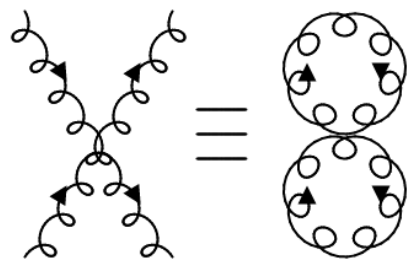

(j)

(k)

Figura 2.1: Diagramas de Feynman que representam as interações contidas na lagrangeana (2.14).

O termo eletromagnético será:

$$
\begin{gathered}
-\frac{1}{16 \pi} F_{\mu \nu} F^{\mu \nu}=-\frac{1}{16 \pi}\left[\partial^{\mu} A^{x}\left(\partial_{\mu} A_{x}\right)-\partial^{x} A^{x}\left(\partial_{x} A_{x}\right)-\partial^{x} A^{x}\left(\partial_{x} A_{x}\right)+\partial^{\nu} A^{x}\left(\partial_{\nu} A_{x}\right)\right] \\
=-\frac{1}{16 \pi}\left[2 \partial^{y} A^{x}\left(\partial_{y} A_{x}\right)\right]=-\frac{1}{16 \pi}\left[2 \partial^{y} A^{x}\left(\partial_{y} A_{x}\right)\right] \\
=-\frac{1}{8 \pi}\left[-\partial_{y}(-y B)\right]\left[\partial_{y}(y B)\right]=-\frac{B^{2}}{8 \pi}
\end{gathered}
$$

Substituindo a expressão acima na lagrangeana (2.19) encontramos:

$$
\mathcal{L}_{0}=\frac{m_{G}{ }^{2}}{2} \alpha_{0}^{a} \alpha_{0}^{a}-b \phi_{0}{ }^{4}-\frac{B^{2}}{8 \pi}+\bar{\psi}_{i}^{e}\left[i \gamma^{\mu}\left(\delta_{i j} \partial_{\mu}+i \delta_{i j} Q_{e} A_{\mu}\right)-\delta_{i j} m_{e}\right] \psi_{j}^{e}
$$




$$
+\sum_{f=u}^{d, s} \bar{\psi}_{i}^{f}\left\{i \gamma^{\mu}\left[\delta_{i j} \partial_{\mu}+i \delta_{i j} Q_{f} A_{\mu}\right]+g_{h} \gamma^{0} T_{i j}^{a} \alpha_{0}^{a}-\delta_{i j} m_{f}\right\} \psi_{j}^{f}
$$

\subsubsection{Equações de Movimento}

Vamos agora usar a lagrangeana (2.20) e as equações de Euler-Lagrange [57,58]:

$$
\frac{\partial \mathcal{L}}{\partial \eta_{i}}-\partial_{\mu} \frac{\partial \mathcal{L}}{\partial\left(\partial_{\mu} \eta_{i}\right)}=0
$$

sendo $\eta_{1}=\bar{\psi}^{f}, \eta_{2}=\bar{\psi}^{e}, \eta_{3}=\alpha_{0}^{a}, \eta_{4}=A^{\mu}$ e $\mathcal{L}=\mathcal{L}_{0}$. Encontramos as seguintes equações de movimento:

$$
\begin{gathered}
{\left[i \gamma^{\mu}\left(\partial_{\mu}+i Q_{f} A_{\mu}\right)+g_{h} \gamma^{0} T^{a} \alpha_{0}^{a}-m_{f}\right] \psi^{f}=0} \\
{\left[i \gamma^{\mu}\left(\partial_{\mu}+i Q_{e} A_{\mu}\right)-m_{e}\right] \psi^{e}=0} \\
m_{G}^{2} \alpha_{0}^{a}=-g_{h} \sum_{f=u}^{d, s} \rho_{f}^{a}=-g_{h} \rho^{a} \\
\partial_{\mu} F^{\mu \nu}=\sum_{f=u}^{d, s} Q_{f}\left(\bar{\psi}^{f} \gamma^{\nu} \psi^{f}\right)+Q_{e}\left(\bar{\psi}^{e} \gamma^{\nu} \psi^{e}\right)
\end{gathered}
$$

onde $\rho^{a}$ é a componente temporal do quadri-vetor densidade de corrente:

$$
j^{a \nu}=\sum_{f=u}^{d, s} \bar{\psi}_{i}^{f} \gamma^{\nu} T_{i j}^{a} \psi_{j}^{f}
$$

ou seja,

$$
j^{a 0}=\rho^{a}=\sum_{f=u}^{d, s} \bar{\psi}_{i}^{f} \gamma^{0} T_{i j}^{a} \psi_{j}^{f}=\sum_{f=u}^{d, s} \psi_{i}^{\dagger} T_{i j}^{a} \psi_{j}^{f}
$$




\subsubsection{Níveis de Landau}

\section{Solução Exata para os Elétrons}

Multiplicando a equação (2.23) por $\gamma^{0}$ pela esquerda, encontramos [59,60]:

$$
i \partial_{t} \psi=H_{\text {Dirac }} \psi
$$

onde

$$
H_{\text {Dirac }}=\sigma^{i}\left(-i \partial_{i}-Q_{e} A_{i}\right)+\gamma^{0} m_{e}
$$

Na expressão acima $\sigma^{i}=\gamma^{0} \gamma^{i}$ e $\gamma_{0}=\operatorname{diag}(1,1,-1,-1)$. O espinor $\psi$ pode ser escrito como:

$$
\psi=e^{i E t}\left(\begin{array}{l}
\phi \\
\xi
\end{array}\right)
$$

Substituindo (2.29) em (2.28) obtemos:

$$
\begin{aligned}
& (E-m) \phi=\sigma^{i}\left(-i \nabla_{i}-e Q A_{i}\right) \xi \\
& (E+m) \xi=\sigma^{i}\left(-i \nabla_{i}-e Q A_{i}\right) \phi
\end{aligned}
$$

Resolvendo o sistema de equações acima para $\phi$ encontramos:

$$
\left(E^{2}-m^{2}\right) \phi=\left[\sigma^{i}\left(-i \nabla_{i}-e Q A_{i}\right)\right]^{2} \phi
$$

Substituindo o potencial vetor em (2.32) temos:

$$
\left(E^{2}-m^{2}\right) \phi=\left[-\nabla^{2}+(e Q B)^{2} y^{2}-e Q B\left(2 i y \partial_{x}+\sigma_{3}\right)\right] \phi
$$

Vamos supor que a equação (2.33) admite soluções do tipo:

$$
\phi=e^{i \mathbf{k} \cdot \mathbf{X}} f(y)
$$


onde $f(y)$ é um vetor coluna de duas componentes e $X$ é um vetor espacial. Podemos ter duas soluções independentes:

$$
f_{+}=\left(\begin{array}{c}
F_{+}(y) \\
0
\end{array}\right) \text { e } f_{-}=\left(\begin{array}{c}
0 \\
F_{-}(y)
\end{array}\right)
$$

As funções $f$ devem ser autofunções de $\sigma_{3}$ com autovalores $s$. Assim, temos:

$$
\partial_{y}^{2} F_{s}=\left(q Q B y+k_{x}\right)^{2} F_{s}-\left(E^{2}-m^{2}-k_{z}^{2}+e Q B s\right) F_{s}
$$

que é uma equação de Hermite. Fazendo em (2.36) a mudança de variável:

$$
\chi=\sqrt{e|Q| B}\left(y+\frac{k_{x}}{e Q B}\right)
$$

a equação diferencial acima se transforma em:

$$
\left(\partial_{\chi}^{2}-\chi^{2}+a_{s} F_{s}\right)=0 \quad \text { com } \quad a_{s}=\frac{E^{2}-m^{2}-k_{z}^{2}+e Q B s}{e|Q| B}
$$

que admite os seguintes autovalores para a energia:

$$
E_{\nu}^{2}=m^{2}+k_{z}^{2}+(2 \nu+1) e|Q| B-e Q B s
$$

$\operatorname{com} \nu=0,1,2, \ldots$ representando os números de Hermite e $s= \pm 1$ o spin. Introduzindo agora o número de Landau, $2 \nu+1-s \times \operatorname{sgn}\left(Q_{f}\right)=2 n$, a energia dos eletrons é dada por

$$
E_{n}^{e( \pm)}= \pm \sqrt{m_{e}^{2}+k_{z}^{2}+2 n\left|Q_{e}\right| B}
$$

\section{Solução Exata para os Quarks}

A diferença entre a equação de movimento dos elétrons e a dos quarks, é que nesta última temos um novo termo constante na Hamiltoniana, $H \rightarrow H+H^{\prime}($ cte), que leva a $E \rightarrow E+E^{\prime}$. O espinor dos quarks possui um índice de cor, introduzido através de 
uma matriz $c_{j}\left(c_{i} c_{j}=\delta_{i j}\right)$ que multiplica (2.29). A energia é redefinida como:

$$
\tilde{E}_{\nu}^{f} \equiv E_{\nu}^{f}+g_{h} \mathcal{A}
$$

A energia quadrática dos quarks é dada por:

$$
\left(E_{\nu}^{f}+g_{h} \mathcal{A}\right)^{2}=m_{f}^{2}+k_{z}^{2}+(2 \nu+1)\left|Q_{f}\right| B-Q_{f} B s
$$

Finalmente, a energia dos quarks é:

$$
\tilde{E}_{n}^{f( \pm)}= \pm \sqrt{m_{f}^{2}+k_{z}^{2}+2 n\left|Q_{f}\right| B}
$$

onde o número de Landau é $2 \nu+1-s \times \operatorname{sgn}\left(Q_{f}\right)=2 n$.

\subsubsection{Equação de Estado}

Quando estudamos o plasma de quarks e glúons é necessário conhecer a equação de estado. Em geral tanto em colisões de íons pesados relativísticos como em estrelas compactas, temos matéria de quarks a temperatura finita e sob a ação de um campo magnético intenso.

Vamos derivar a equação de estado da mQCD no caso geral, i.e., a temperatura finita e com campo magnético. A seguir vamos considerar casos particulares que são mais relevantes para estudos fenomenológicos.

Primeiramente escrevemos a função de partição do sistema [56, 61, 62]:

$$
Z=\operatorname{Tr}\left\{\exp \left[-\left(\hat{H}-\mu_{e} \hat{N}_{e}-\sum_{f=u}^{d, s} \mu_{f} \hat{N}_{f}\right) / T\right]\right\}
$$

onde $\hat{H}$ é o operador Hamiltoniano, $\hat{N}_{e}$ é o operador número de elétrons (e pósitrons) e $\hat{N}_{f}$ é o operador número de quarks (e antiquarks). 
Como na aproximação de campo médio os campos bosônicos são tratados classicamente, o setor bosônico da função de partição, $Z_{b}$, se transforma numa função que não contém nenhum operador:

$$
Z_{b}=\exp \left[V\left(-\frac{m_{G}^{2}}{2} \alpha_{0}^{a} \alpha_{0}^{a}+b \phi_{0}^{4}+\frac{B^{2}}{8 \pi}\right) \frac{1}{T}\right]
$$

O setor fermiônico da função de partição deve ser tratado com o formalismo da teoria quântica de campos a temperatura finita, que está descrito no Apêndice B. A função de partição completa, é:

$$
\begin{gathered}
Z=\exp \left[V\left\{-\frac{m_{G}{ }^{2}}{2} \alpha_{0}^{a} \alpha_{0}^{a}+b \phi_{0}{ }^{4}+\frac{B^{2}}{8 \pi}\right\} \frac{1}{T}\right] \times \\
\times \prod_{\vec{k}, s, n}\left\{1+\exp \left[-\left(\mathcal{E}_{n}^{e}-\mu_{e}\right) / T\right]\right\}\left\{1+\exp \left[-\left(\mathcal{E}_{n}^{e}+\mu_{e}\right) / T\right]\right\} \\
\times \prod_{f=u}^{d, s} \prod_{\vec{k}, s, n}\left\{1+\exp \left[-\left(\mathcal{E}_{n}^{f}-\nu_{f}\right) / T\right]\right\}\left\{1+\exp \left[-\left(\mathcal{E}_{n}^{f}+\nu_{f}\right) / T\right]\right\}
\end{gathered}
$$

onde as energias são dadas por:

$$
\begin{aligned}
\mathcal{E}_{n}^{e} & =\sqrt{m_{e}^{2}+k_{z}^{2}+2 n\left|Q_{e}\right| B} \\
\mathcal{E}_{n}^{f} & =\sqrt{m_{f}^{2}+k_{z}^{2}+2 n\left|Q_{f}\right| B}
\end{aligned}
$$

e $\mu_{e}$ é o potencial químico dos elétrons. O potencial químico efetivo do quark $f$ é dado por:

$$
\nu_{f} \equiv \mu_{f}+g_{h}\left(c_{i}^{\dagger} T_{i j}^{a} c_{j}\right) \alpha_{0}^{a}
$$




\section{Pressão, Entropia e Energia}

Para encontrar as quantidades termodinâmicas, partimos do potencial termodinâmico, que é dado por:

$$
\Omega=-T \ln (Z)
$$

Usando os resultados da subseção anterior obtemos:

$$
\begin{gathered}
\Omega=\left[-\frac{m_{G}{ }^{2}}{2} \alpha_{0}^{a} \alpha_{0}^{a}+b \phi_{0}{ }^{4}+\frac{B^{2}}{8 \pi}\right] V \\
-T \sum_{\vec{k}, s, n}\left\{\ln \left[1+e^{-\left(\mathcal{E}_{n}^{e}-\mu_{e}\right) / T}\right]+\ln \left[1+e^{-\left(\mathcal{E}_{n}^{e}+\mu_{e}\right) / T}\right]\right\} \\
-T \sum_{f=u}^{d, s} \sum_{\vec{k}, s, n}\left\{\ln \left[1+e^{-\left(\mathcal{E}_{n}^{f}-\nu_{f}\right) / T}\right]+\ln \left[1+e^{-\left(\mathcal{E}_{n}^{f}+\nu_{f}\right) / T}\right]\right\}
\end{gathered}
$$

A pressão paralela à direção do campo magnético é [62-64]:

$$
p_{\|}=-\frac{\Omega}{V}
$$

isto é:

$$
\begin{gathered}
p_{\|}=\frac{3 g_{h}{ }^{2}}{2 N m_{G}{ }^{2}} \rho^{2}-b \phi_{0}{ }^{4}-\frac{B^{2}}{8 \pi}+\frac{\left|Q_{e}\right| B}{2 \pi^{2}} \sum_{n}\left(2-\delta_{n 0}\right) \int_{0}^{\infty} d k_{z} \frac{k_{z}{ }^{2}}{\mathcal{E}_{n}^{e}}\left(d_{e}+\bar{d}_{e}\right) \\
+\sum_{f=u}^{d, s} \frac{\left|Q_{f}\right| B}{2 \pi^{2}} \sum_{n} 3\left(2-\delta_{n 0}\right) \int_{0}^{\infty} d k_{z} \frac{k_{z}{ }^{2}}{\mathcal{E}_{n}^{f}}\left(d_{f}+\bar{d}_{f}\right)
\end{gathered}
$$

onde

$$
d_{i} \equiv \frac{1}{1+e^{\left(\mathcal{E}_{n}^{i}-\nu_{i}\right) / T}}
$$

e

$$
\bar{d}_{i} \equiv \frac{1}{1+e^{\left(\mathcal{E}_{n}^{i}+\nu_{i}\right) / T}}
$$


são as distribuições de férmions e antiférmions respectivamente. Para chegar à expressão (2.53) utilizamos o limite do contínuo na soma dos momentos. Isto foi feito porque as órbitas de Landau são helicoidais e quando projetadas no plano $z$ se tornam circulares. Assim, de acordo com [65,66], o momento transversal se torna discreto e $\propto 2 \nu$ que é um número inteiro proporcional ao número de Landau $(2 n)$ :

$$
k_{\perp}^{2}=2 \nu|Q| B
$$

Então a integral no momento se transforma em:

$$
\int d^{3} k \rightarrow 2 \nu|Q| B
$$

Como a soma é também sobre os spins, é necessário incluir um fator de degenerescência que depende do nível de Landau. Assim, o limite do contínuo é:

$$
\frac{1}{V} \sum_{\vec{k}, s, n} \longrightarrow \frac{1}{(2 \pi)^{3}} \sum_{n} \gamma_{i}(n) \int d^{3} k=\frac{\left|Q_{i}\right| B}{(2 \pi)^{2}} \sum_{n} \gamma_{i}(n) \int_{-\infty}^{\infty} d k_{z}
$$

onde $\gamma_{i}(n)$ é o fator estatístico do $i$-ésimo férmion. Para o elétron temos $\gamma_{e}(n)=$ $\left(2-\delta_{n 0}\right)$ e para cada quark $f$ temos $\gamma_{f}(n)=3\left(2-\delta_{n 0}\right)$, sendo que o fator 3 vem da soma sobre as três cores e o fator 2 vem da soma sobre os dois estados do spin.

As manipulações algébricas para derivar as quantidades termodinâmicas podem ser encontradas em detalhe no Apêndice B. A magnetização é dada por:

$$
M=-\frac{1}{V} \frac{\partial \Omega}{\partial B}=\frac{\partial p_{\|}}{\partial B}
$$

Com o auxílio de (2.53) reescrevemos a expressão acima como:

$$
M=-\frac{B}{4 \pi}-T \frac{\left|Q_{e}\right|}{2 \pi^{2}} \sum_{n}\left(2-\delta_{n 0}\right) \int_{0}^{\infty} d k_{z}\left[\ln \left(1-d_{e}\right)+\ln \left(1-\bar{d}_{e}\right)\right]
$$




$$
\begin{gathered}
-T \sum_{f=u}^{d, s} \frac{\left|Q_{f}\right|}{2 \pi^{2}} \sum_{n} 3\left(2-\delta_{n 0}\right) \int_{0}^{\infty} d k_{z}\left[\ln \left(1-d_{f}\right)+\ln \left(1-\bar{d}_{f}\right)\right] \\
-\frac{\left|Q_{e}\right| B}{2 \pi^{2}} \sum_{n}\left(2-\delta_{n 0}\right) \int_{0}^{\infty} d k_{z}\left[\frac{d_{e} n\left|Q_{e}\right|}{\mathcal{E}_{n}^{e}}+\frac{\bar{d}_{e} n\left|Q_{e}\right|}{\mathcal{E}_{n}^{e}}\right] \\
-\sum_{f=u}^{d, s} \frac{\left|Q_{f}\right| B}{2 \pi^{2}} \sum_{n} 3\left(2-\delta_{n 0}\right) \int_{0}^{\infty} d k_{z}\left[\frac{d_{f} n\left|Q_{f}\right|}{\mathcal{E}_{n}^{f}}+\frac{\bar{d}_{f} n\left|Q_{f}\right|}{\mathcal{E}_{n}^{f}}\right]
\end{gathered}
$$

A pressão perpendicular ao campo, $p_{\perp}$, é dada por [67]:

$$
p_{\perp}=p_{\|}-M B
$$

e pode ser escrita como:

$$
\begin{gathered}
p_{\perp}=\frac{3 g_{h}{ }^{2}}{2 N m_{G}{ }^{2}} \rho^{2}-b \phi_{0}{ }^{4}+\frac{B^{2}}{8 \pi}+\frac{\left|Q_{e}\right| B^{2}}{2 \pi^{2}} \sum_{n}\left(2-\delta_{n 0}\right) \int_{0}^{\infty} d k_{z}\left[\frac{d_{e} n\left|Q_{e}\right|}{\mathcal{E}_{n}^{e}}+\frac{\bar{d}_{e} n\left|Q_{e}\right|}{\mathcal{E}_{n}^{e}}\right] \\
+\sum_{f=u}^{d, s} \frac{\left|Q_{f}\right| B^{2}}{2 \pi^{2}} \sum_{n} 3\left(2-\delta_{n 0}\right) \int_{0}^{\infty} d k_{z}\left[\frac{d_{f} n\left|Q_{f}\right|}{\mathcal{E}_{n}^{f}}+\frac{\bar{d}_{f} n\left|Q_{f}\right|}{\mathcal{E}_{n}^{f}}\right]
\end{gathered}
$$

A densidade do quark f, $\rho_{f}=\frac{\partial p_{\perp}}{\partial \mu_{f}}$, é:

$$
\rho=\sum_{f=u}^{d, s} \frac{\left|Q_{f}\right| B}{2 \pi^{2}} \sum_{n} 3\left(2-\delta_{n 0}\right) \int_{0}^{\infty} d k_{z}\left(d_{f}-\bar{d}_{f}\right)
$$

e a densidade de elétrons, $\rho_{e}=\frac{\partial p_{\perp}}{\partial \mu_{e}}$, é escrita como:

$$
\rho_{e}=\frac{\left|Q_{e}\right| B}{2 \pi^{2}} \sum_{n}\left(2-\delta_{n 0}\right) \int_{0}^{\infty} d k_{z}\left(d_{e}-\bar{d}_{e}\right)
$$

A densidade de entropia, $s=\frac{\partial p_{\perp}}{\partial T}$, é:

$$
s=-\frac{\left|Q_{e}\right| B}{2 \pi^{2}} \sum_{n}\left(2-\delta_{n 0}\right) \int_{0}^{\infty} d k_{z}\left\{d_{e} \ln \left(d_{e}\right)+\left(1-d_{e}\right) \ln \left(1-d_{e}\right)\right.
$$




$$
\begin{gathered}
\left.+\bar{d}_{e} \ln \left(\bar{d}_{e}\right)+\left(1-\bar{d}_{e}\right) \ln \left(1-\bar{d}_{e}\right)\right\} \\
-\sum_{f=u}^{d, s} \frac{\left|Q_{f}\right| B}{2 \pi^{2}} \sum_{n} 3\left(2-\delta_{n 0}\right) \int_{0}^{\infty} d k_{z}\left\{d_{f} \ln \left(d_{f}\right)+\left(1-d_{f}\right) \ln \left(1-d_{f}\right)\right. \\
\left.+\bar{d}_{f} \ln \left(\bar{d}_{f}\right)+\left(1-\bar{d}_{f}\right) \ln \left(1-\bar{d}_{f}\right)\right\}
\end{gathered}
$$

A densidade de energia, $\varepsilon$, é calculada a partir da relação de Gibbs:

$$
\varepsilon=-p_{\|}+T s+\mu_{e} \rho_{e}+\sum_{f=u}^{d, s} \mu_{f} \rho_{f}
$$

resultando em:

$$
\begin{gathered}
\varepsilon=\frac{3 g_{h}{ }^{2}}{2 N m_{G}^{2}} \rho^{2}+b \phi_{0}^{4}+\frac{B^{2}}{8 \pi}+\frac{\left|Q_{e}\right| B}{2 \pi^{2}} \sum_{n}\left(2-\delta_{n 0}\right) \int_{0}^{\infty} d k_{z} \mathcal{E}_{n}^{e}\left(d_{e}+\bar{d}_{e}\right) \\
+\sum_{f=u}^{d, s} \frac{\left|Q_{f}\right| B}{2 \pi^{2}} \sum_{n} 3\left(2-\delta_{n 0}\right) \int_{0}^{\infty} d k_{z} \mathcal{E}_{n}^{f}\left(d_{f}+\bar{d}_{f}\right)
\end{gathered}
$$

Assim, as expressões (2.53), (2.60) e (2.65), são as equações de estado gerais para o QGP, na aproximação da mQCD.

\section{Temperatura Nula}

Pela simplicidade e pela relevância no contexto de astrofísica vamos agora considerar o limite das expressões acima para o caso em que a temperatura é nula, já estudado em [61,62,64-66]. Neste limite, as distribuição são:

$$
d_{i}=\Theta\left(\nu_{i}-\mathcal{E}_{n}^{i}\right) \quad \quad \text { e } \quad \bar{d}_{i}=0
$$


e também [61]:

$$
\lim _{T \rightarrow 0} T \ln \left(1-d_{i}\right)=\left(\mathcal{E}_{n}^{i}-\nu_{i}\right) \quad \text { e } \quad \lim _{T \rightarrow 0} T \ln \left(1-\bar{d}_{i}\right)=0
$$

A densidade de quarks será:

$$
\begin{gathered}
\rho=\sum_{f=u}^{d, s} \frac{\left|Q_{f}\right| B}{(2 \pi)^{2}} \sum_{n=0}^{n_{\text {max }}^{f}} 3\left(2-\delta_{n 0}\right) \int_{-\infty}^{\infty} d k_{z} \Theta\left(\nu_{f}-\mathcal{E}_{n}^{f}\right) \\
=\sum_{f=u}^{d, s} \frac{\left|Q_{f}\right| B}{2 \pi^{2}} \sum_{n=0}^{n_{\text {max }}^{f}} 3\left(2-\delta_{n 0}\right) \int_{0}^{k_{z, F}} d k_{z}=\sum_{f=u}^{d, s} \frac{\left|Q_{f}\right| B}{2 \pi^{2}} \sum_{n=0}^{n_{\text {max }}^{f}} 3\left(2-\delta_{n 0}\right) k_{z, F}^{f}(n)
\end{gathered}
$$

As distribuições (2.66) implicam:

$$
\sqrt{m_{f}^{2}+k_{z, F}^{f}{ }^{2}+2 n\left|Q_{f}\right| B}=\nu_{f} \quad \rightarrow \quad k_{z, F}^{f}=\sqrt{\nu_{f}^{2}-m_{f}^{2}-2 n\left|Q_{f}\right| B}
$$

O momento em (2.69) tem que ser positivo, o que implica a existência de um valor máximo para os níveis de Landau:

$$
n \leq n_{\text {max }}^{f}=\frac{\nu_{f}^{2}-m_{f}^{2}}{2\left|Q_{f}\right| B}
$$

Analogamente, para os elétrons temos:

$$
\rho_{e}=\frac{\left|Q_{e}\right| B}{2 \pi^{2}} \sum_{n=0}^{n_{\max }^{e}}\left(2-\delta_{n 0}\right) k_{z, F}^{e}(n)
$$

com

$$
k_{z, F}^{e}=\sqrt{\mu_{e}^{2}-m_{e}^{2}-2 n\left|Q_{e}\right| B}
$$

e

$$
n \leq n_{\max }^{e}=\frac{\mu_{e}^{2}-m_{e}^{2}}{2\left|Q_{e}\right| B}
$$


A densidade de energia e pressão são dadas por:

$$
\begin{aligned}
& \varepsilon=\frac{3 g_{h}{ }^{2}}{2 N m_{G}{ }^{2}} \rho^{2}+b \phi_{0}{ }^{4}+\frac{B^{2}}{8 \pi}+\frac{\left|Q_{e}\right| B}{2 \pi^{2}} \sum_{n=0}^{n_{\max }^{e}}\left(2-\delta_{n 0}\right) \int_{0}^{k_{z, F}^{e}} d k_{z} \sqrt{m_{e}^{2}+k_{z}^{2}+2 n\left|Q_{e}\right| B} \\
& +\sum_{f=u}^{d, s} \frac{\left|Q_{f}\right| B}{2 \pi^{2}} \sum_{n=0}^{n_{\max }^{f}} 3\left(2-\delta_{n 0}\right) \int_{0}^{k_{z, F}^{f}} d k_{z} \sqrt{m_{f}^{2}+k_{z}^{2}+2 n\left|Q_{f}\right| B} \\
& p_{\|}=\frac{3 g_{h}{ }^{2}}{2 N m_{G}{ }^{2}} \rho^{2}-b \phi_{0}{ }^{4}-\frac{B^{2}}{8 \pi}+\frac{\left|Q_{e}\right| B}{2 \pi^{2}} \sum_{n=0}^{n_{\max }^{e}}\left(2-\delta_{n 0}\right) \int_{0}^{k_{z, F}^{e}} d k_{z} \frac{k_{z}{ }^{2}}{\sqrt{m_{e}^{2}+k_{z}^{2}+2 n\left|Q_{e}\right| B}} \\
& +\sum_{f=u}^{d, s} \frac{\left|Q_{f}\right| B}{2 \pi^{2}} \sum_{n=0}^{n_{\max }^{f}} 3\left(2-\delta_{n 0}\right) \int_{0}^{k_{z, F}^{f}} d k_{z} \frac{k_{z}{ }^{2}}{\sqrt{m_{f}^{2}+k_{z}^{2}+2 n\left|Q_{f}\right| B}} \\
& p_{\perp}=\frac{3 g_{h}{ }^{2}}{2 N m_{G}{ }^{2}} \rho^{2}-b \phi_{0}{ }^{4}+\frac{B^{2}}{8 \pi}+\frac{\left|Q_{e}\right|^{2} B^{2}}{2 \pi^{2}} \sum_{n=0}^{n_{\max }^{e}}\left(2-\delta_{n 0}\right) n \int_{0}^{k_{z, F}^{e}} \frac{d k_{z}}{\sqrt{m_{e}^{2}+k_{z}^{2}+2 n\left|Q_{e}\right| B}} \\
& +\sum_{f=u}^{d, s} \frac{\left|Q_{f}\right|^{2} B^{2}}{2 \pi^{2}} \sum_{n=0}^{n_{\max }^{f}} 3\left(2-\delta_{n 0}\right) n \int_{0}^{k_{z, F}^{f}} \frac{d k_{z}}{\sqrt{m_{f}^{2}+k_{z}^{2}+2 n\left|Q_{f}\right| B}}
\end{aligned}
$$

As expressões (2.74), (2.75) e (2.76), são as equações de estado para o QGP, na aproximação de temperatura nula, com campo magnético.

Temperatura Nula e Campo Magnético Intenso: As componentes $p_{\|}$e $p_{\perp}$ são especialmente diferentes quando o campo magnético é muito intenso. Isso pode ser melhor entendido nos casos em que $B>\left(\nu_{f}^{2}-m_{f}^{2}\right) / 2\left|Q_{f}\right|$ e também $B>>$ $\left(\mu_{e}-m_{e}^{2}\right) / 2\left|Q_{e}\right|$. Nestas condições $(2.70)$ e (2.73) são:

$$
\lim _{B \rightarrow \infty} n \leq \lim _{B \rightarrow \infty} n_{\max }=\lim _{B \rightarrow \infty} \frac{\left(\nu_{f}^{2}-m_{f}^{2}\right)}{2\left|Q_{f}\right| B}=\frac{\left(\mu_{e}^{2}-m_{e}^{2}\right)}{2\left|Q_{e}\right| B}=0
$$


e consequentemente apenas o menor nível de Landau $(n=0)$ contribui para os níveis de Fermi do quark e do elétron:

$$
k_{z, F}^{f(B \rightarrow \infty)}=\sqrt{\nu_{f}^{2}-m_{f}^{2}} \quad e^{e(B \rightarrow \infty)}=\sqrt{\mu_{e}^{2}-m_{e}^{2}}
$$

e portanto:

$$
\begin{gathered}
\varepsilon_{(B \rightarrow \infty)}=\frac{3 g_{h}{ }^{2}}{2 N m_{G}{ }^{2}} \rho^{2}+b \phi_{0}{ }^{4}+\frac{B^{2}}{8 \pi}+\frac{\left|Q_{e}\right| B}{2 \pi^{2}} \int_{0}^{k_{z, F}^{e(B \rightarrow \infty)}} d k_{z} \sqrt{m_{e}^{2}+k_{z}^{2}} \\
+\sum_{f=u}^{d, s} \frac{3\left|Q_{f}\right| B}{2 \pi^{2}} \int_{0}^{k_{z, F}^{f(B \rightarrow \infty)} d k_{z} \sqrt{m_{f}^{2}+k_{z}^{2}}} \\
p_{\|(B \rightarrow \infty)}=\frac{3 g_{h}{ }^{2}}{2 N m_{G}^{2}} \rho^{2}-b \phi_{0}{ }^{4}-\frac{B^{2}}{8 \pi}+\frac{\left|Q_{e}\right| B}{2 \pi^{2}} \int_{0}^{k_{z, F}^{e(B \rightarrow \infty)}} d k_{z} \frac{k_{z}^{2}}{\sqrt{m_{e}^{2}+k_{z}^{2}}} \\
+\sum_{f=u}^{d, s} \frac{3\left|Q_{f}\right| B}{2 \pi^{2}} \int_{0}^{k_{z, F}^{f(B \rightarrow \infty)}} d k_{z} \frac{k_{z}^{2}}{\sqrt{m_{f}^{2}+k_{z}^{2}}} \\
p_{\perp(B \rightarrow \infty)}=\frac{3 g_{h}^{2}}{2 N m_{G}^{2}} \rho^{2}-b \phi_{0}^{4}+\frac{B^{2}}{8 \pi}
\end{gathered}
$$

As expressões (2.79), (2.80) e (2.81), são as equações de estado para o QGP, na aproximação de temperatura nula, com campo magnético intenso.

\section{Temperatura Finita com Campo Magnético Nulo}

Outro caso particular de interesse é o de temperatura finita e campo magnético nulo, que pode ser relevante no estudo do sQGP formado em colisões de íons pesados relativísticos. Neste caso, a função de partição pode ser obtida fazendo $B=0 \mathrm{em}$ 
$(2.44)$ e $(2.45)$ :

$$
\begin{gathered}
Z=\exp \left[V\left\{-\frac{m_{G}^{2}}{2} \alpha_{0}^{a} \alpha_{0}^{a}+b \phi_{0}^{4}\right\} \frac{1}{T}\right] \times \\
\times \prod_{\vec{k}, s, n}\left\{1+\exp \left[-\left(\mathcal{E}_{e}-\mu_{e}\right) / T\right]\right\}\left\{1+\exp \left[-\left(\mathcal{E}_{e}+\mu_{e}\right) / T\right]\right\} \\
\times \prod_{f=u}^{d, s} \prod_{\vec{k}, s, n}\left\{1+\exp \left[-\left(\mathcal{E}_{f}-\nu_{f}\right) / T\right]\right\}\left\{1+\exp \left[-\left(\mathcal{E}_{f}+\nu_{f}\right) / T\right]\right\}
\end{gathered}
$$

com as energias:

$$
\mathcal{E}_{e}=\sqrt{m_{e}^{2}+k^{2}} \quad e \quad \mathcal{E}_{f}=\sqrt{m_{f}^{2}+k^{2}}
$$

O potencial termodinâmico é dado por:

$$
\begin{gathered}
\Omega=\left[-\frac{m_{G}{ }^{2}}{2} \alpha_{0}^{a} \alpha_{0}^{a}+b \phi_{0}^{4}\right] V \\
+T \sum_{\vec{k}, s}\left\{\ln \left(1-d_{e}\right)+\ln \left(1-\bar{d}_{e}\right)\right\}+T \sum_{f=u}^{d, s} \sum_{\vec{k}, s}\left\{\ln \left(1-d_{f}\right)+\ln \left(1-\bar{d}_{f}\right)\right\}
\end{gathered}
$$

Para calcular a pressão, usamos [61]:

$$
\Omega=-p V=E-T S-\mu_{e} N_{e}-\sum_{f=u, d}^{s} \mu_{f} N_{f} \quad \text { então } \quad p=-\frac{\Omega}{V}
$$

Como antes, o limite do contínuo é encontrado fazendo:

$$
\frac{1}{V} \sum_{\vec{k}, s} \longrightarrow \frac{\gamma_{i}}{(2 \pi)^{3}} \int d^{3} k
$$

onde $\gamma_{i}$ é o fator estatístico do $i$-ésimo férmion. A pressão é obtida através de:

$$
p=\frac{3 g_{h}{ }^{2}}{2 N m_{G}^{2}} \rho^{2}-b \phi_{0}{ }^{4}+\frac{\gamma_{e}}{6 \pi^{2}} \int_{0}^{\infty} d k \frac{k^{4}}{\sqrt{m_{e}^{2}+k^{2}}}\left(d_{e}+\bar{d}_{e}\right)
$$




$$
+\sum_{f=u}^{d, s} \frac{\gamma_{f}}{6 \pi^{2}} \int_{0}^{\infty} d k \frac{k^{4}}{\sqrt{m_{f}^{2}+k^{2}}}\left(d_{f}+\bar{d}_{f}\right)
$$

As densidades de quarks e elétrons são respectivamente:

$$
\rho_{f}=\sum_{f=u}^{d, s} \frac{\gamma_{f}}{(2 \pi)^{3}} \int d^{3} k\left(d_{f}-\bar{d}_{f}\right) \quad e \quad \rho_{e}=\frac{\gamma_{e}}{(2 \pi)^{3}} \int d^{3} k\left(d_{e}-\bar{d}_{e}\right)
$$

A densidade de entropia é dada por:

$$
\begin{gathered}
s=-\frac{\gamma_{e}}{(2 \pi)^{3}} \int d^{3} k\left\{d_{e} \ln \left(d_{e}\right)+\left(1-d_{e}\right) \ln \left(1-d_{e}\right)+\bar{d}_{e} \ln \left(\bar{d}_{e}\right)+\left(1-\bar{d}_{e}\right) \ln \left(1-\bar{d}_{e}\right)\right\} \\
-\sum_{f=u}^{d, s} \frac{\gamma_{f}}{(2 \pi)^{3}} \int d^{3} k\left\{d_{f} \ln \left(d_{f}\right)+\left(1-d_{f}\right) \ln \left(1-d_{f}\right)\right. \\
\left.+\bar{d}_{f} \ln \left(\bar{d}_{f}\right)+\left(1-\bar{d}_{f}\right) \ln \left(1-\bar{d}_{f}\right)\right\}
\end{gathered}
$$

e a densidade de energia é dada por:

$$
\begin{gathered}
\varepsilon=\frac{3 g_{h}{ }^{2}}{2 N m_{G}^{2}} \rho^{2}+b \phi_{0}^{4}+\frac{\gamma_{e}}{2 \pi^{2}} \int_{0}^{\infty} d k k^{2} \sqrt{m_{e}^{2}+k^{2}}\left(d_{e}+\bar{d}_{e}\right) \\
+\sum_{f=u}^{d, s} \frac{\gamma_{f}}{2 \pi^{2}} \int_{0}^{\infty} d k k^{2} \sqrt{m_{f}^{2}+k^{2}}\left(d_{f}+\bar{d}_{f}\right)
\end{gathered}
$$

As expressões (2.87) e (2.90), são as equações de estado para o QGP, na aproximação de temperatura finita, sem campo magnético.

Altas Temperaturas e Campo Magnético Nulo: O limite de altas temperaturas, pode ser entendido como $T>>\nu_{i}$ e $T>>m_{i}$. No Apêndice C.1 estão todos os detalhes e as aproximações necessárias para encontrar as grandezas termodinâmicas. 
Listamos abaixo as expressões obtidas para as densidades dos férmions:

$$
\rho_{f(T \text { alta })}=\sum_{f=u}^{d, s} \frac{\gamma_{f}}{6} T^{2} \nu_{f} \quad e \quad \rho_{e(T \text { alta })}=\frac{\gamma_{e}}{6} T^{2} \mu_{e}
$$

para a densidade de energia:

$$
\begin{gathered}
\varepsilon_{(T \text { alta })=\frac{3 g_{h}{ }^{2}}{2 N m_{G}{ }^{2}}} \frac{T^{4}}{6^{2}}\left(\sum_{f=u}^{d, s} \gamma_{f} \nu_{f}\right)^{2}+b \phi_{0}{ }^{4}+\gamma_{e} \frac{7 \pi^{2}}{120} T^{4}+\frac{\gamma_{e}}{4} T^{2} \mu_{e}{ }^{2} \\
+\sum_{f=u}^{d, s} \gamma_{f} \frac{7 \pi^{2}}{120} T^{4}+\sum_{f=u}^{d, s} \frac{\gamma_{f}}{4} T^{2} \nu_{f}^{2}
\end{gathered}
$$

e para a pressão:

$$
\begin{gathered}
p_{(\text {T alta })=\frac{3 g_{h}{ }^{2}}{2 N m_{G}{ }^{2}}} \frac{T^{4}}{6^{2}}\left(\sum_{f=u}^{d, s} \gamma_{f} \nu_{f}\right)^{2}-b{\phi_{0}}^{4}+\gamma_{e} \frac{7 \pi^{2}}{360} T^{4}+\frac{\gamma_{e}}{12} T^{2} \mu_{e}{ }^{2} \\
+\sum_{f=u}^{d, s} \gamma_{f} \frac{7 \pi^{2}}{360} T^{4}+\sum_{f=u}^{d, s} \frac{\gamma_{f}}{12} T^{2} \nu_{f}{ }^{2}
\end{gathered}
$$

As expressões (2.92) e (2.93), são as equações de estado para o QGP, na aproximação de altas temperaturas, sem campo magnético.

\subsection{Modelo de Sacola do MIT}

O primeiro "modelo de sacola" foi proposto em 1967 em [70]. Naquele modelo os quarks tinham massa $m$ e estavam confinados em um volume esférico de raio $R$. A atração confinante entre eles vinha de um campo escalar. Dentro do volume esférico os quarks poderiam se mover livremente mas permaneceriam confinados. Mesmo sendo um modelo extremamente simples, ele foi capaz de gerar previsões realistas [71]. 
O modelo de sacola mais popular foi o do MIT, proposto em 1974 [72]. Ele conseguiu resolver, entre outros, o problema de violação da conservação da energia que o modelo de 1967 apresentava. Isto foi possível com a introdução de uma "pressão de confinamento" fenomenológica oriunda de um termo constante incluído na lagrangeana: o termo de sacola $\mathcal{B}$. Ele foi também crucial para a reprodução de diversas outras propriedades dos nucleons [73].

A estrutura interna de um hádron está associada aos campos dos glúons e dos quarks que o compõem. Embora a abordagem para descrever estas partículas seja convencional em teoria de campos, os campos que descrevem os quarks nos hádrons não estão em todos os pontos do espaço, mas apenas em seu interior (no interior da sacola). O conjunto destes pontos é chamado de "bag", à qual é atribuida a propriedade de confinamento, visto que a carga de cor não pode escapar do seu interior. Assim, hádrons podem ser vistos como uma bolha no vácuo não perturbativo da QCD. A pressão e a densidade de energia (com quarks de massas iguais $m_{q}$ ) e glúons são respectivamente dadas por:

$$
p_{M I T}(T, \mu)=\sum_{q=u}^{d, s} \frac{\gamma_{q}}{6 \pi^{2}} \int_{0}^{\infty} d k \frac{k^{4}}{\mathcal{E}_{q}}\left(d_{q}+\bar{d}_{q}\right)+\frac{\gamma_{g}}{6 \pi^{2}} \int_{0}^{\infty} d k k^{3}\left(e^{k / T}-1\right)^{-1}-\mathcal{B}
$$

e:

$$
\varepsilon_{M I T}(T, \mu)=\sum_{q=u}^{d, s} \frac{\gamma_{q}}{2 \pi^{2}} \int_{0}^{\infty} d k k^{2} \mathcal{E}_{q}\left(d_{q}+\bar{d}_{q}\right)+\frac{\gamma_{g}}{2 \pi^{2}} \int_{0}^{\infty} d k k^{3}\left(e^{k / T}-1\right)^{-1}+\mathcal{B}
$$

onde, como visto antes, os férmions obedecem às distribuições:

$$
d_{q} \equiv \frac{1}{1+e^{\left(\mathcal{E}_{q}-\mu\right) / T}} \quad \quad \text { e } \quad \bar{d}_{q} \equiv \frac{1}{1+e^{\left(\mathcal{E}_{q}+\mu\right) / T}}
$$

onde a energia de cada quark é dada por $\mathcal{E}_{q}=\sqrt{m_{q}^{2}+k^{2}}$ e $\mu$ é o potencial químico dos quarks e os fatores estatísticos são $\gamma_{g}=2$ (polarizações $) \times 8($ cores $)=16$ para os glúons e $\gamma_{q}=2$ (spins $) \times 3$ (cores $)=6$ para cada sabor de quark. 
Modelos fenomenológicos conseguem reproduzir o espectro dos hádrons. Entretando a relação entre eles e a QCD ainda é pouco clara e derivá-los a partir de uma lagrangeana efetiva pode ajudar neste entendimento. Comparando as expressões para o $\operatorname{MIT}(2.94)$ e (2.95), e as da mQCD (2.87) e (2.90), vemos que no caso do MIT o termo de sacola $\mathcal{B}$ aparece subtraído na pressão e somado na densidade de energia, e na mQCD o termo correspondente à componente gluônica aparece, também, subtraído na pressão e somado na densidade de energia. Assim, podemos fazer a relação:

$$
\mathcal{B}=b \phi_{0}^{4}=\left\langle\frac{1}{4} F^{a \mu \nu} F_{\mu \nu}^{a}\right\rangle=\mathcal{B}_{Q C D}
$$

este novo termo é uma espécie de "constante de sacola da QCD".

\subsection{QCD na Rede}

Um dos métodos mais bem estabelecidos para uma abordagem não perturbativa da QCD é a QCD na rede [74,75]. Um resultado com alta precisão obtido por meio dela foi o espectro dos hádrons leves [76]. Este método também apresenta algumas limitações. Em primeiro lugar ele precisa de uma capacidade de cálculo numérico/computacional muito alta. Além disso, em QCD na rede fazemos os cálculos no espaço Euclidiano. A função de partição do sistema é escrita inicialmente no espaço-tempo de Minkowski, mas a seguir introduzimos o tempo imaginário através da transformação $t \rightarrow i \tau$, ou seja:

$$
Z=\int D \phi e^{i S[\phi]} \rightarrow \int D \phi e^{-S_{E}[\phi]}
$$

onde $\phi$ representa o conjunto de campos da teoria descritos pela ação $S$. Com a transfomação, a métrica passa a ser Euclidiana e $S_{E}$ a ação Euclidiana. Esta transformação pode trazer problemas, pois nem todos os observáveis podem ser calculados em tempos imaginários. Além disso, encontramos o chamado "problema do sinal" [35] que aparece quando incluímos o potencial químico $\mu$. Neste caso (que é muito rele- 
vante no estudo de matéria de quarks a altas densidades) a ação da expressão (2.98) recebe um novo termo que não é hermitiano e assim o peso da exponencial acabará sendo complexo! A inclusão de potencial químico é um problema bastante estudado e existem diversas tentativas de resolvê-lo, cada uma com seus pontos fortes e fracos, como pode ser visto em [77-84]. A seguir, vamos mostrar uma parametrização que inclui potencial químico via expansão em Taylor dos observáveis termodinâmicos. A desvantagem deste método é restringir o estudo para potenciais químicos não muito grandes [85].

Uma parametrização muito útil é a obtida em $[85,86]$. Ela é feita com temperatura e potencial químico finitos, com três sabores de quarks $(u, d$ e $s)$ com massas iguais e glúons e é dada por:

$$
\begin{gathered}
\frac{\varepsilon(T, \mu)-3 p(T, \mu)}{T^{4}}=T \frac{\partial}{\partial T}\left[\frac{p(T, \mu)}{T^{4}}\right]+\frac{\mu^{2}}{T^{2}} \chi_{2} \\
=\frac{\varepsilon(T, 0)-3 p(T, 0)}{T^{4}}+\frac{\mu^{2}}{2 T} \frac{\partial \chi_{2}}{\partial T}
\end{gathered}
$$

onde a combinação de variáveis $\frac{\varepsilon-3 p}{T^{4}}$ é chamada de "anomalia do traço" e $\chi_{2}$ é o termo do coeficiente da expansão em série de Taylor. Para potencial químico nulo, temos a parametrização $[85,86]$ :

$$
\frac{\varepsilon(T, 0)-3 p(T, 0)}{T^{4}}=e^{-h_{1} / \tau-h_{2} / \tau^{2}}\left[h_{0}+\frac{f_{0}\left[\tanh \left(f_{1} \tau+f_{2}\right)+1\right]}{1+g_{1} \tau+g_{2} \tau^{2}}\right]
$$

e também [85]:

$$
\chi_{2}=e^{-h_{3} / \tau-h_{4} / \tau^{2}} f_{3}\left[\tanh \left(f_{4} \tau+f_{5}\right)+1\right]
$$

onde $\tau=T / 200 \mathrm{MeV}$ e $200 \mathrm{MeV}$ é a temperatura crítica para o QGP. Os valores dos parâmetros adimensionais são [86]: $h_{0}=0.1396, h_{1}=-0.1800, h_{2}=0.0350$, $f_{0}=1.05, f_{1}=6.39, f_{2}=-4.72, g_{1}=-0.92$ e $g_{2}=0.57$. De [85] temos $h_{3}=-0.5022$, $h_{4}=0.5950, f_{3}=0.1359, f_{4}=6.3290$ e $f_{5}=-4.8303$. A pressão é calculada a partir 
de (2.99):

$$
p(T, \mu)=T^{4} \int_{0}^{T} d T^{\prime} \frac{e^{-h_{1} / \tau^{\prime}-h_{2} / \tau^{\prime 2}}}{T^{\prime}}\left[h_{0}+\frac{f_{0}\left[\tanh \left(f_{1} \tau^{\prime}+f_{2}\right)+1\right]}{1+g_{1} \tau^{\prime}+g_{2} \tau^{\prime 2}}\right]+\frac{\chi_{2}}{2} \mu^{2} T^{2}
$$

Inserindo (2.102) em (2.100) encontramos a densidade de energia:

$$
\begin{array}{r}
\varepsilon(T, \mu)=T^{4} e^{-h_{1} / \tau-h_{2} / \tau^{2}}\left[h_{0}+\frac{f_{0}\left[\tanh \left(f_{1} \tau+f_{2}\right)+1\right]}{1+g_{1} \tau+g_{2} \tau^{2}}\right]+\frac{\mu^{2}}{2} T^{3} \frac{\partial \chi_{2}}{\partial T} \\
+3 T^{4} \int_{0}^{T} d T^{\prime} \frac{e^{-h_{1} / \tau^{\prime}-h_{2} / \tau^{\prime 2}}}{T^{\prime}}\left[h_{0}+\frac{f_{0}\left[\tanh \left(f_{1} \tau^{\prime}+f_{2}\right)+1\right]}{1+g_{1} \tau^{\prime}+g_{2}{\tau^{\prime}}^{2}}\right]+\frac{3 \chi_{2}}{2} \mu^{2} T^{2}
\end{array}
$$




\section{Capítulo 3}

\section{Ondas no QGP}

\subsection{Introdução}

O plasma de quarks e glúons vem sendo produzido em laboratório no RHIC e no LHC desde os anos 2000 e assim podemos determinar suas propriedades com precisão crescente. A primeira descoberta sobre o QGP foi a de que ele é um fluido quase perfeito, com uma viscosidade muito pequena. Em se tratando de um fluido, uma das maneiras de estudar suas propriedades é observar o comportamento das ondas que se formam nele e como elas se propagam. O cálculo das propriedades das ondas no QGP ainda é um estudo em andamento, que deverá também apontar quais os efeitos mensuráveis da existência destas ondas. Podemos dizer que esta linha de pesquisa é o próximo passo no estudo do QGP.

A natureza do plasma (através da sua equação de estado) pode se manifestar no comportamento das ondas de várias maneiras e a mais evidente delas é através da velocidade do som $c_{s}^{2}=\partial p / \partial \varepsilon$. Cada equação de estado leva a uma pressão $p$ e uma densidade de energia $\varepsilon$ e consequentemente a uma velocidade do som específica.

Neste capítulo vamos discutir a conexão entre a EOS e as propriedades das ondas 
tanto lineares quanto não lineares. Vamos primeiramente nos ater ao QGP frio, que pode existir tanto em colisões nucleares a baixas energias e grande densidade bariônica, como as que serão realizadas nos experimentos FAIR e NICA, quanto no interior de estrelas compactas. Além de frio, vamos supor que o QGP seja um fluido perfeito não relativistico e assim usar as equações da hidrodinâmica não relativística. Tendo em vista principalmente as aplicações em estrelas compactas, vamos considerar que o fluido não relativístico de quarks está sujeito à ação de uma campo magnético externo constante.

Com exceção da parte com campo magnético, que é original, nos capítulos anteriores apresentamos principalmente material já conhecido na literatura. A partir de agora vamos apresentar o material original, criado por nós durante o período do doutoramento.

\subsection{Hidrodinâmica com Campo Magnético Externo}

Vamos partir das equações básicas da hidrodinâmica não relativística do fluido perfeito, que são a equação de Euler e a equação da continuidade [87]. Vamos supor que o plasma tem quarks de três sabores: $u, d$ e $s$. Mergulhados no campo magnético (sendo que qualquer campo elétrico que possa eventualmente existir, será desprezado como primeira aproximação, conforme as Refs. $[88,89]$ ), estes quarks sofrerão uma força externa de Lorentz:

$$
\vec{F}=q \vec{v} \times \vec{B}
$$

Como os quarks têm massas e cargas diferentes, a força (3.1) será diferente para cada um deles e também a aceleração. Podemos antecipar, mesmo antes de escrever as equações, que cada espécie de quark terá seu movimento próprio. As cargas positivas e negativas terão diferentes trajetórias [88]. Se a constante de acoplamento $\alpha_{s}$ (e assim a força entre os quarks) for pequena, teremos o movimento independente de três fluidos. 
Esta abordagem já foi utilizada na física de plasmas e é chamada de abordagem multifluido [90]. Seguindo esta abordagem, vamos escrever uma equação de Euler para cada quark $f=u, d, s$ :

$$
\rho_{m f}\left[\frac{\partial \overrightarrow{v_{f}}}{\partial t}+\left(\overrightarrow{v_{f}} \cdot \vec{\nabla}\right) \overrightarrow{v_{f}}\right]=-\vec{\nabla} p+\rho_{c f}\left(\overrightarrow{v_{f}} \times \vec{B}\right)
$$

onde $\rho_{m f}$ e $\rho_{c f}$ são a densidade de massa e carga de cada tipo de quark $f$. A hipótese de que a interação entre os quarks é fraca, isto é, de que a constante de acoplamento é pequena é razoável, porque já foi mostrado que esta constante diminui sob a ação de um campo magnético forte [91].

Na abordagem multi-fluido vamos supor que a pressão que cada quark experimenta é a pressão efetiva, ou seja, produzida por toda a matéria presente e não apenas pelos quarks daquela espécie. Já a velocidade, massa e carga são específicas de cada tipo.

O campo magnético uniforme e constante está orientado na direção $z$ e é dado por $\vec{B}=B \hat{z}$. A equação de continuidade para a densidade bariônica do quark de sabor $f$ é [87]:

$$
\frac{\partial \rho_{B f}}{\partial t}+\nabla \cdot\left(\rho_{B f} \overrightarrow{v_{f}}\right)=0
$$

A relação entre a densidade bariônica e a de massa é dada por $\rho_{B f}=\rho_{m f} / 3 m_{f}$. A densidade de carga é escrita como $\rho_{c u}=2 Q_{e} \rho_{B u}, \rho_{c d}=-Q_{e} \rho_{B d}$ e $\rho_{c_{s}}=-Q_{e} \rho_{B s}$.

A presença do campo magnético define uma direção privilegiada e quebra a isotropia do sistema. Assim a pressão do fluido se divive em uma pressão paralela $\left(p_{\|}\right)$e outra perpendicular $\left(p_{\perp}\right)$ à direção do campo $\vec{B}$. Consequentemente, também vamos encontrar uma velocidade do som paralela $\left(c_{s \|}\right)$ e outra perpendicular $\left(c_{s \perp}\right)$, dadas por $[92]$ :

$$
\left(c_{s \|}\right)^{2}=\frac{\partial p_{\|}}{\partial \varepsilon} \quad \text { e } \quad\left(c_{s \perp}\right)^{2}=\frac{\partial p_{\perp}}{\partial \varepsilon}
$$

Por sua vez, o gradiente de pressão será dado por:

$$
\vec{\nabla} p=\left(\frac{\partial p_{\perp}}{\partial x}, \frac{\partial p_{\perp}}{\partial y}, \frac{\partial p_{\|}}{\partial z}\right)
$$


Para estudar como o campo magnético afeta a propagação de ondas, vamos considerar o caso da mQCD com temperatura nula e campo magnético, com a definição do momento de Fermi, dada por: $k_{z, F}^{f}(n) \rightarrow k_{F}=\mu$. Assim, utilizaremos a expressão (2.74) para densidade de energia e (2.75) e (2.76) para as pressões.

Portanto, o gradiente de pressão para essa EOS é escrito na forma compacta:

$$
\vec{\nabla} p=\left(\frac{27 g^{2}}{8 m_{G}^{2}}\right) \rho_{B f} \vec{\nabla} \rho_{B f}
$$

\subsection{Ondas Lineares}

A equação de Euler é não linear. Porém quando estudamos pequenas perturbações de densidade e velocidade sobre um fundo constante, podemos fazer a aproximação linear, ou linearização [56,93-97]. Nesta aproximação dizemos, por exemplo, que a perturbação na densidade bariônica, $\delta \rho_{B}$, é muito menor do que a densidade bariônica de repouso, $\rho_{B}$, e assim conservamos apenas os termos lineares nesta perturbação. O objetivo principal ao fazer este estudo será verificar sob quais condições a onda no meio estudado (o QGP com a equação de estado mQCD) irá satisfazer as condições de causalidade e estabilidade.

\subsubsection{Linearização}

Vamos partir da equação de Euler (3.2) e da continuidade (3.3) e reescrevê-las em termos de variáveis adimensionais para a densidade:

$$
\hat{\rho}_{B f}(\vec{x}, t)=\frac{\rho_{B f}(\vec{x}, t)}{\rho_{0}}=1+\delta \rho_{B f}(\vec{x}, t)
$$

e para a velocidade

$$
\hat{\vec{v}}_{f}(\vec{x}, t)=\frac{\vec{v}_{f}(\vec{x}, t)}{c_{s}}=\delta \vec{v}_{f}(\vec{x}, t)
$$


onde $\rho_{0}$ é a densidade da configuração de equilíbrio e $c_{s}$ é a velocidade do som no meio em equilíbrio. As novas variáveis, $\delta \rho_{B_{f}}$ e $\delta \vec{v}_{f}$, são as perturbações colocadas em forma adimensional. Substituimos as expressões acima nas equações de Euler e da continuidade e, após simplificações, encontramos as seguintes equações diferenciais para as perturbações na densidade e na velocidade:

$$
3 m_{f} \rho_{0} \frac{\partial}{\partial t}\left(\delta \tilde{\vec{v}}_{f}\right)+\left(\frac{27 g^{2} \rho_{0}^{2}}{8 m_{G}^{2}}\right) \vec{\nabla}\left(\delta \rho_{B f}\right)-3 Q_{f} \rho_{0}\left(\delta \tilde{\vec{v}}_{f} \times \vec{B}\right)=0
$$

e

$$
\frac{\partial}{\partial t}\left(\delta \rho_{B f}\right)+\vec{\nabla} \cdot \delta \tilde{\vec{v}}_{f}=0
$$

onde $\delta \tilde{\vec{v}}_{f}=\left(c_{s \perp} \delta v_{f_{x}}, c_{s \perp} \delta v_{f_{y}}, c_{s \|} \delta v_{f_{z}}\right)$.

É importante notar que na derivação destas equações, que constituem a "equação de onda" do problema, desprezamos termos do tipo $\left(\delta \rho_{B}\right)^{2},\left(\delta \vec{v}_{f}\right)^{2},\left(\delta \rho_{B}\right)\left(\delta \vec{v}_{f}\right)$, ...etc., como é usual na linearização.

A solução destas equações nos dá ondas lineares de perturbações na densidade e nas componentes da velocidade. Vemos que as propriedades destas ondas, tais como, amplitude, frequência, velocidade de fase e velocidade de grupo dependem de parâmetros como as massas e cargas dos quarks, a constante de acoplamento, as velocidades do som, a massa dinâmica do glúon e o campo magnético.

$\mathrm{Na}$ próxima seção vamos nos concentrar no estudo das velocidades de fase e de grupo, que estão diretamente ligadas a condições de existência de soluções, especialmente à causalidade e à estabilidade.

\subsubsection{Causalidade e Estabilidade}

As ondas que surgem como soluções das equações diferenciais acima, só podem existir se satisfizerem dois requisitos básicos: i) causalidade, que significa que a onda não pode se propagar com uma velocidade maior do que a da luz, e ii) estabilidade, 
que significa que a amplitude (inicialmente pequena) de uma perturbação não pode crescer e tornar-se arbitrariamente grande.

Para estudar a causalidade e a estabilidade das ondas no fluido, supomos que exista uma perturbação genérica do tipo onda plana com número de onda $k$ e frequência $\omega$ que tenta atravessar o fluido. Fazemos o Ansatz [95-102]:

$$
\delta \rho_{B f}=\mathcal{D} e^{i \vec{k} \cdot \vec{x}-i \omega t}, \delta v_{f x}=\mathcal{V}_{x} e^{i \vec{k} \cdot \vec{x}-i \omega t}, \delta v_{f y}=\mathcal{V}_{y} e^{i \vec{k} \cdot \vec{x}-i \omega t} \text { e } \delta v_{f z}=\mathcal{V}_{z} e^{i \vec{k} \cdot \vec{x}-i \omega t}
$$

onde $\vec{k} \cdot \vec{x}=k_{x} x+k_{y} y+k_{z} z$, e as pequenas $(<1)$ amplitudes para as variáveis adimensionais são: $\mathcal{D}, \mathcal{V}_{x}, \mathcal{V}_{y}$ e $\mathcal{V}_{z}$. Substituindo (3.11) nas equações linearizadas (3.9) e (3.10), encontramos o sistema de equações:

$$
\begin{array}{r}
i\left(\frac{27 g_{h}^{2} \rho_{0}^{2}}{8 m_{G}^{2}}\right) k_{x} \delta \rho_{B f}-i 3 m_{f} \rho_{0} \omega\left(c_{s \perp}\right) \delta v_{f x}-3 Q_{f} \rho_{0} B\left(c_{s \perp}\right) \delta v_{f y}=0 \\
i\left(\frac{27 g_{h}^{2} \rho_{0}^{2}}{8 m_{G}^{2}}\right) k_{y} \delta \rho_{B f}+3 Q_{f} \rho_{0} B\left(c_{s \perp}\right) \delta v_{f x}-i 3 m_{f} \rho_{0} \omega\left(c_{s \perp}\right) \delta v_{f y}=0 \\
i\left(\frac{27 g_{h}^{2} \rho_{0}^{2}}{8 m_{G}^{2}}\right) k_{z} \delta \rho_{B f}-i 3 m_{f} \rho_{0} \omega\left(c_{s \|}\right) \delta v_{f z}=0 \\
-i \omega \delta \rho_{B f}+i\left(c_{s \perp}\right) k_{x} \delta v_{f x}+i\left(c_{s \perp}\right) k_{y} \delta v_{f y}+i\left(c_{s \|}\right) k_{z} \delta v_{f z}=0
\end{array}
$$

que pode ser escrito em forma matricial como:

$$
A(\omega, \vec{k}) \times\left(\begin{array}{c}
\delta \rho_{B f} \\
\delta v_{f_{x}} \\
\delta v_{f_{y}} \\
\delta v_{f_{z}}
\end{array}\right)=0
$$


onde $A(\omega, \vec{k})$ é a matriz:

$$
A(\omega, \vec{k})=\left(\begin{array}{cccc}
i\left(\frac{27 g_{h}{ }^{2} \rho_{0}{ }^{2}}{8 m_{G}{ }^{2}}\right) k_{x} & -i 3 m_{f} \rho_{0} \omega\left(c_{s \perp}\right) & -3 Q_{f} \rho_{0} B\left(c_{s \perp}\right) & 0 \\
i\left(\frac{27 g_{h}{ }^{2} \rho_{0}{ }^{2}}{8 m_{G}{ }^{2}}\right) k_{y} & 3 Q_{f} \rho_{0} B\left(c_{s \perp}\right) & -i 3 m_{f} \rho_{0} \omega\left(c_{s \perp}\right) & 0 \\
i\left(\frac{27 g_{h}{ }^{2} \rho_{0}{ }^{2}}{8 m_{G}{ }^{2}}\right) k_{z} & 0 & 0 & -i 3 m_{f} \rho_{0} \omega\left(c_{s \|}\right) \\
-i \omega & i\left(c_{s \perp}\right) k_{x} & i\left(c_{s \perp}\right) k_{y} & i\left(c_{s \|}\right) k_{z}
\end{array}\right)
$$

A relação de dispersão é encontrada impondo a condição de existência de soluções não triviais:

$$
\operatorname{det} A(\omega, \vec{k})=0
$$

e resolvendo a equação resultante para $\omega$, encontramos a chamada relação de dispersão, uma equação que relaciona $\vec{k}$ e $\omega$. A frequência $\omega$ é escrita como [95, 97, 100-102]:

$$
\omega=\omega_{R}+i \omega_{I}
$$

onde as partes real e imaginária satifazem $\omega_{R} \in \mathbb{R}$ e $\omega_{I} \in \mathbb{R}$. A causalidade é garantida quando as seguintes condições para $\omega_{R}$ e $\omega_{I}$ forem satisfeitas [103]:

$$
\lim _{|\vec{k}| \rightarrow \infty}\left|\frac{\omega_{R}}{|\vec{k}|}\right|<1
$$

e

$$
\lim _{|\vec{k}| \rightarrow \infty}\left|\frac{\omega_{I}}{|\vec{k}|}\right|<\infty
$$

A condição (3.15) indica que a velocidade de fase, $\left|\overrightarrow{v_{p}}\right|$, é menor que a unidade (a velocidade da luz em unidades naturais), ou seja, $\left|\overrightarrow{v_{p}}\right|<1$, onde [100-102]:

$$
\overrightarrow{v_{p}}=\frac{\omega_{R}}{|\vec{k}|} \hat{k}=\frac{\omega_{R}}{|\vec{k}|^{2}} \vec{k}
$$


Além da condição acima, temos que garantir que a velocidade de grupo, $v_{g}$, dada por [101-103]:

$$
\overrightarrow{v_{g}}=\left(\frac{\partial \omega_{R}}{\partial k_{x}}, \frac{\partial \omega_{R}}{\partial k_{y}}, \frac{\partial \omega_{R}}{\partial k_{z}}\right)
$$

seja finita quando $k \rightarrow \infty$.

A estabilidade é garantida quando $\omega_{I}<0$, pois $e^{i \vec{k} \cdot \vec{x}-i \omega t}=e^{\omega_{I} t} e^{i \vec{k} \cdot \vec{x}-i \omega_{R} t}$ e $e^{\omega_{I} t}$ devem ser decrescentes como função do tempo.

Calculando $\operatorname{det} A(\omega, \vec{k})=0$ para $\omega$, obtemos:

$$
\omega^{4}-\left[\left(\tilde{c}_{s}\right)^{2}\left(k_{x}{ }^{2}+k_{y}{ }^{2}+k_{z}{ }^{2}\right)+\left(\frac{B^{2} Q_{f}^{2}}{m_{f}^{2}}\right)\right] \omega^{2}+\left(\frac{B^{2} Q_{f}^{2}}{m_{f}^{2}}\right)\left(\tilde{c}_{s}\right)^{2} k_{z}{ }^{2}=0
$$

sendo $\tilde{c}_{s}$ a "velocidade do som efetiva":

$$
\left(\tilde{c}_{s}\right)^{2} \equiv \frac{9 g^{2} \rho_{0}}{8 m_{f} m_{G}^{2}}
$$

que depende das propriedades da equação de estado. As soluções da equação acima são dadas por:

$$
\omega_{ \pm}^{2}=\frac{\left(\tilde{c}_{s}\right)^{2}}{2}|\vec{k}|^{2}+\frac{B^{2} Q_{f}^{2}}{2 m_{f}^{2}} \pm \sqrt{\frac{1}{4}\left(\left(\tilde{c}_{s}\right)^{2}|\vec{k}|^{2}+\frac{B^{2} Q_{f}^{2}}{m_{f}^{2}}\right)^{2}-\frac{B^{2} Q_{f}^{2}}{m_{f}^{2}}\left(\tilde{c}_{s}\right)^{2} k_{z}^{2}}
$$

As quatro soluções de $(3.21)$ são $\omega(\vec{k})= \pm \sqrt{\omega^{2}}$. Notamos que $\omega(\vec{k}) \in \mathbb{R}$ e $\omega_{I}=0$, o que garante a estabilidade.

Assim, a velocidade de fase é:

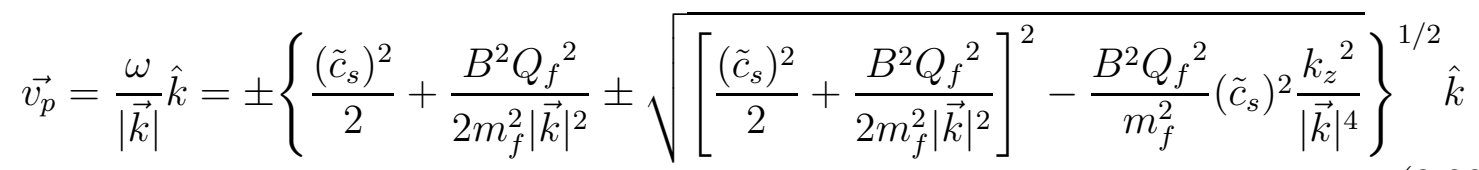


que no limite (3.15) fornece:

$$
\lim _{|\vec{k}| \rightarrow \infty}\left|\frac{\omega}{|\vec{k}|}\right| \cong \lim _{|\vec{k}| \rightarrow \infty} \sqrt{\frac{\left(\tilde{c}_{s}\right)^{2}}{2} \pm \frac{\left(\tilde{c}_{s}\right)^{2}}{2}}=\tilde{c}_{s} \text { ou } 0
$$

Portanto, vemos que a causalidade é satisfeita.

As componentes da velocidade de grupo (3.18) são:

$$
\frac{\partial \omega}{\partial k_{x, y}}= \pm \frac{1}{2 \omega}\left\{\left(\tilde{c}_{s}\right)^{2} k_{x, y} \pm \frac{\left[\left(\tilde{c}_{s}\right)^{2}|\vec{k}|^{2}+\frac{B^{2} Q_{f}^{2}}{m_{f}^{2}}\right]\left(\tilde{c}_{s}\right)^{2} k_{x, y}}{2 \sqrt{\left(\tilde{c}_{s}\right)^{2} \frac{|\vec{k}|^{2}}{2}+\frac{B^{2} Q_{f}^{2}}{2 m_{f}^{2}}-\frac{B^{2} Q_{f}^{2}}{m_{f}^{2}}\left(\tilde{c}_{s}\right)^{2} k_{z}^{2}}}\right\}
$$

e

$$
\frac{\partial \omega}{\partial k_{z}}= \pm \frac{1}{2 \omega}\left\{\left(\tilde{c}_{s}\right)^{2} k_{z} \pm \frac{\left[\left(\tilde{c}_{s}\right)^{2}|\vec{k}|^{2}-\frac{B^{2} Q_{f}^{2}}{m_{f}^{2}}\right]\left(\tilde{c}_{s}\right)^{2} k_{z}}{2 \sqrt{\left(\tilde{c}_{s}\right)^{2} \frac{|\vec{k}|^{2}}{2}+\frac{B^{2} Q_{f}^{2}}{2 m_{f}^{2}}-\frac{B^{2} Q_{f}^{2}}{m_{f}^{2}}\left(\tilde{c}_{s}\right)^{2} k_{z}^{2}}}\right\}
$$

As equações acima mostram que $\left|\overrightarrow{v_{g}}\right|<\infty$ com o aumento do número de onda, o que também é necessário para que a causalidade seja satisfeita.

Vamos agora considerar alguns casos especiais. Quando o campo magnético se anula, $B=0$, temos:

$$
\omega(\vec{k})= \pm\left(\tilde{c}_{s}\right)|\vec{k}|
$$

$\mathrm{e}$

$$
\left|\overrightarrow{v_{p}}\right|=\tilde{c}_{s}
$$

e assim recuperamos o limite esperado, encontrado em [96]. Quando o campo magnético é muito intenso, $\left|Q_{f}\right| B>\mu^{2}>m_{f}^{2}$, a relação de dispersão toma a forma:

$$
\omega^{4}-\left[\left(\tilde{c}_{s}\right)^{2}|\vec{k}|^{2}+\left(\mathcal{V}_{s}\right)^{2} k_{z}{ }^{2}+\frac{B^{2} Q_{f}{ }^{2}}{m_{f}^{2}}\right] \omega^{2}+\frac{B^{2} Q_{f}{ }^{2}}{m_{f}^{2}}\left(\mathcal{V}_{s}\right)^{2} k_{z}{ }^{2}+\frac{B^{2} Q_{f}{ }^{2}}{m_{f}^{2}}\left(\tilde{c}_{s}\right)^{2} k_{z}{ }^{2}=0
$$


onde $\left(\mathcal{V}_{s}\right)^{2}$ é dado por:

$$
\left(\mathcal{V}_{s}\right)^{2}=\frac{\tilde{Q} \mu}{\left|Q_{f}\right| m_{f}}-\frac{\left|Q_{f}\right| m_{f}}{2 \tilde{Q} \mu}
$$

e $\tilde{Q} \equiv \sum_{j=u}^{d, s}\left|Q_{f}\right|$. Desta equação podemos obter $\omega$ e tomar o limite de grandes números de onda $k$, encontrando:

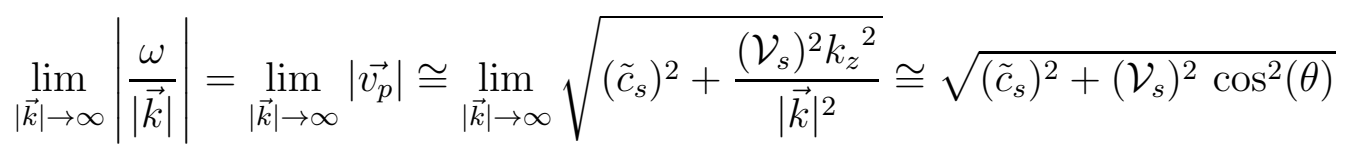

onde $\theta$ é o ângulo entre o vetor $\vec{k}$ e a direção $z$. Como $\mathcal{V}_{s}$ é sempre maior do que um, a causalidade vale apenas em certas direções de propagação. Quando ela se dá na direção do campo magnético $\left(\theta=0\right.$ e $\left.k_{z}=|\vec{k}|\right)$, encontramos $\left|\overrightarrow{v_{p}}\right|>1$, que não é um resultado fisicamente aceitável. Em geral, isto quer dizer que levamos o formalismo (não relativístico) para além de sua validade, ou que fizemos alguma aproximação que estava em conflito com outra feita anteriormente. Porém, ainda não podemos descartar a possibilidade de ter encontrado alguma instabilidade física, pois elas já foram observadas em outros contextos em que havia um campo magnético muito forte.

O estudo realizado nesta seção visava determinar os efeitos de um campo magnético constante na propagação de ondas na matéria de quarks fria e não relativística. Para isto foram adicionados termos contendo este campo na equação de estado e nas equações de movimento. Levamos em conta o termo da força de Lorentz e obtivemos a relação de dispersão para perturbações na densidade e na velocidade. Os efeitos de anisotropia causados pelo campo $B$ são vistos, principalmente, na velocidade do som, que passa a ter uma componente paralela e outra perpendicular ao campo magnético. Vimos que a introdução deste campo não gera instabilidades nas ondas, porém em algumas situações a velocidade de fase pode ser maior do que um. Isto pode ocorrer para ondas que se movimentam na direção paralela ao campo, quando este possui uma intensidade muito alta.

Quando as perturbações não são suficientemente pequenas, não podemos usar as expansões descritas nesta seção e truncá-las após o primeiro termo. Teríamos que 
considerar potências mais altas de $\delta \rho_{B}$ e $\delta \vec{v}$. Isto, por sua vez nos levaria a equações diferenciais não lineares, o que será discutido na próxima seção.

\subsection{Ondas Não Lineares}

A extensão das expansões feitas anteriormente para além da linearização nos leva não apenas a um cálculo mais preciso (por considerar mais termos de uma série), mas a resultados qualitativamente novos e surpreendentes. O mais famoso destes resultados é o sóliton de Korteweg-de-Vries (KdV) [104-106]. Trata-se de uma onda solitária que se propaga por grandes distâncias sem mudar a sua forma. Ela resulta da combinação entre o termo não linear $(v \partial v / \partial x)$ e um termo dispersivo $\left(\partial^{3} v / \partial x^{3}\right)$ que surge, por exemplo por causa do uso de certas equações de estado. Neste caso, as perturbações que se propagam pelo fluido, incluem efeitos de termos de ordem mais alta em $\delta$, como veremos nas próximas seções.

\subsubsection{Método das Perturbações Redutivas (RPM)}

Um método bastante usado para estudar perturbações em sistemas governados por equações não lineares, como a equação de Euler, é o Método das Perturbações Redutivas (ou Reductive Perturbation Method, RPM) [88,107-116]. Com este método fazemos simultaneamente uma expansão em série das grandezas físicas relevantes (por exemplo a densidade) e uma transformação das coordenadas. A expansão e a transformação de coordenadas estão conectadas. Ambas possuem o parâmetro pequeno (às vezes chamado de $\sigma$ ou de $\epsilon$ ) que aparece nas expansões em série. Ao realizar estas duas operações, ocorrem cancelamentos não triviais e o resultado são equações diferenciais não lineares para as perturbações.

Vamos aplicar o RPM nas equações da hidrodinâmica, (3.2) e (3.3), para obter equações de onda não lineares que regem as perturbações na densidade bariônica e na 
velocidade. Como foi mencionado, esta técnica vai além da linearização e ao mesmo tempo preserva os termos não lineares nas equações de onda.

Definimos uma densidade de fundo, $\rho_{0}$, que normalmente é dada em termos da densidade da matéria nuclear em equilíbrio: $\rho_{N}=0.17 \mathrm{fm}^{-3}$. A seguir, no RPM fazemos uma mudança de variáveis $(x, y, z, t) \rightarrow(X, Y, Z, T)$, usando as coordenadas "esticadas" ou "stretched" [107-111,116]:

$$
\begin{gathered}
X=\sigma^{1 / 2}\left(x-c_{s \perp} t\right) \\
Y=\sigma y \\
Z=\sigma z \\
T=\sigma^{3 / 2} t
\end{gathered}
$$

Finalmente, seguindo o procedimento algébrico descrito em [112-114,116], fazendo a seguinte transformação para o campo magnético:

$$
B=\sigma \tilde{B}
$$

vamos encontrar equações no espaço $(X, Y, Z, T)$ contendo o parâmetro pequeno $\sigma$, que é o parâmetro da expansão na densidade e nas velocidades adimensionais:

$$
\begin{aligned}
& \hat{\rho}_{B f}(x, y, z, t)=\frac{\rho_{B f}}{\rho_{0}}=1+\sigma \rho_{f_{1}}(x, y, z, t)+\sigma^{2} \rho_{f_{2}}(x, y, z, t)+\sigma^{3} \rho_{f_{3}}(x, y, z, t)+\ldots \\
& \hat{v}_{f x}(x, y, z, t)=\frac{v_{f x}}{c_{s \perp}}=\sigma v_{f x_{1}}(x, y, z, t)+\sigma^{2} v_{f x_{2}}(x, y, z, t)+\sigma^{3} v_{f x_{3}}(x, y, z, t)+\ldots \\
& \hat{v}_{f y}(x, y, z, t)=\frac{v_{f y}}{c_{s \perp}}=\sigma^{3 / 2} v_{f y_{1}}(x, y, z, t)+\sigma^{2} v_{f y_{2}}(x, y, z, t)+\sigma^{5 / 2} v_{f y_{3}}(x, y, z, t)+\ldots \\
& \hat{v}_{f z}(x, y, z, t)=\frac{v_{f z}}{c_{s \|}}=\sigma^{3 / 2} v_{f z_{1}}(x, y, z, t)+\sigma^{2} v_{f z_{2}}(x, y, z, t)+\sigma^{5 / 2} v_{f z_{3}}(x, y, z, t)+\ldots
\end{aligned}
$$

Substituindo as equações acima em (3.2) e (3.3), desprezando os termos de ordem 
$n>2$ em $\sigma^{n}$ e agrupando os termos $\sigma, \sigma^{3 / 2}$ e $\sigma^{2}$, chegamos a:

$$
\begin{gathered}
\sigma\left\{-\frac{\partial \rho_{f_{1}}}{\partial X}+\frac{\partial v_{f x_{1}}}{\partial X}\right\}+\sigma^{2}\left\{-\frac{\partial \rho_{f_{2}}}{\partial X}+\frac{\partial v_{f x_{2}}}{\partial X}+\frac{1}{\left(c_{s \perp}\right)} \frac{\partial \rho_{f_{1}}}{\partial T}+\rho_{f_{1}} \frac{\partial v_{f x_{1}}}{\partial X}+v_{f x_{1}} \frac{\partial \rho_{f_{1}}}{\partial X}\right. \\
\left.+\frac{\partial v_{f y_{1}}}{\partial Y}+\left(\frac{c_{s} \|}{c_{s \perp}}\right) \frac{\partial v_{f z_{1}}}{\partial Z}\right\}=0
\end{gathered}
$$

para a equação da continuidade. No caso da equação de Euler, usando o gradiente (3.6) encontramos:

$$
\begin{gathered}
\sigma\left\{-\frac{\partial v_{f x_{1}}}{\partial X}+\left(\frac{9 g^{2} \rho_{0}}{8 m_{f} m_{G}^{2}\left(c_{s \perp}\right)^{2}}\right) \frac{\partial \rho_{f_{1}}}{\partial X}\right\}+\sigma^{2}\left\{-\frac{\partial v_{f x_{2}}}{\partial X}+\frac{1}{\left(c_{s \perp}\right)} \frac{\partial v_{f x_{1}}}{\partial T}+v_{f x_{1}} \frac{\partial v_{f x_{1}}}{\partial X}\right. \\
\left.-\rho_{f_{1}} \frac{\partial v_{f x_{1}}}{\partial X}+\rho_{f_{1}} \frac{\partial \rho_{f_{1}}}{\partial X}+\left(\frac{9 g^{2} \rho_{0}}{8 m_{f} m_{G}^{2}\left(c_{s \perp}\right)^{2}}\right) \frac{\partial \rho_{f_{2}}}{\partial X}-\frac{Q_{f} \tilde{B}}{m_{f}\left(c_{s \perp}\right)} v_{f y_{1}}\right\}=0 \\
\sigma^{3 / 2}\left\{-\frac{\partial v_{f y_{1}}}{\partial X}+\left(\frac{9 g^{2} \rho_{0}}{8 m_{f} m_{G}^{2}\left(c_{s \perp}\right)^{2}}\right) \frac{\partial \rho_{f 1}}{\partial Y}+\frac{Q_{f} \tilde{B}}{m_{f}\left(c_{s \perp}\right)} v_{f x_{1}}\right\}+\sigma^{2}\left\{-\frac{\partial v_{f y_{2}}}{\partial X}\right\}=0 \\
\sigma^{3 / 2}\left\{-\left(\frac{c_{s \|}}{c_{s \perp}}\right) \frac{\partial v_{f z_{1}}}{\partial X}+\left(\frac{9 g^{2} \rho_{0}}{8 m_{f} m_{G}^{2}\left(c_{s \perp}\right)^{2}}\right) \frac{\partial \rho_{f 1}}{\partial Z}\right\}+\sigma^{2}\left\{-\left(\frac{c_{s \|}}{c_{s \perp}}\right) \frac{\partial v_{f z_{2}}}{\partial X}\right\}=0
\end{gathered}
$$

Resolvendo este sistema de equações, para cada ordem de $\sigma$, obtemos:

$$
\frac{\partial}{\partial X}\left[\frac{\partial \rho_{f_{1}}}{\partial T}+\frac{3}{2}\left(c_{s \perp}\right) \rho_{f_{1}} \frac{\partial \rho_{f_{1}}}{\partial X}\right]+\frac{\left(c_{s \perp}\right)}{2}\left(\frac{\partial^{2} \rho_{f_{1}}}{\partial Y^{2}}+\frac{\partial^{2} \rho_{f_{1}}}{\partial Z^{2}}\right)=\frac{\left(Q_{f} \tilde{B}\right)^{2}}{2 m_{f}^{2}\left(c_{s \perp}\right)} \rho_{f_{1}}
$$

Dos termos de $\mathcal{O}(\sigma)$, surge a seguinte condição que deve ser satisfeita pela velocidade do som perpendicular:

$$
\left(c_{s \perp}\right)^{2}=\frac{9 g^{2} \rho_{0}}{8 m_{f} m_{G}^{2}}
$$

que coincide com a "velocidade do som efetiva" encontrada em (3.20). Reescrevendo 
a equação (3.37) nas coordenadas cartesianas originais, temos:

$$
\begin{aligned}
& \frac{\partial}{\partial x}\left[\frac{\partial}{\partial t} \delta \rho_{B f}+\left(c_{s \perp}\right) \frac{\partial}{\partial x} \delta \rho_{B f}+\frac{3}{2}\left(c_{s \perp}\right) \delta \rho_{B f} \frac{\partial}{\partial x} \delta \rho_{B f}\right] \\
& +\frac{\left(c_{s \perp}\right)}{2}\left(\frac{\partial^{2}}{\partial y^{2}} \delta \rho_{B f}+\frac{\partial^{2}}{\partial z^{2}} \delta \rho_{B f}\right)=\frac{\left(Q_{f} B\right)^{2}}{2 m_{f}^{2}\left(c_{s \perp}\right)} \delta \rho_{B f}
\end{aligned}
$$

onde $\delta \rho_{B f} \equiv \sigma \rho_{f_{1}}$ é a perturbação na densidade bariônica sobre o valor de equilíbrio $\rho_{0}$, como pode ser visto em (3.29). A equação acima pode ser escrita de uma forma mais compacta se fizermos mais uma transformação de variáveis e, seguindo [116], introduzirmos a variável $\xi=x+y+z$. Vamos encontrar:

$$
\frac{\partial}{\partial \xi}\left[\frac{\partial}{\partial t} \delta \rho_{B f}+2\left(c_{s \perp}\right) \frac{\partial}{\partial \xi} \delta \rho_{B f}+\frac{3}{2}\left(c_{s \perp}\right) \delta \rho_{B f} \frac{\partial}{\partial \xi} \delta \rho_{B f}\right]=\frac{\left(Q_{f} B\right)^{2}}{2 m_{f}^{2}\left(c_{s \perp}\right)} \delta \rho_{B f}
$$

\subsubsection{Equação Reduzida de Ostrovsky}

A equação (3.40) é uma espécie de "equação de quebra de onda inomogênea em três dimensões", que pode ser reescrita em uma forma mais compacta:

$$
\frac{\partial}{\partial \xi}\left[\frac{\partial}{\partial t} \delta \rho_{B f}+\alpha \frac{\partial}{\partial \xi} \delta \rho_{B f}+\beta \delta \rho_{B f} \frac{\partial}{\partial \xi} \delta \rho_{B f}\right]=\Gamma \delta \rho_{B f}
$$

onde $\beta$ é o coeficiente do termo não linear, $\alpha$ é a velocidade da onda linear sem dispersão e $\Gamma$ o coeficiente de dispersão, dado por:

$$
\Gamma=\frac{\left(Q_{f} B\right)^{2}}{2 m_{f}^{2}\left(c_{s \perp}\right)}
$$

Caso o campo magnético fosse nulo, a equação acima seria convertida em uma equação de quebra de onda. Assim podemos dizer que a presença de um campo magnético permite a existência de soluções solitônicas. A equação (3.41) é conhecida na literatura como Equação Reduzida de Ostrovsky (Reduced Ostrovisky Equation, 
ou ROE) ou ainda equação Ostrovsky-Hunter (Ostrovsky-Hunter Equation, ou OHE) quando $\Gamma>0$ [117-124], que é o caso estudado.

A ROE é um caso particular da equação de Ostrovsky [125], para uma função geral $f(\xi, t)$ :

$$
\frac{\partial}{\partial \xi}\left[\frac{\partial}{\partial t} f+\alpha \frac{\partial}{\partial \xi} f+\beta f \frac{\partial}{\partial \xi} f+\Pi \frac{\partial^{3}}{\partial \xi^{3}} f\right]=\Gamma f
$$

quando o coeficiente de dispersão de alta frequência ПI é nulo. Esta equação descreve ondas internas e superfícies fracamente não lineares em um oceano em rotação [125].

A solução da ROE se encontra no Apêndice D, e é dada por:

$$
\delta \rho_{B f}(\xi, t)=-\frac{6 \gamma^{2} \lambda^{2}}{\beta \Gamma} \operatorname{sech}^{2}[\lambda(\Omega-\gamma t)]
$$

onde $\lambda$ e $\gamma$ são constantes de integração e $\gamma$ está relacionada com a velocidade de propagação da perturbação. O sinal negativo indica que a variação da densidade com respeito a $\rho_{0}$ é negativa, ou seja, que a perturbação é um pulso de rarefação. A nova variável $\Omega$ está relacionada com as coordenadas cartesianas através da equação:

$$
\xi=x+y+z=\Omega+\alpha t+\xi_{0}+\frac{6 \gamma \lambda}{\beta \Gamma}\{\tanh [\lambda(\Omega-\gamma t)]-1\}
$$

Para um dado valor da coordenada $\xi$ é possível resolver a equação acima e encontrar $\Omega$ que é substituído em (3.44), que representa um pulso que se move para direita presevando a sua forma.

Pela solução da ROE, vemos que a amplitude da onda de densidade é proporcional à $1 / \Gamma$ e assim, com o aumento de $B$ a amplitude da onda diminui. Vemos também que ondas da densidade de quarks mais massivos têm amplitude maior.

Para ilustrar o comportamento de sóliton (3.44) mostramos na Figura 3.1 a perturbação $\left|\delta \rho_{B f}\right|$ como função de $x$ para valores fixos de $y=0$ e $z=0$ e para dois valores do tempo t. A perturbação é na densidade do quark up para três valores do campo magnético, que foram escolhidos para satisfazer $0<\left|\delta \rho_{B u}\right|<1$ e respeitar (3.29), já que $\delta \rho_{B u} \equiv \sigma \rho_{u 1}$. Para campos magnéticos $\sim 10^{16} G$ ou menores, temos 
que $\left|\delta \rho_{B u}\right|>1$. Os parâmetros utilizados são: $B_{Q C D}=70 \mathrm{MeV} / \mathrm{fm}^{3}, g=0.05$, $m_{G}=600 \mathrm{MeV}, \xi_{0}=20 \mathrm{fm}, \lambda=1 \mathrm{fm}^{-1}$ e $\gamma=0.1$. O potencial químico utilizado é $\nu_{f}=300 \mathrm{MeV}$. Para os valores escolhidos do campo magnético as densidades de fundo são $\rho_{0}=2 \rho_{N} \sim 2.1 \rho_{N}\left(B=10^{17} G \sim 10^{19} G\right)$, que implicam $c_{s} \cong 0.2$. A velocidade de propagação do pulso é $\alpha+\gamma=0.5$ e não viola a causalidade.

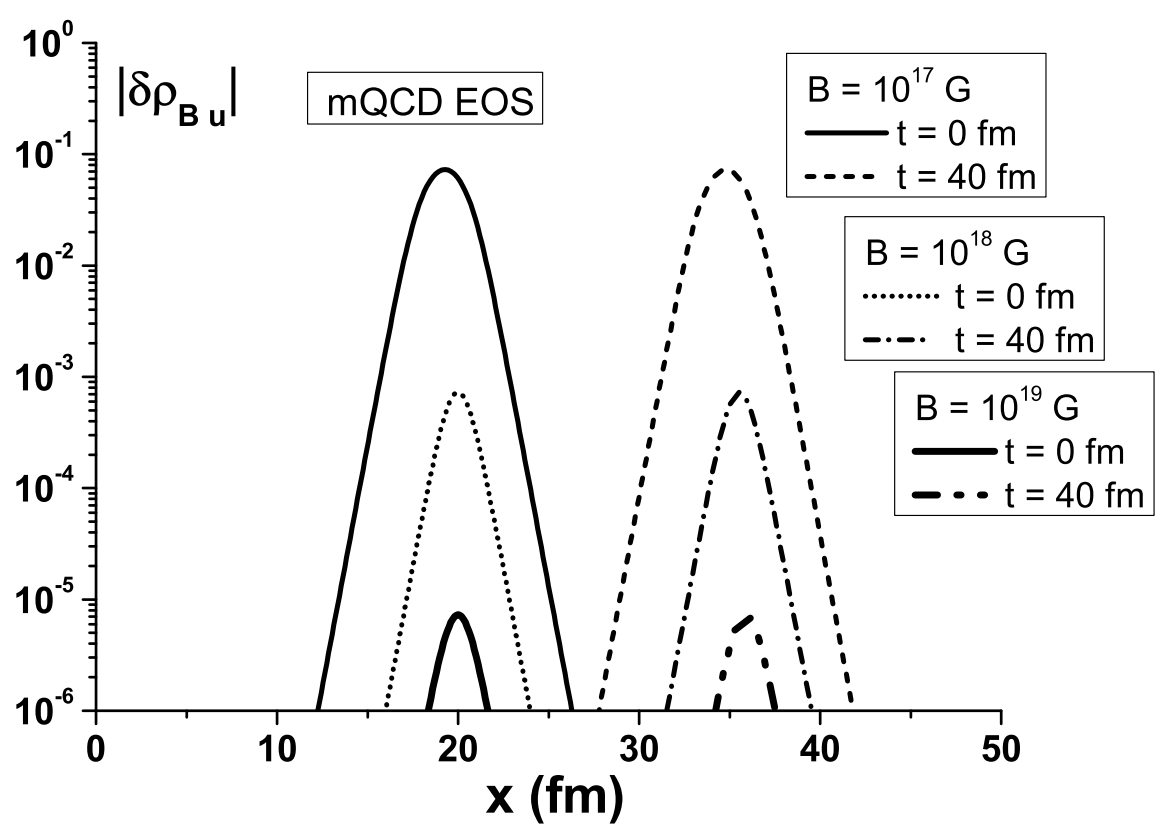

Figura 3.1: Comportamento solitônico da perturbação na densidade bariônica, $\left|\delta \rho_{B u}\right|$, para diversos tempos e intensidade de campo magnético. Usamos a equação de estado na aproximação de campo médio (mQCD)

Nesta seção estudamos a propagação de ondas não lineares na matéria de quarks fria e magnetizada. Incluímos o campo magnético na equação de estado e nas equações da hidrodinâmica. Derivamos uma equação de onda para a perturbação na densidade bariônica, que foi identificada como uma equação reduzida de Ostrovsky. A ROE possui uma solução analítica que é dada por um pulso de rarefação do tipo sóliton na perturbação bariônica. Em um nível qualitativo observamos que o campo magnético, que entra no coeficiente $\Gamma$, reduz a amplitude da onda. 


\subsection{Conclusão}

Neste capítulo estudamos a propagação de ondas no plasma de quarks e glúons frio com um campo magnético. Utilizamos a equação de estado chamada de mQCD. Na primeira parte fizemos um estudo com ondas lineares. Linearizamos as equações da hidrodinâmica e juntamente com o gradiente de pressão obtido com a mQCD, mostramos que a introdução do campo magnético não causa instabilidade na propagação deste tipo de onda. Mostramos que a mQCD é uma teoria que respeita a causalidade. Na segunda parte estudamos as ondas não lineares. Utilizamos o RPM para preservar os termos não lineares das equações de onda e obtivemos como resultado a ROE, que possui uma solução analítica: um pulso de rarefação do tipo sóliton. Estes resultados podem ser relevantes para estudar propagações de ondas no interior de estrelas compactas. Como o sóliton se propaga sem se deformar ele poderia caminhar metros ou até quilômetros. Trata-se de um fenômeno inicialmente microscópico que pode ter efeitos macroscópicos. 


\section{Capítulo 4}

\section{Aplicações}

\subsection{Estrela de Quarks com Campo Magnético}

Uma estrela de nêutrons é um dos possíveis estágios finais para os quais uma estrela evolui. Nestes objetos, caso a densidade seja alta o suficiente, os nucleons podem sofrer uma transição de fase para o QGP. Também é possível que a estrela seja constituída inteiramente de quarks desconfinados. Estas são as chamadas estrelas de quarks [126]. Estes objetos são portanto interessantes para aprendermos mais sobre QCD. Especialmente agora, depois da descoberta de ondas gravitacionais, que trazem novas informações sobre as estrelas compactas.

\subsubsection{Propriedades das Estrelas de Nêutrons}

Estes objetos, que têm raio de algumas dezenas de quilômetros e massas da ordem da massa solar, atingem densidades bastante altas, $\rho \sim 10^{15} \mathrm{~g} / \mathrm{cm}^{3}$ e acredita-se que possuam campos magnéticos da ordem de $10^{18} \mathrm{G}$. A uma estrela de nêutrons com 
campo magnético damos o nome de pulsar. Um pulsar possui uma alta taxa de rotação (com período da ordem de $\sim 1 m s$ ) e seu eixo de rotação não está alinhado com o eixo do campo magnético. Temos a impressão de que ele está pulsando quando o feixe eletromagnético passa pelo eixo de visão do observador, como pode ser visto na Figura 4.1 .
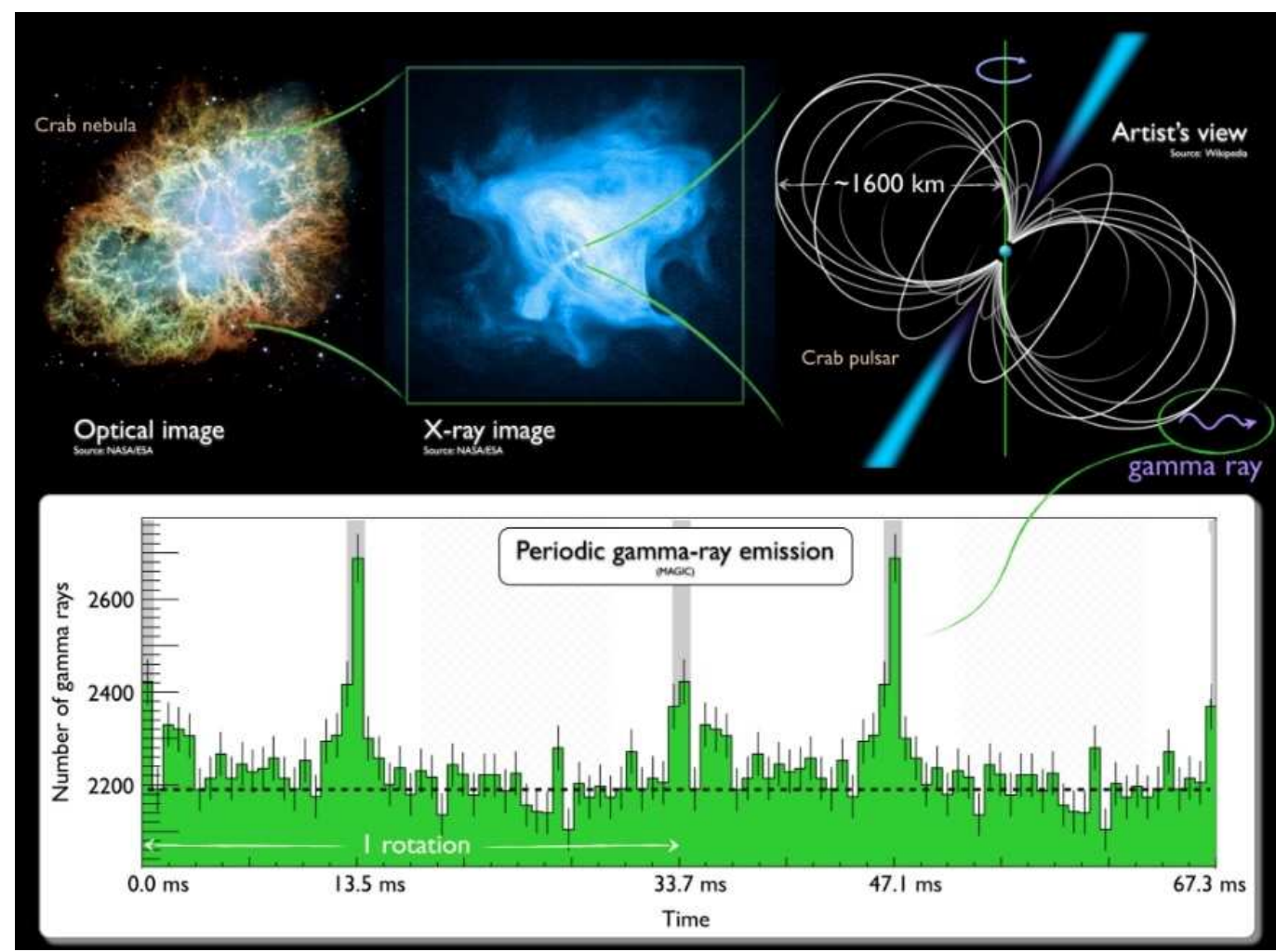

Figura 4.1: Imagens em ótico, raios-X e representação gráfica de um pulsar, abaixo se tem a emissão regular de raios gamma com período de $0.0337 \mathrm{~s}$ [127].

Uma estrela compacta é um objeto com carga nula e portanto os quarks e elétrons que o constituem devem estar em equilíbrio de carga:

$$
\frac{2}{3} \rho_{u}=\frac{1}{3} \rho_{d}+\frac{1}{3} \rho_{s}+\rho_{e}
$$


A densidade bariônica é dada por:

$$
\rho_{B}=\frac{1}{3} \rho=\frac{1}{3}\left(\rho_{u}+\rho_{d}+\rho_{s}\right)
$$

Vamos sempre estudar sistemas em equilíbrio químico. Isto significa que as multiplicidades de todas as partículas são constantes no tempo. Assim as reações mediadas pelas interações fracas, que criam e destroem quarks, elétrons, neutrinos e as antipartículas correspondentes, devem estar em equilíbrio [126,128]:

$$
\begin{gathered}
u+e^{-} \rightarrow d+\nu_{e}, \quad u+e^{-} \rightarrow s+\nu_{e}, \\
d \rightarrow u+e^{-}+\bar{\nu}_{e}, \quad s \rightarrow u+e^{-}+\bar{\nu}_{e}, \quad \text { e } \quad s+u \rightarrow d+u .
\end{gathered}
$$

A existência de equilíbrio químico impõe relações entre os potenciais químicos dos quarks $u, d$ e $s$ e elétrons:

$$
\nu_{d}=\nu_{s} \equiv \mu \quad \text { e } \quad \nu_{u}+\mu_{e}=\mu
$$

Para que um sistema de quarks permaneça desconfinado, a energia dividida pelo número de bárions não pode ser muito baixa (ou os quarks voltariam a estar confinados em hádrons) e não pode ser muito alta para que o sistema não decaia em novas partículas. Assim, esta razão para um sistema somente de quarks $u$ e $d$ não deve ser menor do que a menor razão encontrada no núcleo, que é de $930 \mathrm{MeV}$ para o ferro [128]. Quando existem também quarks estranhos, s, este valor é de $934 \mathrm{MeV}$ [34]. Esta condição de não decaimento (ou de estabilidade) pode ser escrita como:

$$
\left.\frac{\varepsilon}{\rho_{B}}\right|_{(3 \text {-sabores })} \leq 934 \mathrm{MeV} \leq\left.\frac{\varepsilon}{\rho_{B}}\right|_{(2 \text {-sabores })}
$$




\subsubsection{Massa e Raio}

Há quase oitenta anos, utilizando a equação de estado do gás de nêutrons, Oppenheimer e Volkoff fizeram a primeira estimativa da massa de uma estrela de nêutrons e encontraram $0.7 M_{\odot}[129]$, valor que ficou conhecido como limite de OppenheimerVolkoff. Desde então surgiram muitas medidas e cálculos teóricos. Um dos resultados experimentais mais recentes foi a medida da massa do pulsar PSR J1614-2230, que tem a massa igual a $(1.97 \pm 0.04) M_{\odot}[130]$. Mais tarde um outro grupo experimental mediu a massa do pulsar PSR B1957+20, igual a $(2.4 \pm 0.12) M_{\odot}[131]$. Finalmente, foi medida a massa do pulsar PSR J0348+0432, igual a $(2.01 \pm 0.04) M_{\odot}$ [132]. Do ponto de vista teórico, a massa e o raio dependem principalmente da equação de estado que se utiliza. Na Figura 4.2 mostramos o assim chamado diagrama massa-raio obtido com diversas EOS.

As linhas retas estabelecem condições que devem ser satisfeitas. Por exemplo, as equações de estado que dêem origem a linhas que se aproximem demais do canto esquerdo superior e cruzem a linha "causality" serão eliminadas por violarem a causalidade. As linhas horizontais, estabelecem massas experimentais que devem ser atingidas pelas previsões das diversas equações de estado. Assim vemos, por exemplo, que as EOS SQM1 e PAL6 estão excluidas por não atingirem a linha horizontal correspondente a 2 massas solares. As linhas que sobem a partir do canto esquerdo inferior são obtidas a partir de equações de estado que representam matéria feita somente de quarks. As linhas que sobem a partir do canto direito inferior são obtidas a partir de equações de estado que representam matéria feita somente de bárions e mésons. De um modo geral vemos que os modelos hadrônicos atingem massas maiores do que os modelos de quarks. Isto decorre do fato de que as EOS dos modelos de quarks são mais "soft" (suaves ou moles), ou seja, geram menos pressão. Uma das razões que nos levam a utilizar a mQCD é que ela é capaz de gerar pressões maiores.

Para construir o diagrama massa-raio temos que resolver as equações de Tolman- 


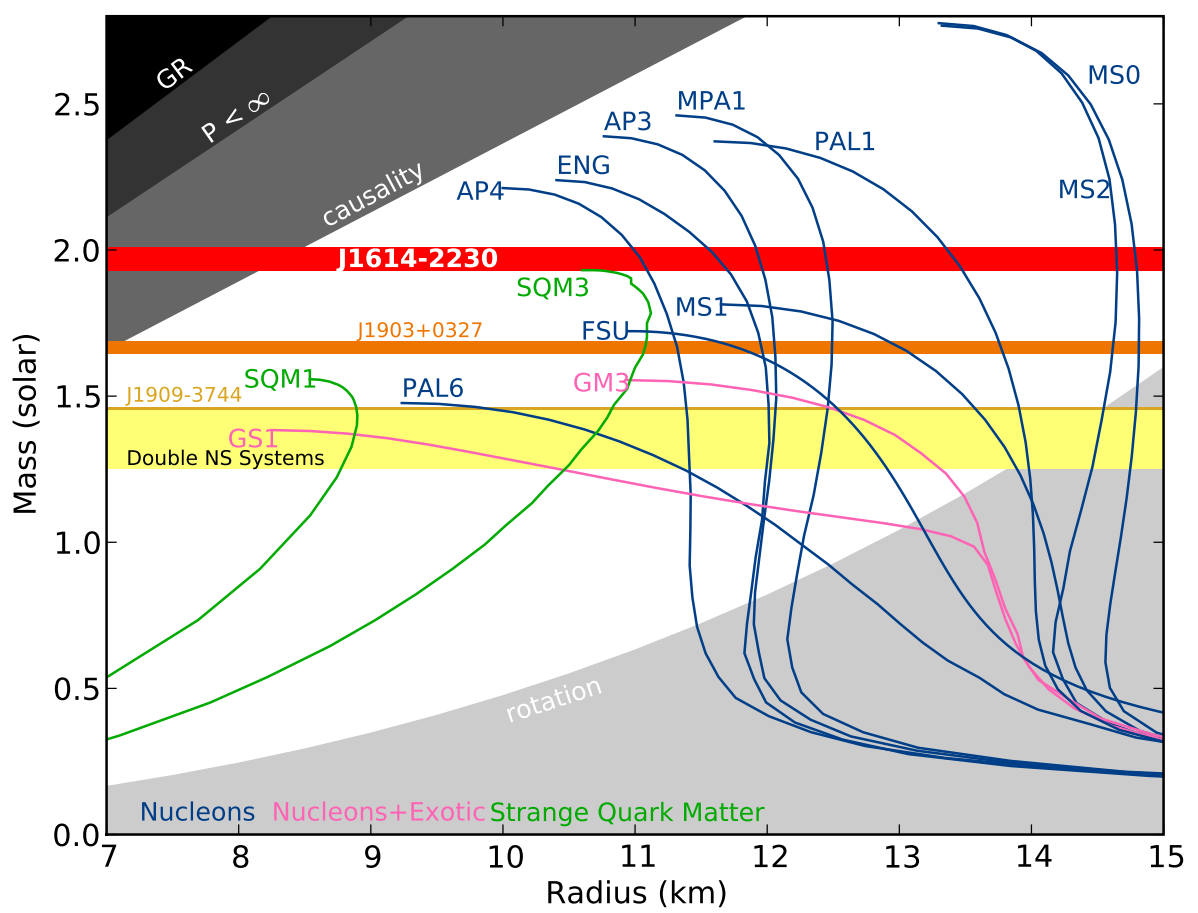

Figura 4.2: Diagrama massa-raio para diferentes EOS [130].

Oppenheimer-Volkoff (TOV), que descrevem a estrutura de uma estrela compacta estática e sem rotação:

$$
\begin{gathered}
\frac{d p}{d r}=-\frac{1}{r^{2}}[\epsilon(r)+p(r)]\left[M(r)+4 \pi r^{3} p(r)\right]\left[1-\frac{2 M(r)}{r}\right]^{-1}, \\
\frac{d M(r)}{d r}=4 \pi r^{2} \epsilon(r) .
\end{gathered}
$$

onde $M(r), p(r)$ e $\varepsilon(r)$ são respectivamente massa, pressão e densidade de energia em função do raio $r$. A derivação da TOV está no Apêndice E.

$\mathrm{Na}$ dedução de tais equações assumimos que o meio estelar é isotrópico. Porém, quando existe um campo magnético externo, ele define uma direção privilegiada quebrando a isotropia. Na prática a quebra da isotropia é um efeito pequeno, exceto 
no limite em que o campo magnético é muito intenso. A região de valores de $\vec{B}$ em que ela se torna importante depende da equação de estado utilizada. A estimativa quantitativa da anisotropia é feita através da comparação entre a pressão paralela ao campo magnético e a pressão perpendicular a ele. Como pode ser visto na Figura 4.3, apenas para campos maiores do que $10^{17} \mathrm{G}$ vemos diferença entre as pressões [136].

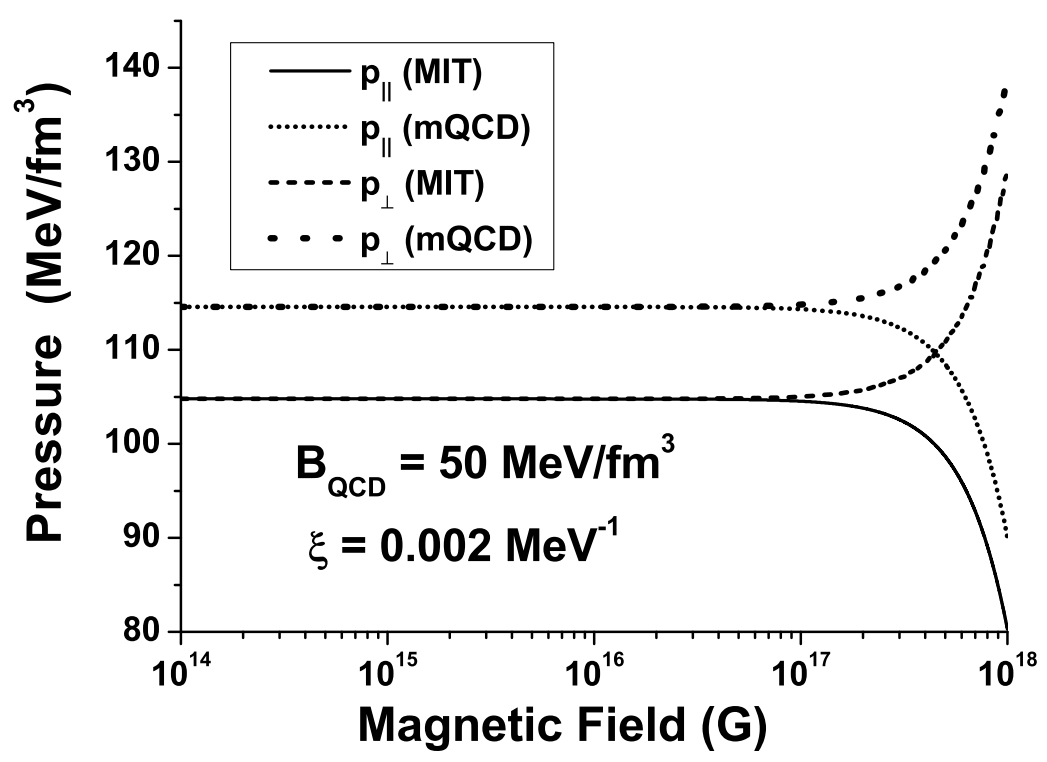

Figura 4.3: Pressões paralela e perpendicular ao campo magnético. As linhas superiores mostram os resultados da $\mathbf{m} Q C D,\left(\xi=g / m_{G}\right)$ e as linhas inferiores os resultados obtidos com a EOS do MIT.

\subsubsection{Janela de Estabilidade e Diagrama Massa-Raio}

Como foi visto, as condições (4.1) à (4.5) devem ser satisfeitas para que o objeto compacto continue no estado de QGP e não hadronize. As massas dos quarks estão fixas nos valores mais aceitos: $m_{u}=5 \mathrm{MeV}, m_{d}=7 \mathrm{MeV}$ e $m_{s}=150 \mathrm{MeV}$. Devemos escolher valores de $\xi=g / m_{g}$ e de $\mathcal{B}_{Q C D}$ tais que as condições mencionadas acima sejam satisfeitas. Na Figura 4.4 mostramos as regiões de valores de $\xi$ e de $\mathcal{B}_{Q C D}$ 
que garantem a estabilidade. Estas regiões, que chamamos de janelas de estabilidade são aquela compreendida entre a linha tracejada e a linha cheia e também aquela compreendida entre a linha pontilhada e a linha traço-ponto.

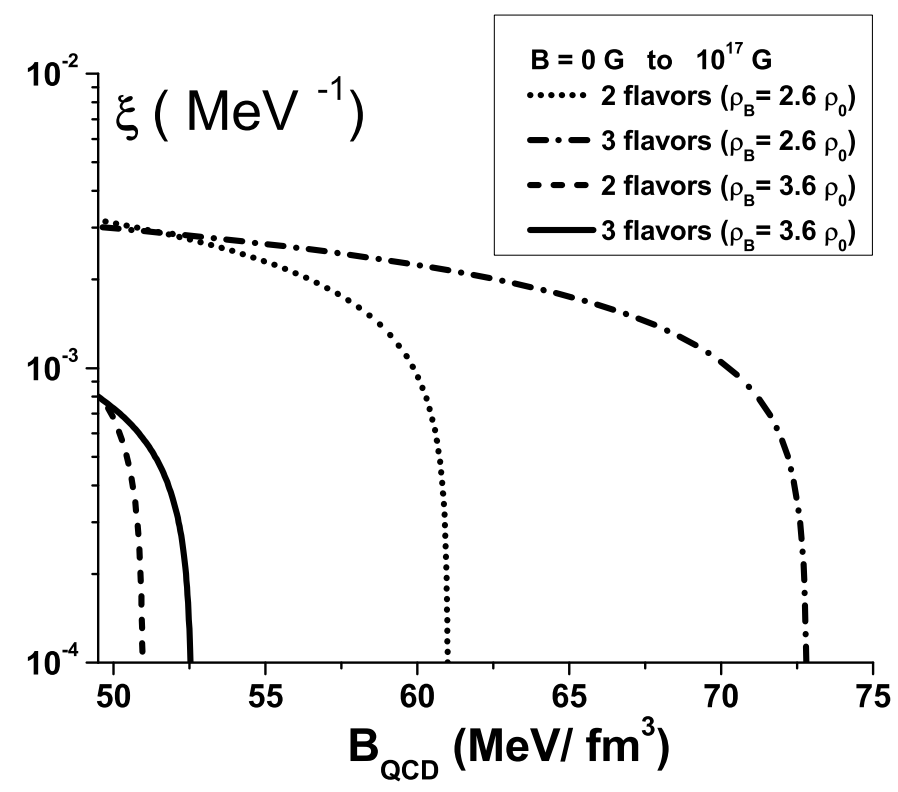

Figura 4.4: Janela de estabilidade definidas pelas condições (4.1) à (4.5).

Existe um valor máximo para a densidade bariônica, $\rho_{B} \simeq 3.7 \rho_{0}$, além do qual não encontramos mais nenhuma janela de estabilidade. Isto é esperado, pois caso $\rho_{B}$ aumente e comece a ser dominante na expressão para a densidade de energia (2.74), a razão $\varepsilon / \rho_{B}$ irá crescer e não conseguirá mais satisfazer o lado esquerdo de (4.5). Na Figura 4.5 vemos claramente os valores mínimo $\left(\rho_{B} / \rho_{0}>1.5\right)$ e máximo $\left(\rho_{B} / \rho_{0}<3.7\right)$ da razão $\rho_{B} / \rho_{0}$ e também o valor máximo do campo magnético de $5 \times 10^{18} G$ para que exista estabilidade. Para a equação de estado do modelo de sacola do MIT, encontramos uma figura muito semelhante.

Com os limites que foram encontrados para os parâmetros $\xi$ e de $\mathcal{B}_{Q C D}$ integramos numericamente as equações de TOV (4.6) e (4.7) e encontramos as funções $p(r)$ e $M(r)$. Usamos as equações de estado do modelo de sacola do MIT e da mQCD em temperatura nula e com campo magnético finito. No centro da estrela $(r=0)$, temos 


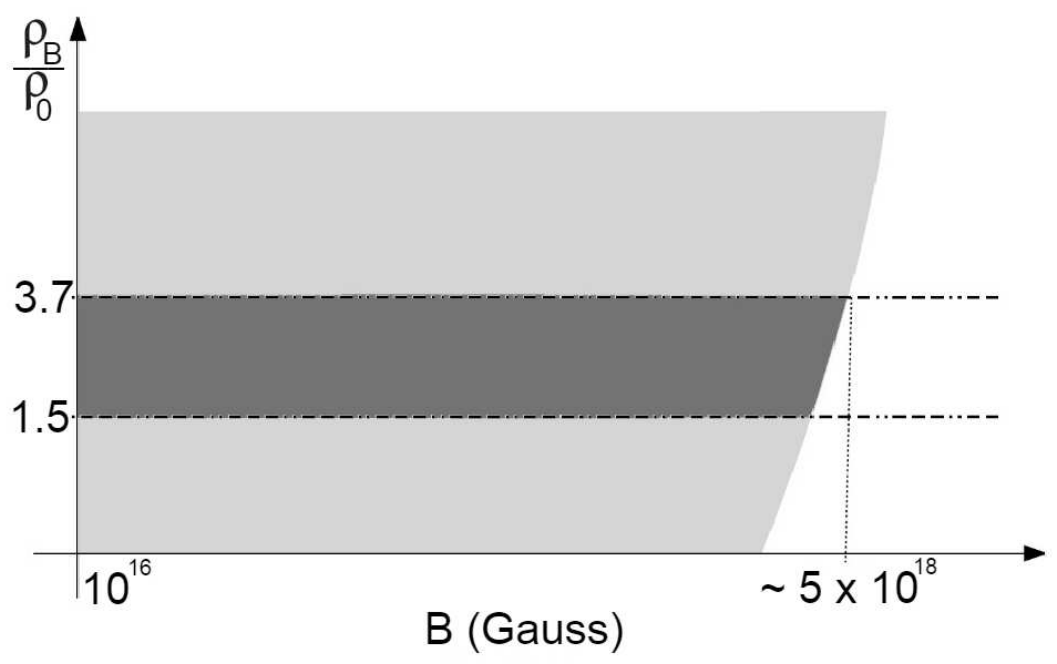

Figura 4.5: Diagrama de estabilidade: densidade bariônica (dividida por $\rho_{0}$ como função do campo magnético. A região cinza claro satisfaz (4.1), (4.3) e (4.4). A região em cinza escuro satisfaz também (4.5).

$M(r=0)=0$ e $p(r=0)=p$, onde $p$ é a pressão dada pela EOS usada. Precisamos também de $\varepsilon(r=0)=\varepsilon$, que é a densidade de energia dada pela equação de estado. A integração termina quando a pressão chega a zero, que indica a superfície da estrela.

A Figura 4.6 mostra a diferença entre os diagramas Massa-Raio obtidos com a mQCD e com a EOS do modelo de sacola do MIT para campo magnético igual a zero e igual a $5 \times 10^{16} G$. A diferença entre as curvas é imperceptível e assim ainda podemos utilizar $p_{\|}=p_{\perp}$. Claramente, vemos que a mQCD fornece valores maiores para a massa da estrela. A maior massa é de $2.05 M_{\odot}$ para a mQCD e de $1.89 M_{\odot}$ para o MIT.

Quando aumentamos o campo magnético, temos $p_{\|}<p_{\perp}$. Nas Figuras 4.7 e 4.8 apresentamos as curvas com as duas pressões. Na primeira destas figuras mantemos $\xi$ fixo e variamos $\mathcal{B}_{Q C D}$. Concluímos que $\mathcal{B}_{Q C D}$ maior leva a uma massa máxima menor. Para $\mathcal{B}_{Q C D}=53 \mathrm{MeV} / \mathrm{fm}^{3}$ temos $2.22 \mathrm{M}_{\odot}$ e $2.04 \mathrm{M}_{\odot}$. Para $\mathcal{B}_{Q C D}=61 \mathrm{MeV} / \mathrm{fm}^{3}$ temos $2.06 M_{\odot}$ e $1.93 M_{\odot}$. Na Figura 4.8 mantemos $\mathcal{B}_{Q C D}$ fixo e variamos $\xi$. Neste 
caso o aumento de $\xi$ fornece massas maiores, para $\xi=0.0015 \mathrm{MeV}^{-1}$ temos $2.20 \mathrm{M}_{\odot}$ e $2.06 M_{\odot}$ e para $\xi=0 \mathrm{MeV}^{-1}(\mathrm{MIT})$ temos $2.08 M_{\odot}$ e $1.92 M_{\odot}$, resultados que estão qualitativamente de acordo com os encontrado em [34] que são para um campo magnético nulo. Aumentando ainda mais o campo magnético, observamos o efeito da anisotropia das pressões, como mostra a Figura 4.9. Quando o campo é de $5 \times 10^{17} G$ a diferença entre a massa máxima e a mínima está em torno de $10 \%$.

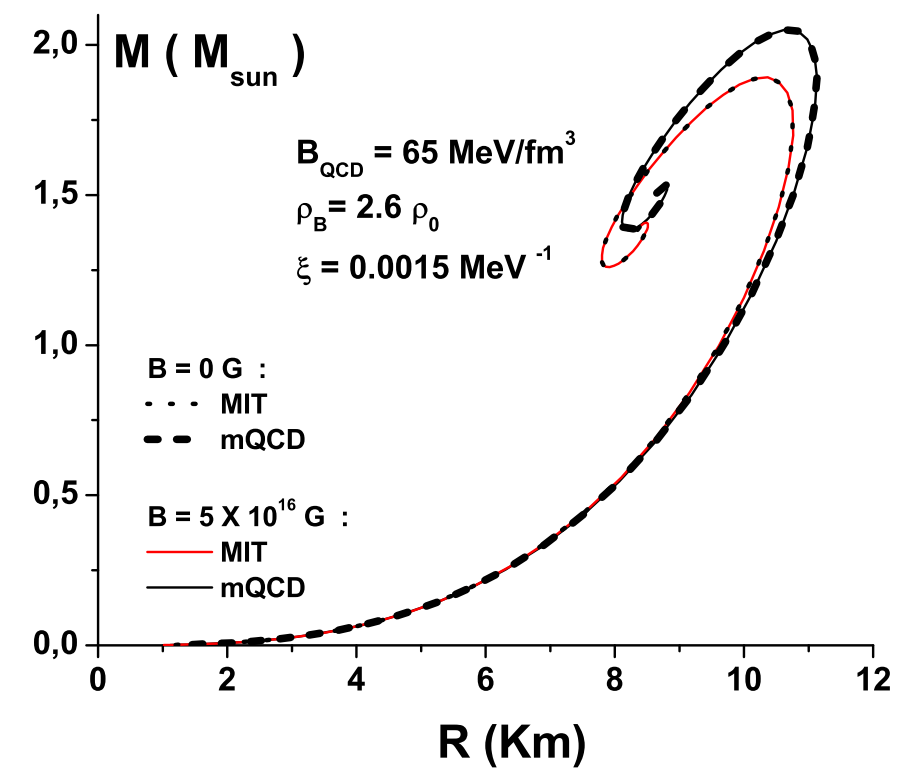

Figura 4.6: Curvas Massa-Raio obtidas com a EOS do MIT e da mQCD, para dois valores do campos magnético. $\mathcal{B}_{Q C D}$ e $\xi$ satisfazem o critério de estabilidade. A densidade central é $\rho_{B}=2.6 \rho_{0}$.

Ao impormos a condição de estabilidade da estrela determinamos um conjunto de valores dos parâmetros que garantem o equilíbrio químico e estabilidade no caso da mQCD e outro conjunto de valores no caso do modelo de sacola do MIT. Quando aumentamos o valor do campo magnético a janela de estabilidade diminui e desaparece completamente quando $B=5 \times 10^{18} G$. Para valores maiores do que este o formalismo da TOV já não se aplica e devemos retomar o formalismo desde o início e procurar soluções das equações de Einstein na presença de um campo magnético externo. 


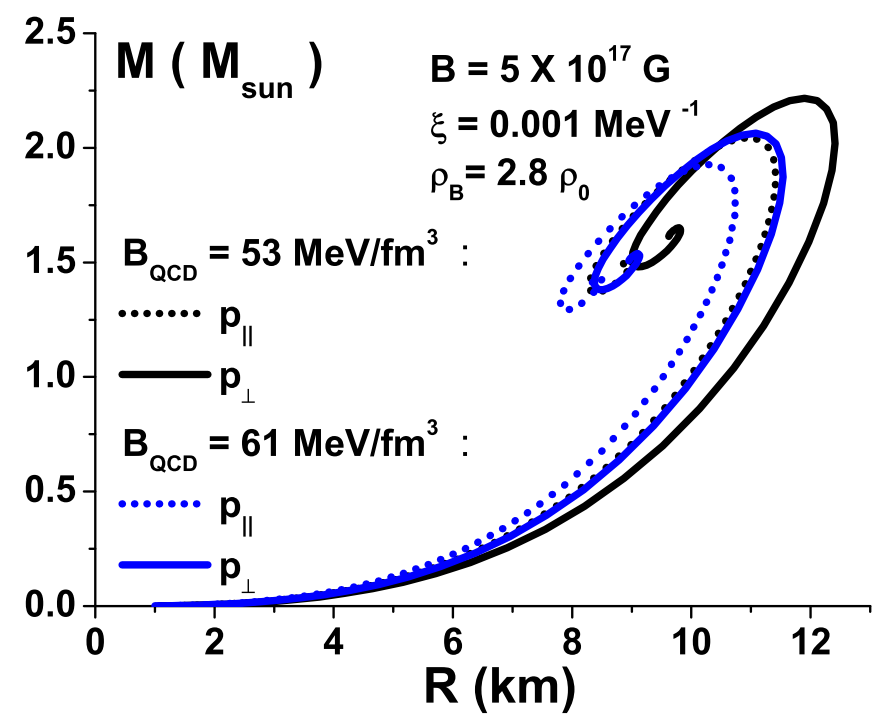

Figura 4.7: Curvas Massa-Raio, obtidas com a mQCD variando $\mathcal{B}_{Q C D}$ e mantendo $\xi$ fixo.

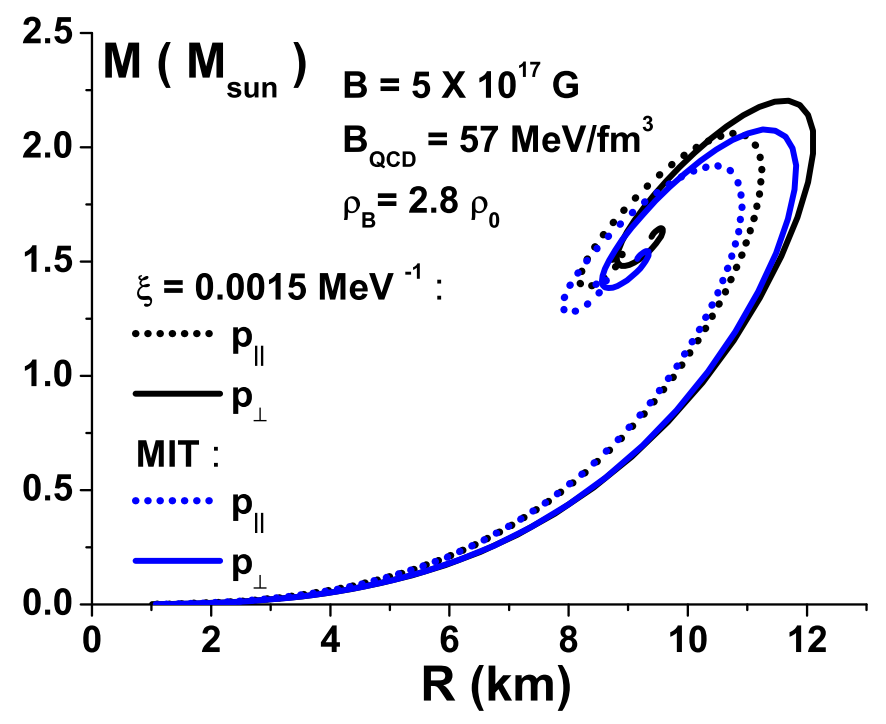

Figura 4.8: Curvas Massa-Raio, obtidas com a $m Q C D$, variando $\xi$ (MIT indica $\xi=0$ ) e mantendo $\mathcal{B}_{Q C D}$ fixo. 


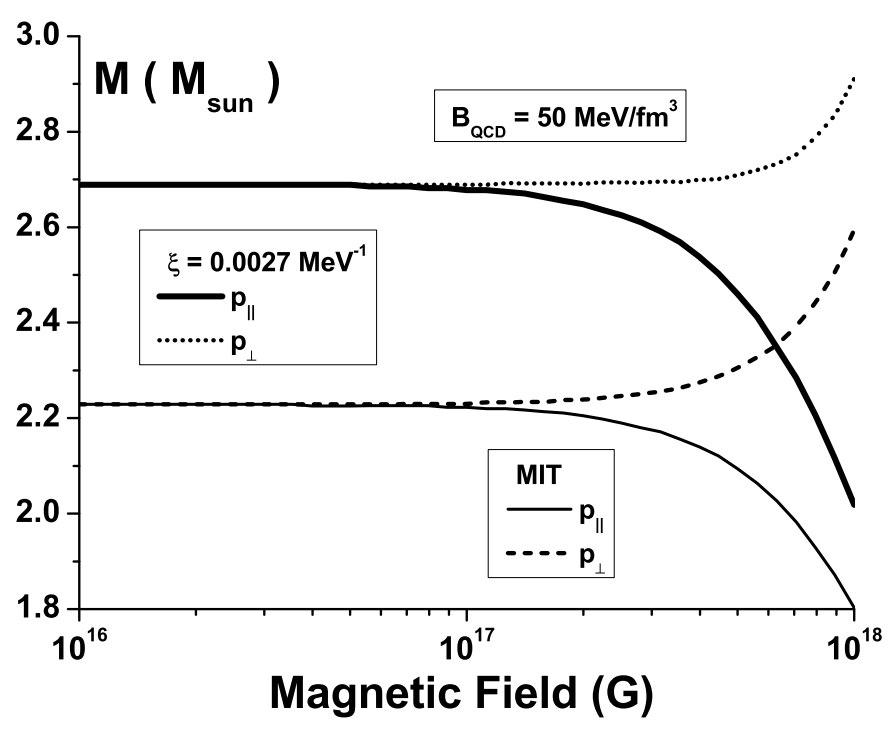

Figura 4.9: Efeito da anisotropia das pressões sobre a massa da estrela calculada pela TOV. Os potenciais químicos satisfazem o critério de estabilidade e são: $\nu_{u}=300 \mathrm{MeV}, \nu_{d}=\nu_{s}=316.5 \mathrm{MeV}$ e $\mu_{e}=16.5 \mathrm{MeV}$.

Ao estudar estrelas de quarks com campos magnéticos, a questão mais interessante é: "Qual é o efeito do campo magnético sobre a massa da estrela?". Nós mostramos que, no formalismo simplificado da TOV, infelizmente não é possível responder esta questão, pois exatamente quando o campo passa a ser importante o formalismo deixa de ser válido. Apesar de suas limitações nosso estudo nos leva a uma interessante conjectura: a de que para campos magnéticos intensos pode ser que não existam estrelas estáveis feitas somente de matéria de quarks.

Os resultados obtidos com a mQCD são compatíveis com os resultados experimentais de $[130,131]$. Com um campo magnético de $B=5 \times 10^{17} G$ o uso da mQCD gerou massas de até $2.22 M_{\odot}$ e $2.20 M_{\odot}$ para diferentes valores estáveis de $\xi$ e $\mathcal{B}_{Q C D}$. Os raios obtidos ficaram em torno de $\sim 12 k m$, e são valores bem compatíveis com outras expectativas teóricas. 


\subsection{Universo Primordial}

O melhor modelo para explicar a origem do Universo é o modelo do Big Bang. De acordo com ele, no início, a matéria se encontrava num estado extremamente quente e denso. Com o passar do tempo ela se expandiu e se resfriou até chegar ao presente, passando por uma fase onde a matéria hadrônica estava desconfinada e formava um plasma quente de quarks e glúons $[31,32,137]$.

Nos modelos cosmológicos baseados no Big Bang a equação de estado deve ser fornecida. Nas últimas duas décadas nosso conhecimento sobre a equação de estado do QGP aumentou muito. Do lado teórico houve grande progresso nos cálculos de QCD na rede (LQCD) e hoje podemos dizer que a EOS do QGP é bem conhecida no regime de densidade bariônica nula e altas temperaturas. Estas são precisamente as condições em que se encontrava o Universo primordial. Ao mesmo tempo este plasma quente foi recriado em laboratório em colisões de íons pesados relativísticos feitas no RHIC e no LHC. A análise destes dados ajuda a impor vínculos sobre a EOS do QGP e reduzir as dúvidas que ainda pairam sobre este tema. Este é portanto um bom momento para refazer com as equações de estado modernas os cálculos já feitos em cosmologia com EOS antigas. Em particular poderíamos responder as perguntas: "Como as colisões de íons pesados relativísticos mudararm e ainda podem mudar o nosso conhecimento sobre cosmologia?" e "Como os cálculos de QCD na rede mudarão e ainda podem mudar o nosso conhecimento sobre cosmologia?". Sobre esta última questão podemos dizer o seguinte: a grande maioria de cálculos cosmológicos se baseavam na ideia de que a transição de fase quark-hádron era uma transição de primeira ordem. Desde 2010 sabemos que esta transição não é realmente uma transição de fase e sim um "cross-over", um processo suave e sem descontinuidades. Este fato tem implicações diretas sobre a evolução temporal de várias grandezas e em especial para o cálculo da emissão de ondas gravitacionais durante a transição.

A partir de 2011 [138] teve início uma nova rodada de cálculos em cosmologia com 
estas novas equações de estado do QGP. Já em 2011, Florkowski utilizou a nova equação de estado calculada em QCD na rede. Nesta seção nós vamos estudar o impacto de uma nova equação de estado sobre a dinâmica do Universo usando a mQCD. A vantagem da mQCD é que com ela podemos fazer cálculos com potencial químico não nulo, que é a região do diagram de fase da QCD onde não podemos usar LQCD.

A seguir introduzimos de forma sucinta as equações de Friedmann, que descrevem a evolução do Universo. Mais detalhes estão contidos no Apêndice F.

\subsubsection{Equações de Friedmann}

As equações de Friedmann são fundamentais em cosmologia. No Apêndice F apresentamos uma breve derivação delas. Para um "Universo chato" e sem constante cosmológica elas são:

$$
\dot{a}^{2}(t)-\frac{8 \pi G \varepsilon}{3} a^{2}(t)=0
$$

e

$$
\frac{\ddot{a}(t)}{a(t)}=-\frac{4 \pi G}{3}(\varepsilon+3 p)
$$

Derivando a primeira com relação ao tempo e substituindo na segunda encontramos:

$$
\dot{\varepsilon}+3 \frac{\dot{a}(t)}{a(t)}(\varepsilon+p)=0
$$

Resolvendo a equação acima para $\dot{a} / a$ e substituindo em (4.8) [139] temos:

$$
-\frac{d \varepsilon}{3 \sqrt{\varepsilon}(\varepsilon+p)}=\sqrt{\frac{8 \pi G}{3}} d t
$$

Esta é a equação mais importante para nós, pois para uma dada equação de estado $p=p(\varepsilon)$ podemos integrá-la e determinar a evolução temporal da densidade de energia 
$\varepsilon(t)$. Isto será feito na próxima subseção.

\subsubsection{Evolução Temporal}

Nesta seção vamos apresentar as soluções numéricas de (4.11), para as equações de estado do Modelo de Sacola do MIT, da mQCD e de LQCD com e sem potencial químico. Por simplicidade vamos considerar somente dois sabores de quarks, up e down, com massas iguais e no regime de altas temperaturas. Vamos usar a seguinte condição inicial:

$$
\varepsilon_{i}\left(t_{i}\right)=10^{7} \mathrm{MeV} / \mathrm{fm}^{3} \quad \text { em } \quad t_{i}=10^{-9} \mathrm{~s}
$$

Com base nas estimativas feitas em [140], vamos estudar a evolução temporal do Universo desde o tempo da transição de fase eletrofraca, $t_{i}=10^{-9} \mathrm{~s}$, até a transição de fase da QCD (fim do QGP), $t_{f}=10^{-4} s$.

Na Figura 4.10 mostramos a pressão como função da densidade de energia e a velocidade do som para as EOS do MIT e da mQCD $\left(m_{g}=10 \mathrm{MeV}\right)$. Podemos observar que a mQCD é uma EOS mais dura do que o MIT, ou seja, possui uma pressão maior, exceto quando o potencial químico é zero $(\mu=0)$, caso em que reproduz o resultado da EOS do MIT.

Vamos utilizar a equação de estado do Modelo de Sacola do MIT como base para comparações com as outras EOS. Resolvemos (4.11) e mostramos na Figura 4.11 a evolução temporal da densidade de energia e da temperatura neste modelo.

O interessante da mQCD é que com ela podemos fazer os cálculos com um potencial químico finito. Na Figura 4.12 apresentamos a razão da evolução temporal entre a densidade de energia obtida com a mQCD e aquela obtida com o MIT. Apresentamos também a evolução temporal da razão das respectivas temperaturas. Vemos uma pequena diferença entre as previsões dos dois modelos, que aumenta com o valor do potencial químico. 


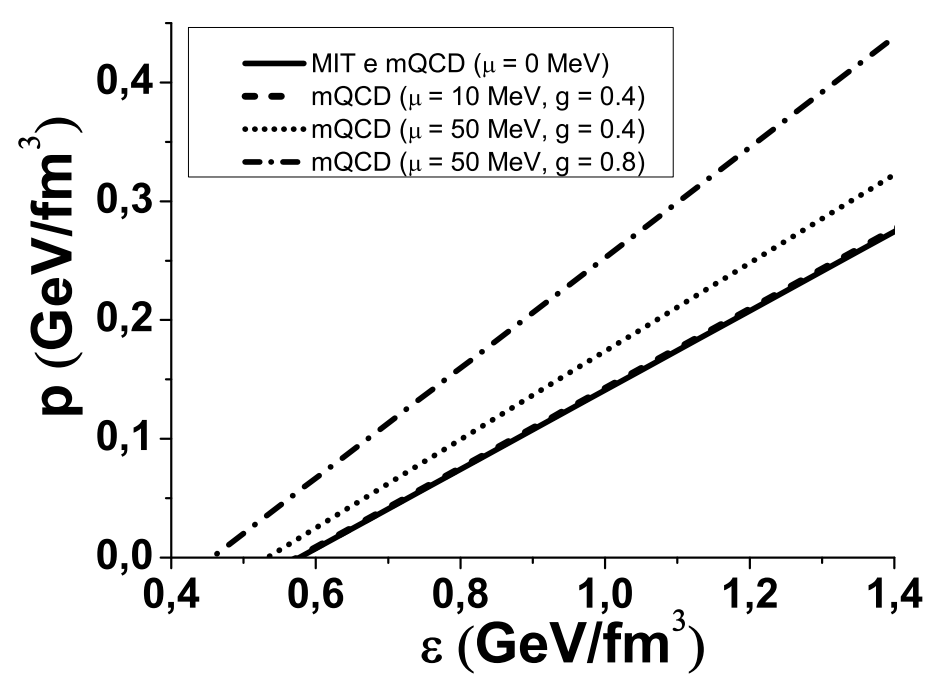

(a)

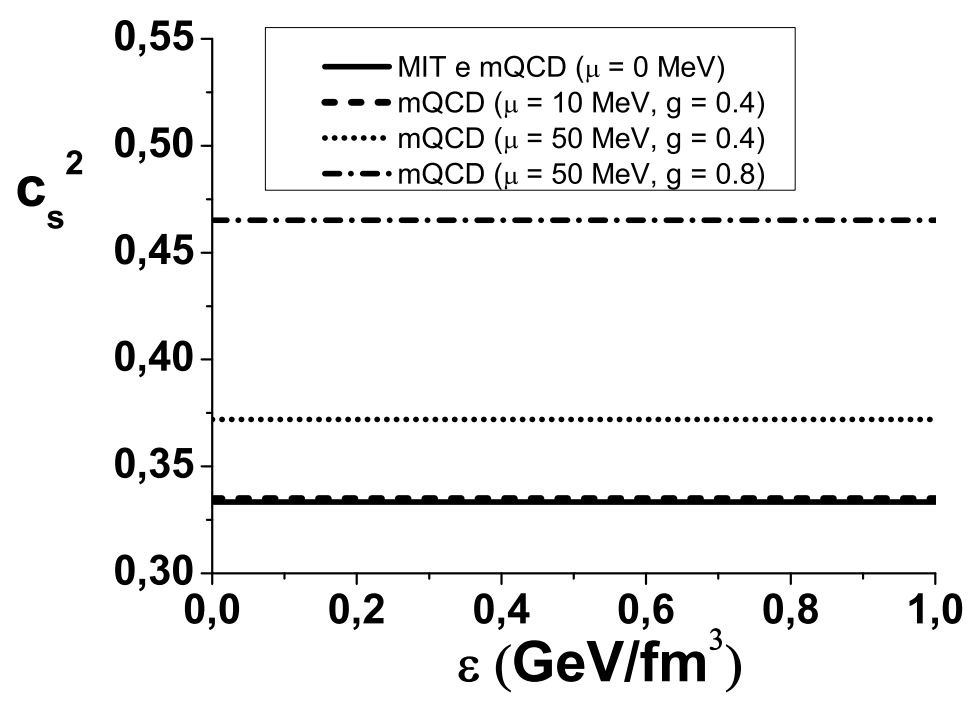

(b)

Figura 4.10: Comparação das EOS do MIT e da mQCD. (a) Equação de estado. (b) Velocidade do som.

As Figuras 4.13(a) e 4.13(b) mostram a evolução temporal da densidade de energia e temperatura para a EOS da LQCD dividida pela densidade de energia obtida com potencial químico nulo. Das curvas concluímos que quando o potencial químico não é muito grande, ele não influencia a evolução temporal. Comparando então o resultado 


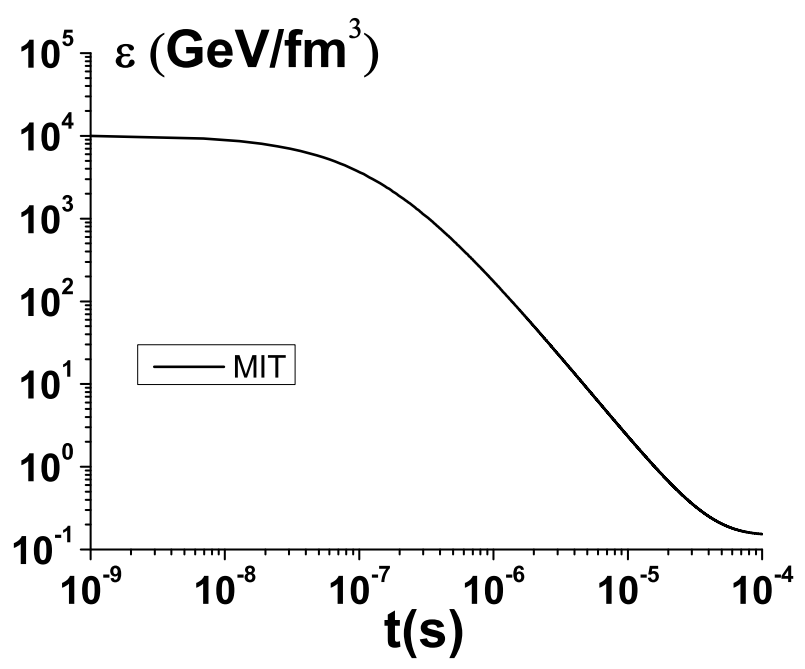

(a)

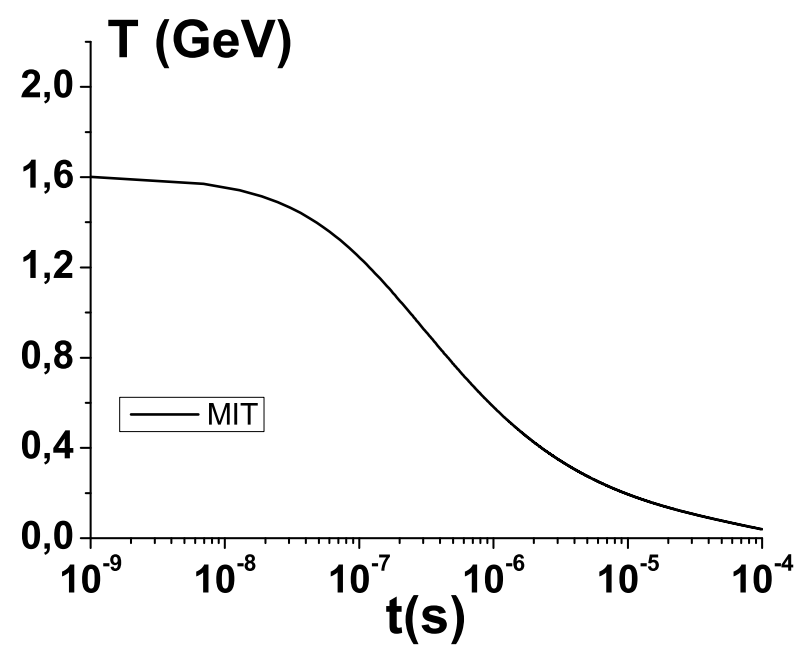

(b)

Figura 4.11: Modelo de sacola do MIT, $\operatorname{com} \mathcal{B}=150 \mathrm{MeV} / \mathrm{fm}^{3}$. (a) Evolução temporal da densidade de energia. (b) Evolução temporal da temperatura.

da LQCD (sem potencial químico) com o do MIT, nas Figuras 4.13(c) e 4.13(d), observamos uma diferença apreciável apenas no final do intervalo temporal considerado. Quando comparamos os resultados da LQCD com os da mQCD com $\mu=50 \mathrm{MeV}$, nas Figuras 4.13(e) e 4.13(f), vemos que os resultados são semelhantes e (como no caso do potencial químico nulo) uma diferença significativa aparece apenas em tempos tardios 


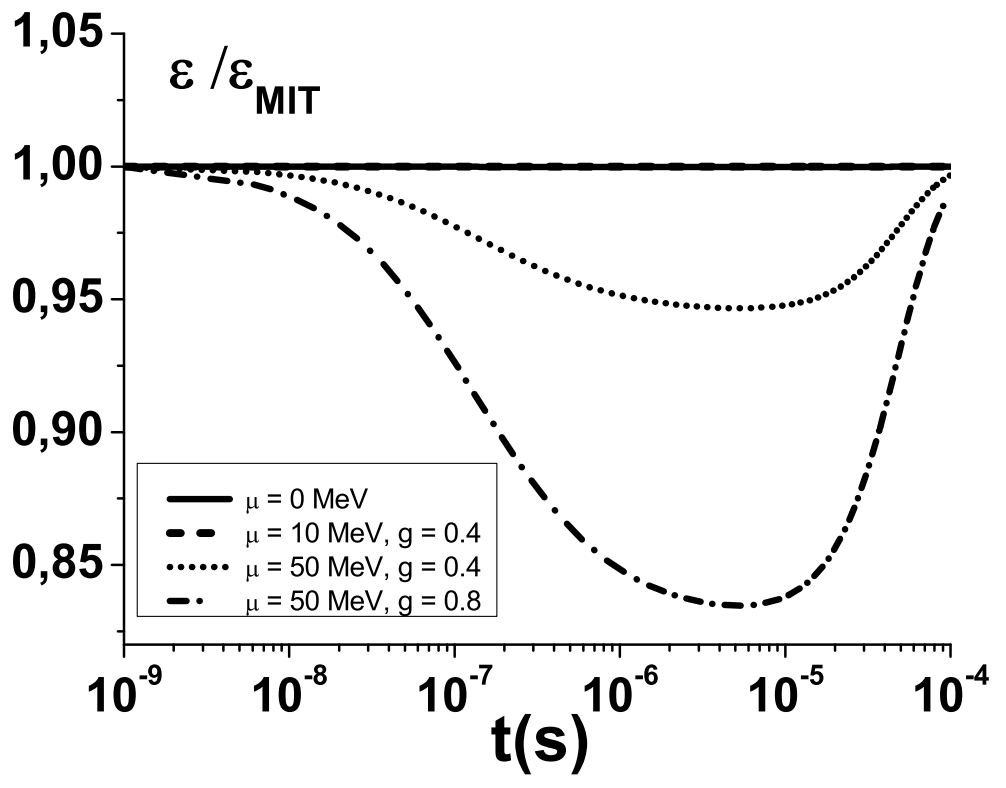

(a)

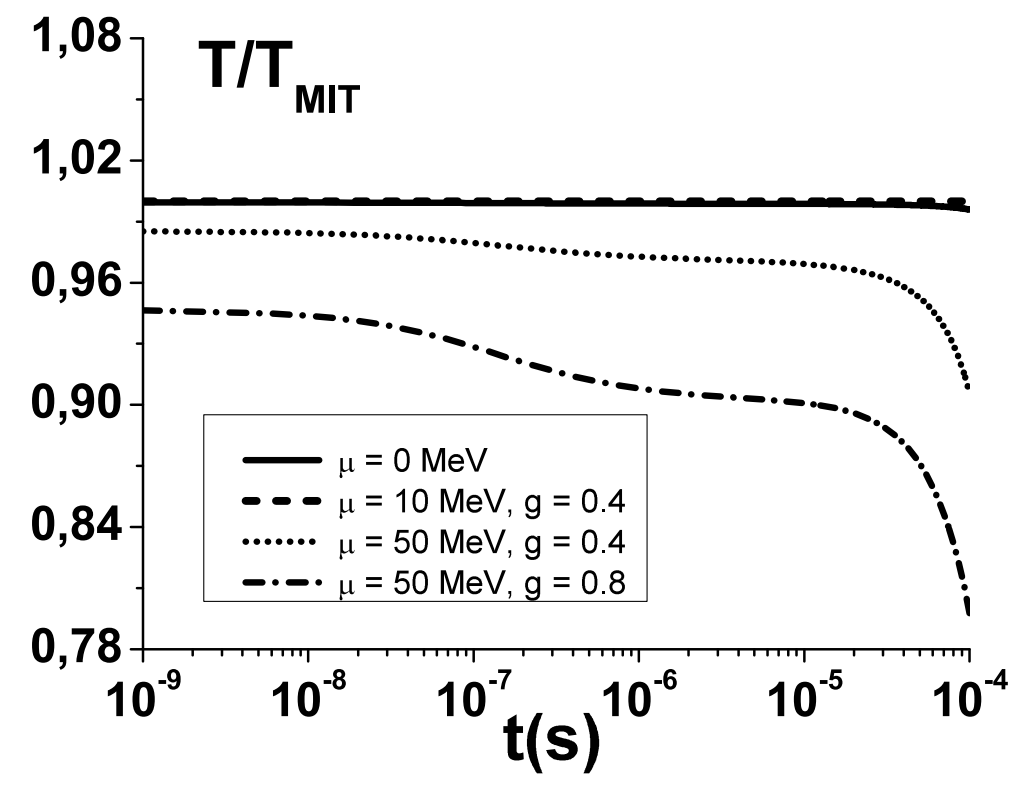

(b)

Figura 4.12: Comparação da mQCD $\left(m_{g}=10 \mathrm{MeV}\right)$ com o MIT. (a) Razão da evolução temporal da densidade de energia. (b) Razão da evolução temporal da temperatura. 
da evolução.

\section{Influência da Matéria Eletrofraca na Evolução Temporal}

Uma das diferenças entre o QGP produzido nas colisões de íons pesados e o que existiu no início do Universo é que este último contém também matéria eletrofraca (EW) que é constituida pelos bósons $Z, W^{+}, W^{-}$e fótons. Para estimar a sua contribuição na evolução temporal vamos utilizar [138,141,142]:

$$
\varepsilon_{e w}=g_{e w} \frac{\pi^{2}}{30} T^{4} \quad \text { e } \quad p_{e w}=g_{e w} \frac{\pi^{2}}{90} T^{4}
$$

onde $g_{e w}=14.45$. A densidade de energia total e a pressão total do sistema, serão dados por:

$$
\varepsilon=\varepsilon_{i}+\varepsilon_{\text {ew }} \quad \text { e } \quad p=p_{i}+p_{\text {ew }}
$$

onde $\varepsilon_{i}$ e $p_{i}$ são respectivamente a densidade de energia e pressão do QGP para o modelo $i$, respectivamente.

Na Figura 4.14 mostramos a contribuição eletrofraca para a EOS da LQCD, onde definimos $R_{\varepsilon}=\varepsilon(t) / \varepsilon_{i}(t)$ e $R_{T}=T(t) / T_{i}(t)$. Das curvas vemos que essa contribuição é significativa somente em tempos tardios, quando o sistema se aproxima da transição quark-hádron.

\section{Evolução Temporal do Fator de Escala}

Podemos reescrever a equação de Friedmann (4.10) como [138, 141-143]:

$$
\frac{\dot{a}(t)}{a(t)}=-\frac{\dot{\varepsilon}(t)}{3[\varepsilon(t)+p(t)]}=\sqrt{\frac{8 \pi G}{3} \varepsilon(t)}
$$

onde $a(t)$ é o fator de escala. Considerando o intervalo de tempo transcorrido desde 


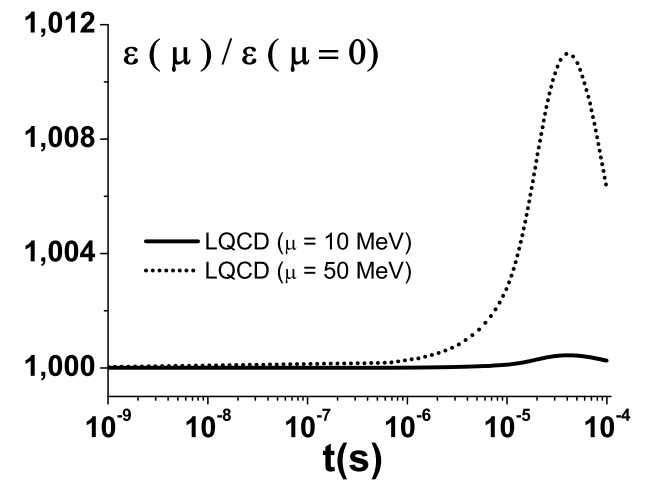

(a)

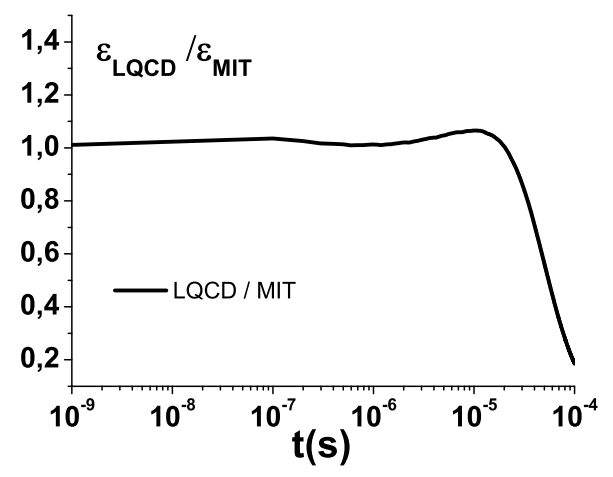

(c)

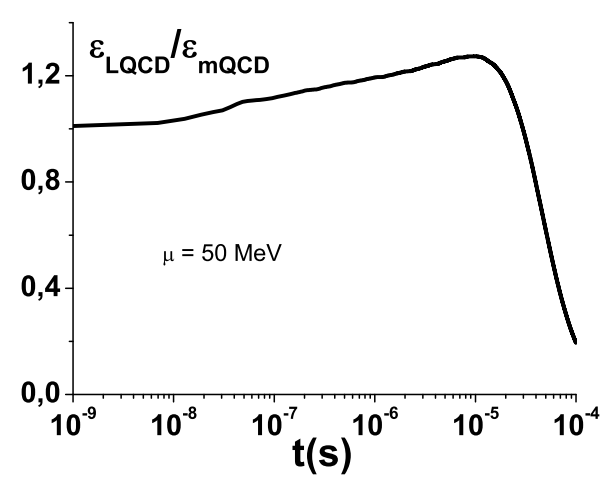

(e)

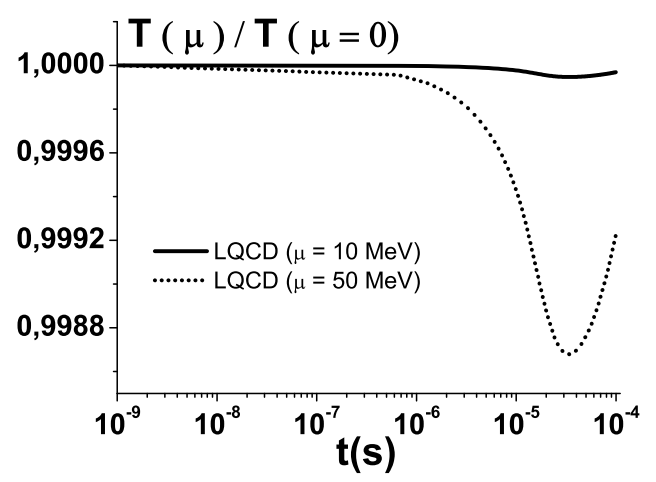

(b)

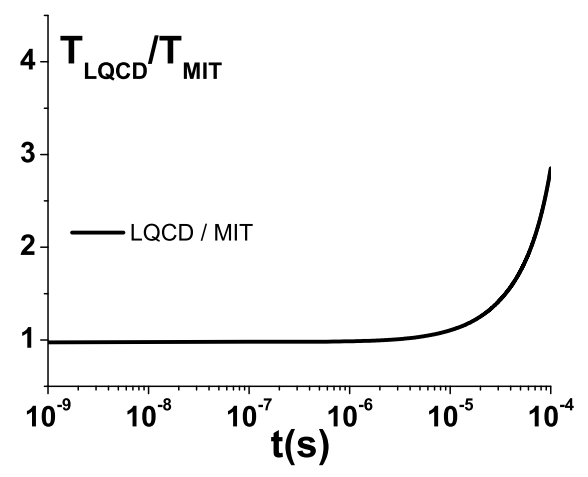

(d)

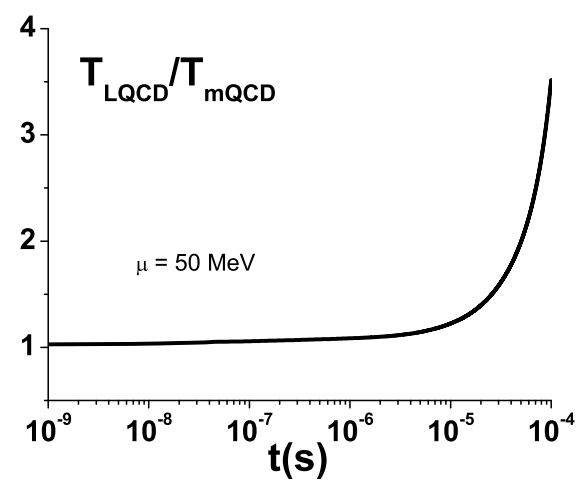

(f)

Figura 4.13: Resultados da EOS da LQCD. (a) Efeito do potencial químico na evolução temporal da densidade de energia. (b) Efeito do potencial químico na evolução temporal da temperatura. (c) Razão LQCD/MIT para densidade de energia. (d) Razão LQCD/MIT para temperatura. (e) Razão LQCD/mQCD para densidade de energia. (f) Razão $L Q C D / m Q C D$ para temperatura. 


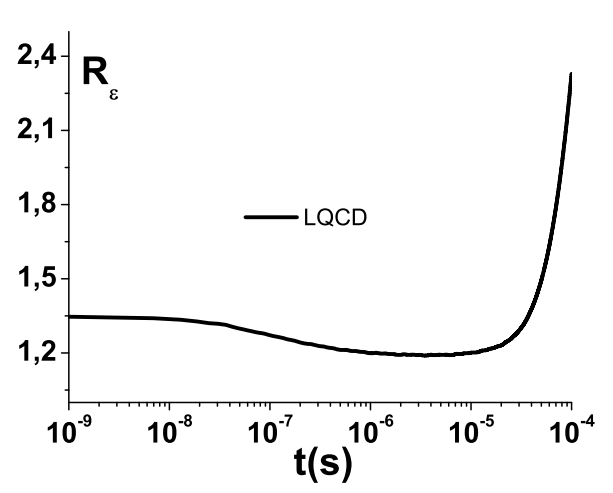

(a)

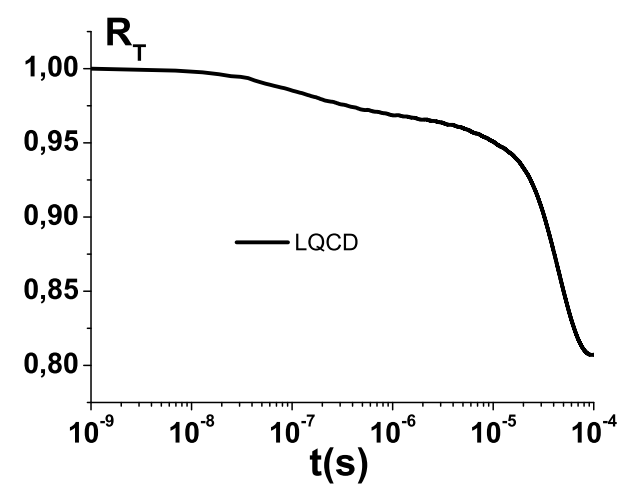

(b)

Figura 4.14: Contribuição EW para a evolução temporal com a EOS da LQCD. (a) Evolução temporal da densidade de energia. (b) Evolução temporal da temperatura.

a formação do Universo, $t=0$, até o "tempo inicial" de nosso interesse $t_{0}$, temos $\varepsilon(t=0) \equiv \tilde{\varepsilon}$ e $\varepsilon\left(t=t_{0}\right) \equiv \varepsilon_{0}$. A densidade de energia em $t=0$ é muito maior que em $t=t_{0}$, ou seja, $\tilde{\varepsilon}>>\varepsilon_{0}$. Seguindo o que foi feito em [138] assumimos que a velocidade do som é constante no tempo e assim $p(t)=c_{s}^{2} \varepsilon(t)$.

Integrando a segunda igualdade em (4.15) e resolvendo para $t_{0}, \operatorname{com}(1 / \sqrt{\tilde{\varepsilon}}) \rightarrow 0$, encontramos:

$$
t_{0}=\frac{1}{\sqrt{6 \pi G\left(1+c_{s}^{2}\right)^{2} \varepsilon_{0}}}
$$

Vamos agora considerar o intervalo de tempo transcorrido entre o tempo inicial $t_{0}$, quando a densidade de energia era $\varepsilon_{0}$, até um tempo genérico $t$ quando a densidade de energia é $\varepsilon$. Integramos novamente a segunda igualdade de (4.15) neste intervalo de tempo, com (4.16). Resolvendo para $\varepsilon(t)$, temos:

$$
\varepsilon(t)=\frac{1}{6 \pi G\left(1+c_{s}^{2}\right)^{2} t^{2}}
$$

Conhecendo a dependência temporal da densidade de energia, voltamos em (4.15) e 
integramos de $t_{0}$ até $t$, obtendo:

$$
\frac{a(t)}{a\left(t_{0}\right)}=\left(\frac{t}{t_{0}}\right)^{\frac{2}{3\left(1+c_{s}^{2}\right)}}
$$

Em [138] foi obtida a expressão para o caso particular em que $c_{s}^{2}=1 / 3$. Neste caso temos $a / a\left(t_{0}\right)=\left(t / t_{0}\right)^{1 / 2}$. Na Figura 4.15 temos a razão $a / a\left(t_{0}\right)$ como função do tempo. Usando a equação de estado do MIT como referência, comparamos a evolução do fator de escala obtida com a EOS da LQCD, com a da mQCD e com a do MIT, com a contribuição EW. Como é claro de (4.18), a única diferença nestas curvas vem da velocidade do som, que é diferente para cada modelo, mas constante no tempo. Um caso limite é quando $c_{s}^{2}=0$. Neste caso a expansão é mais rápida. No outro limite, $c_{s}^{2}=1$, a expansão é mais lenta. Nos casos considerados, maior pressão leva a uma velocidade do som, $c_{s}^{2}$, maior e a uma taxa de expansão menor.

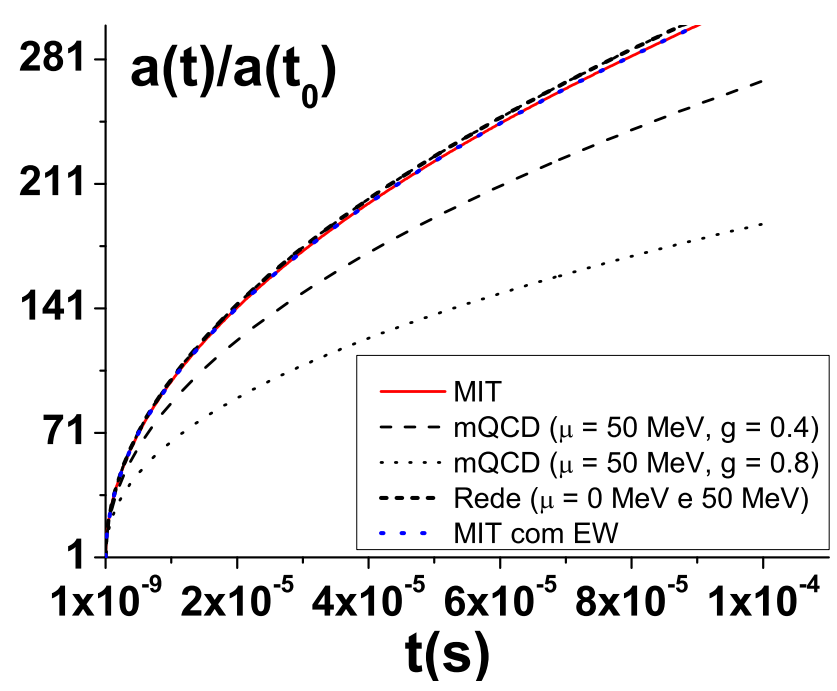

Figura 4.15: Fator de escala como função do tempo para os modelos MIT, LQCD (Rede) e $\mathbf{m Q C D}\left(m_{g}=10 \mathrm{MeV}\right)$.

Nós estudamos a equação de estado do modelo do MIT, que serve como referência de estudo, por causa de sua simplicidade e de sua popularidade. Consideramos também 
outras equações de estado com diferentes ingredientes dinâmicos. Cada ingrediente novo presente numa EOS poderia em princípio gerar alguma modifição drástica na evolução temporal do sistema considerado. Os diferentes modelos estudados foram inseridos nas equações de Friedmann, e estas foram resolvidas numericamente. A principal conclusão é que não houve nenhuma mudança significativa das grandezas estudadas no intervalo de tempo considerado. Concluímos assim que a densidade de energia é pouco sensível aos fatores considerados: o aumento do potencial químico, a mudança dos graus de liberdade do QGP (inclusão de mais sabores de quarks, como o quark s), a intensidade dos condensados de glúons, que geram a massa dinâmica do glúon e a intensidade do campo médio de glúons "hard", que aumenta a dureza da equação de estado considerada. No caso da temperatura, mudanças nestes fatores alteram ainda menos a evolução temporal. Em particular, a inclusão de mais sabores de quarks não produz nenhum efeito visível. 


\subsection{Evolução de Bolhas de QGP em um Gás de Hádrons}

Como foi ilustrado na Figura 1.1 da Introdução, o QGP pode ser produzido a baixas temperaturas e grandes densidades, condições que serão atingidas em futuros experimentos do FAIR [144] e NICA [145]. Depois de sua formação o plasma se expande, se resfria e então hadroniza, criando os hádrons observáveis. Um dos aspectos dessa transição é a passagem do QGP para a matéria hadrônica, um gás de hádrons (HG), em baixas temperaturas e altas densidades bariônicas.

Não existem cálculos com LQCD, mas cálculos com vários modelos indicam que a baixas temperaturas e altos potenciais químicos (altas densidades bariônicas) a transição quark-hádron é uma transição de fase de primeira ordem e a teoria de nucleação pode ser aplicada. A nucleação (ou formação de bolhas) de uma fase de quarks em um meio de matéria hadrônica foi estudada extensivamente na física de íons pesados relativísticos, onde podemos ter a formação de bolhas de QGP em um gás de hádrons [146-150] e também, ao final destas colisões, a formação de bolhas de um gás de hádrons em um meio de QGP [151,152]. Há vários estudos dedicados ao cálculo da tensão superficial, energia livre, raio crítico e taxa de nucleação [153]. No contexto de colisões de íons pesados existe uma extensa literatura sobre o processo de nucleação $[154,155]$. Porém há poucos trabalhos sobre a evolução espaço-temporal destas bolhas $[156,157]$. Esta evolução é descrita por equações diferenciais baseadas em variantes da equação de Rayleigh [158] e da equação de Rayleigh-Plesset (RP) [159].

Uma ilustração qualitativa deste fenômeno é apresentada na Figura 4.16, onde mostramos uma colisão núcleo-núcleo a energias intermediárias (de alguns GeV por par de nucleons no sistema do centro de massa). Dois núcleos colidem (Figura 4.16(a)). Depois da colisão forma-se um sistema composto de matéria hadrônica (H). O sistema possui alta densidade e pressão e assim bolhas de QGP podem se formar e crescer (Figura 4.16(b)). A seguir ocorre a transição de fase e o plasma se torna dominante 
exceto em regiões onde a fase hadrônica persiste. Estas regiões são representadas por bolhas de hádrons que começam a se contrair, Figura 4.16(c). No final da colisão, após a expansão e resfriamento do plasma, ocorre o processo inverso ao mostrado na Figura. Bolhas de gás hadrônico se formam, crescem e transformam as regiões de plasma em bolhas, que se contraem. Quando estas últimas desaparecem, a transição para a fase hadrônica está completa.

a)

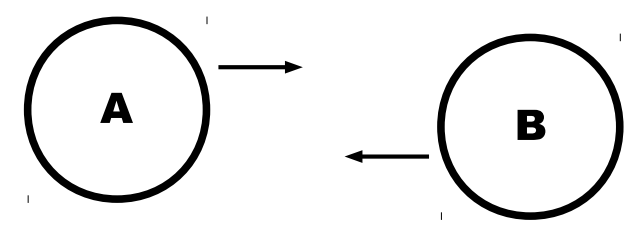

b)

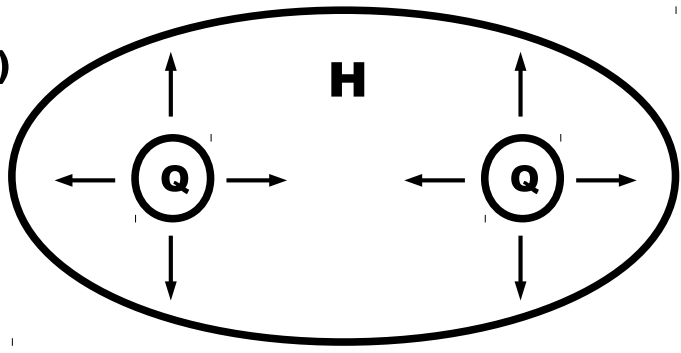

c)

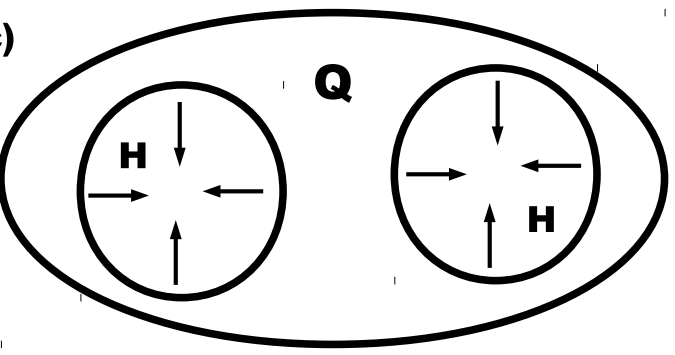

Figura 4.16: Esquema do estágio inicial de uma colisão em energias intermediárias. (a) Dois núcleos colidem. (b) Bolhas de QGP (Q) começam a se formar e crescer em um meio de hádrons $(H)$. (c) $O$ restante de matéria hadrônica está dentro de bolhas que se contraem. No final o QGP é formado. 


\subsubsection{Equação de Rayleigh-Plesset para um Fluido Relativístico}

A equação de Rayleigh-Plesset descreve a dinâmica de uma bolha esférica preenchida com um meio interno (in) imersa em outro meio externo (ex). Sua derivação detalhada se encontra no Apêndice G.1, onde utilizamos as mesmas aproximações feitas em [160]. A equação relativística de Rayleigh-Plesset (RRP) em temperatura finita é dada por:

$$
\frac{d}{d t}\left[\left(I_{1}+I_{2}\right) R^{3} \dot{R}\right]+\left(I_{1}-2 I_{2}\right) R^{2} \dot{R}^{2}=F
$$

onde as integrais são $(\operatorname{com} x=r / R)$ :

$$
\begin{aligned}
I_{1} & \equiv \int_{0}^{1} d x x^{4}\left(\varepsilon_{i n}+p_{i n}{ }^{e f}\right) \gamma_{i n}{ }^{2} \\
I_{2} & \equiv \int_{1}^{\infty} \frac{d x}{x^{2}}\left(\varepsilon_{e x}+p_{e x}{ }^{e f}\right) \gamma_{e x}{ }^{2}
\end{aligned}
$$

sendo $\varepsilon$ e $p$ respectivamente a densidade de energia e pressão. A "pressão efetiva" é:

$$
p_{e x}^{e f} \equiv p_{e x}-\left.T_{e x} \frac{\partial p_{L}}{\partial T}\right|_{T=T_{e x}} \quad \text { e } \quad p_{i n}{ }^{e f} \equiv p_{i n}-\left.T_{i n} \frac{\partial p_{G}}{\partial T}\right|_{T=T_{i n}}
$$

com $T_{e x}$ a temperatura do meio externo e $T_{i n}$ a temperatura no interior da bolha.

Na derivação da RRP, assumimos que o perfil da velocidade é do tipo Hubble (como em [160]):

$$
v_{\text {in }}(r)=\frac{r}{R} \dot{R} \quad \text { para } \quad 0<r<R
$$

$\mathrm{e}$

$$
v_{e x}(r)=\frac{R^{2}\left(R_{\infty}^{3}-r^{3}\right)}{r^{2}\left(R_{\infty}^{3}-R^{3}\right)} \dot{R} \quad \text { para } \quad R<r<R_{\infty}
$$

onde $\dot{R}$ denota a derivada temporal do raio da bolha, $R(t)$, e $R_{\infty}$ é o raio do fluido onde a bolha se forma (que é considerado muito grande). Estas variáveis são usadas 
nos fatores de Lorentz:

$$
\gamma_{i n}=\frac{1}{\sqrt{1-\left(\frac{r}{R} \dot{R}\right)^{2}}} \quad \text { e } \quad \gamma_{e x}=\frac{1}{\sqrt{1-\left(\frac{R^{2}\left(R_{\infty}^{3}-r^{3}\right)}{r^{2}\left(R_{\infty}^{3}-R^{3}\right)} \dot{R}\right)^{2}}}
$$

A função $F$ é:

$F \equiv-R^{2}\left[\left.\left(\varepsilon_{i n}-\varepsilon_{e x}\right)\right|_{R}+\left.\left(\varepsilon_{e x}+p_{e x}-T \frac{\partial p_{e x}}{\partial T}\right)\right|_{\infty}\right]+\frac{3}{R} \int_{0}^{R} d r r^{2}\left(\varepsilon_{i n}+p_{i n}-T \frac{\partial p_{i n}}{\partial T}\right)$

Vamos estudar o caso em que a superfície da bolha se move lentamente em comparação com a velocidade do som no meio $\left(\dot{R}<<c_{S} \simeq 0.4-0.6\right)$ [149]. O sistema se ajusta adiabaticamente com as mudanças do raio da bolha e cada fase mantêm sua pressão, que pode ser considerada constante. Nesta aproximação $\varepsilon$ e $p$ são independentes da coordenada radial e a densidade de energia para cada fase possui a forma:

$$
\varepsilon_{e x}(r=R)=\varepsilon_{e x}(r \rightarrow \infty)=\varepsilon_{e x} \quad \text { e } \quad \varepsilon_{i n}(r=R)=\varepsilon_{i n}(r \rightarrow \infty)=\varepsilon_{i n}
$$

Inserindo (4.27) em (4.26) obtemos:

$$
F=\left[\left(p_{i n}-p_{e x}\right)-\left(\left.T_{i n} \frac{\partial p_{i n}}{\partial T}\right|_{T=T_{i n}}-\left.T_{e x} \frac{\partial p_{e x}}{\partial T}\right|_{T=T_{e x}}\right)\right] R^{2}=-\left(p_{e x}^{e f}-p_{i n}^{e f}\right) R^{2}
$$

No caso de temperatura nula, temos $F=-\left(p_{e x}-p_{i n}\right) R^{2}$, como encontrado em [160]. Podemos ver de (4.19) que o lado esquerdo contém uma "aceleração" e o lado direito contêm uma "força". Quando a pressão fora da bolha é maior que a interna, $p_{e x}^{e f}>p_{i n}{ }^{e f}$, a função $F$ terá valores negativos, implicando que a força aponta para o centro da bolha, o que leva ao colapso. Quando $p_{e x}{ }^{e f}<p_{i n}{ }^{e f}$ a bolha se expande.

Como as funções termodinâmicas $\varepsilon$ e $p$ são independentes do tempo e da coordenada 
radial, as integrais em (4.20) e (4.21) podem ser simplificadas:

$$
I_{1}=\left(\varepsilon_{i n}+p_{i n}{ }^{e f}\right) \int_{0}^{1} d x x^{4}{\gamma_{i n}}^{2}=\left(\varepsilon_{i n}+p_{i n}{ }^{e f}\right) \int_{0}^{1} d x \frac{x^{4}}{\left(1-x^{2} \dot{R}^{2}\right)}
$$

e

$$
I_{2}=\left(\varepsilon_{e x}+p_{e x}^{e f}\right) \int_{1}^{\infty} \frac{d x}{x^{2}} \gamma_{e x}^{2}=\left(\varepsilon_{e x}+p_{e x}^{e f}\right) \int_{1}^{\infty} d x \frac{x^{2}}{\left(x^{4}-\dot{R}^{2}\right)}
$$

Estas integrais podem ser reescritas em termos de funções hipergeométricas. Inserindo (4.29) e (4.30) em (4.19), chegamos em:

$$
A R \dot{R}^{2} \ddot{R}+B R \ddot{R}+C \dot{R}^{2}=-\left(p_{e x}^{e f}-p_{i n}{ }^{e f}\right)
$$

onde

$$
\begin{aligned}
& A=\left[\frac{2}{7}\left(\varepsilon_{\text {in }}+p_{\text {in }}{ }^{e f}\right){ }_{2} F_{1}\left(2, \frac{7}{2} ; \frac{9}{2} ; \dot{R}^{2}\right)+\frac{2}{5}\left(\varepsilon_{\text {ex }}+p_{\text {ex }}^{e f}\right){ }_{2} F_{1}\left(2, \frac{5}{4} ; \frac{9}{4} ; \dot{R}^{2}\right)\right] \\
& B=\left[\frac{1}{5}\left(\varepsilon_{\text {in }}+p_{\text {in }}{ }^{e f}\right){ }_{2} F_{1}\left(1, \frac{5}{2} ; \frac{7}{2} ; \dot{R}^{2}\right)+\left(\varepsilon_{\text {ex }}+p_{\text {ex }}{ }^{e f}\right){ }_{2} F_{1}\left(1, \frac{1}{4} ; \frac{5}{4} ; \dot{R}^{2}\right)\right] \\
& C=\left[\frac{4}{5}\left(\varepsilon_{\text {in }}+p_{\text {in }}{ }^{e f}\right){ }_{2} F_{1}\left(1, \frac{5}{2} ; \frac{7}{2} ; \dot{R}^{2}\right)+\left(\varepsilon_{\text {ex }}+p_{\text {ex }}^{e f}\right){ }_{2} F_{1}\left(1, \frac{1}{4} ; \frac{5}{4} ; \dot{R}^{2}\right)\right]
\end{aligned}
$$

\subsubsection{Limite Não Relativístico}

O limite não relativístico da equação RRP (4.19) é obtido expandindo os fatores de Lorentz em torno de pequenos valores de $\dot{R}^{2}$. Com esta expansão e $x=r / R$, encontramos:

$$
\begin{gathered}
I_{1} \cong \frac{1}{R^{5}} \int_{0}^{R} d x x^{4}\left(\varepsilon_{i n}+p_{i n}{ }^{e f}\right)+\frac{\dot{R}^{2}}{R^{7}} \int_{0}^{R} d x x^{6}\left(\varepsilon_{i n}+p_{i n}{ }^{e f}\right) \\
I_{2} \cong R \int_{R}^{\infty} \frac{d x}{x^{2}}\left(\varepsilon_{e x}+p_{e x}{ }^{e f}\right)+R^{5} \dot{R}^{2} \int_{R}^{\infty} \frac{d x}{x^{6}}\left(\varepsilon_{e x}+p_{e x}{ }^{e f}\right)
\end{gathered}
$$


A pressão efetiva e a densidade de energia das fases interna e externa são consideradas como constantes com relação à coordenada radial e assim (4.35) e (4.36) podem ser simplificadas. Substituindo (4.28), (4.35) e (4.36) em (4.19) e mantendo somente os termos lineares em $\dot{R}^{2}$ encontramos:

$$
\left[\frac{4}{5}\left(\varepsilon_{\text {in }}+p_{\text {in }}^{e f}\right)+\left(\varepsilon_{e x}+p_{\text {ex }}^{e f}\right)\right] \dot{R}^{2}+\left[\frac{1}{5}\left(\varepsilon_{\text {in }}+p_{\text {in }}^{e f}\right)+\left(\varepsilon_{\text {ex }}+p_{\text {ex }}^{e f}\right)\right] R \ddot{R}=-\left(p_{\text {ex }}^{e f}-p_{\text {in }}^{e f}\right)
$$

que é o limite não relativístico de (4.19). Ela pode ser reescrita em uma forma mais compacta como:

$$
R \ddot{R}+\alpha \dot{R}^{2}=\beta
$$

onde

$$
\alpha \equiv \frac{\frac{4}{5}\left(\varepsilon_{i n}+p_{i n}^{e f}\right)+\left(\varepsilon_{e x}+p_{e x}^{e f}\right)}{\frac{1}{5}\left(\varepsilon_{i n}+p_{i n}^{e f}\right)+\left(\varepsilon_{e x}+p_{e x}^{e f}\right)} \text { e } \beta \equiv \frac{-\left(p_{e x}^{e f}-p_{i n}^{e f}\right)}{\frac{1}{5}\left(\varepsilon_{i n}+p_{i n}^{e f}\right)+\left(\varepsilon_{e x}+p_{e x}^{e f}\right)}
$$

Usando a transformação de Sundman, método descrito em [162] e detalhado no Apêndice G.2, encontramos a solução analítica de (4.38):

$$
\begin{gathered}
t(R)=\left(\frac{-2 \mathcal{C} \alpha}{\beta}\right)^{1+\frac{1}{2 \alpha}}\left\{\sqrt{\frac{1}{2 \mathcal{C} \alpha^{2} R^{2 \alpha}}+\frac{\beta}{4 \mathcal{C}^{2} \alpha^{3}}}{ }_{2} F_{1}\left(1+\frac{1}{2 \alpha}, \frac{1}{2} ; \frac{3}{2} ; \frac{4 \mathcal{C}^{2} \alpha^{3}}{\beta}\left[\frac{1}{2 \mathcal{C} \alpha^{2} R^{2 \alpha}}+\frac{\beta}{4 \mathcal{C}^{2} \alpha^{3}}\right]\right)\right. \\
\left.+\mathcal{A}_{2} F_{1}\left(1+\frac{1}{2 \alpha}, \frac{1}{2} ; \frac{3}{2} ; \frac{4 \mathcal{C}^{2} \alpha^{3} \mathcal{A}^{2}}{\beta}\right)\right\}
\end{gathered}
$$

onde as constantes são:

$$
\mathcal{C}=\left(v_{0}{ }^{2}-\frac{\beta}{\alpha}\right) \frac{R_{0}{ }^{2 \alpha}}{2} \quad \text { e } \quad \mathcal{A}=\frac{R_{0}{ }^{1 / 4 \alpha}}{2 \mathcal{C} \alpha^{2}} \sqrt{2 \mathcal{C} \alpha^{2}+\alpha \beta R_{0}{ }^{-1 / 2 \alpha}}
$$

O raio inicial da bolha é $R_{0}=R(0)$ e $v_{0}=\dot{R}(0)$ é a velocidade inicial da fronteira da 
bolha. As funções hipergeométricas em (4.40) são dadas por [163]:

$$
{ }_{2} F_{1}(a, b ; c ; z)=\frac{\Gamma(c)}{\Gamma(b) \Gamma(c-b)} \int_{0}^{1} d \xi \frac{\xi^{b-1}(1-\xi)^{c-b-1}}{(1-\xi z)^{a}}
$$

para $\Re(c)>\Re(b)>0$. Quando o raio $R$ tende a zero, temos o tempo de colapso $\left(t_{\text {colapso }}\right)$ da bolha. Definindo as variáveis da primeira função hipergeométrica de (4.40):

$$
w \equiv \frac{4 \mathcal{C}^{2} \alpha^{3}}{\beta}\left[\frac{1}{2 \mathcal{C} \alpha^{2} R^{2 \alpha}}+\frac{\beta}{4 \mathcal{C}^{2} \alpha^{3}}\right]
$$

temos $w \rightarrow \infty$ quando $R \rightarrow 0$ e neste regime a função hipergeométrica é:

$$
{ }_{2} F_{1}\left(1+\frac{1}{2 \alpha}, \frac{1}{2} ; \frac{3}{2} ; w \rightarrow \infty\right) \cong-i \frac{\sqrt{\pi}}{2} \frac{\Gamma\left(\frac{1}{2}+\frac{1}{2 \alpha}\right)}{\Gamma\left(1+\frac{1}{2 \alpha}\right)} \frac{1}{\sqrt{w}}
$$

Inserindo (4.44) em (4.40) encontramos:

$t_{\text {colapso }}=\left(\frac{-2 \mathcal{C} \alpha}{\beta}\right)^{1+\frac{1}{2 \alpha}}\left\{\frac{\sqrt{\pi}}{2} \sqrt{\frac{-\beta}{4 \mathcal{C}^{2} \alpha^{3}}} \frac{\Gamma\left(\frac{1}{2}+\frac{1}{2 \alpha}\right)}{\Gamma\left(1+\frac{1}{2 \alpha}\right)}+\mathcal{A}_{2} F_{1}\left(1+\frac{1}{2 \alpha}, \frac{1}{2} ; \frac{3}{2} ; \frac{4 \mathcal{C}^{2} \alpha^{3} \mathcal{A}^{2}}{\beta}\right)\right\}$

Notamos que em (4.40) e em (4.45) $\beta$ deve ser negativo para que a bolha colapse. Isso significa que (de (4.39)) é necessário que $p_{e x}$ ef $^{\text {ef }}>p_{\text {in }}{ }^{\text {ef }}$ e a temperatura nula, $p_{\text {ex }}>p_{\text {in }}$

\subsubsection{Temperatura Nula}

Para estimar a importância dos efeitos relativísticos, na Figura 4.17 comparamos as soluções da equação (4.31) com as soluções de (4.38), usando a EOS do modelo de sacola do MIT em temperatura nula [46,72] e a mQCD para a fase de QGP e o modelo não linear de Walecka (NLWM) $[46,51,61,164]$ para a fase de gás de hádron. Estas figuras ilustram o que acontece durante os estágios iniciais de uma colisão nuclear em 
energias intermediárias. Se a densidade crítica é alcançada, a bolha da fase de quarks começa a crescer, como mostramos nas Figuras 4.17(a) e 4.17(b). Depois as bolhas de hádrons começam a se contrair, o que mostramos nas Figuras 4.17(c) e 4.17(d). Destas figuras vemos que os efeitos relativísticos são relativamente pequenos. Por completeza também mostramos (nas linhas grossas tracejadas) nas Figuras 4.17(c) e 4.17(d) os resultados analíticos obtidos com (4.40), reescrita como $R(t)$. Como esperado temos boa concordância entre a solução numérica e a analítica.

Em todos os casos considerados a velocidade inicial da superfície da bolha é pequena e cresce para valores que ainda são menores do que da velocidade do som, o que é compatível com um processo "adiabático". A diferença, ainda que pequena, entre o resultado relativístico e não relativístico aparece somente em tempos maiores, quando a diferença de pressão atua tempo suficiente para acelerar a fronteira da bolha até velocidades maiores. A solução não relativística não possui um limite superior para a velocidade, e por isso vemos inclinações maiores em tempos maiores. Está é a mesma conclusão encontrada na Ref. [160], na qual os autores utilizaram uma EOS politrópica e para os casos onde $p_{i n}<<p_{e x}$. Da Figura 4.17 podemos concluir que o colapso de uma bolha de gás de hádrons imersa em um meio de QGP acontece em $3.3 \mathrm{fm}$ no caso relativístico e em $3.0 \mathrm{fm}$ no caso não relativístico. Com uma equação de estado para o QGP que possui pressão maior, o colapso acontece mais cedo, em $2.5 \mathrm{fm}$ no caso relativístico e em $2.2 \mathrm{fm}$ no caso não relativístico.

Na Figura 4.18 apresentamos a solução numérica da equação (4.31) para o caso de uma bolha de QGP (MIT ou mQCD) em um meio de hádrons (NLWM). De cima para baixo na figura, as equações de estado possuem mais pressão e assim a expansão é mais rápida. Como a escala é a mesma podemos verificar esta diferença através do aumento da inclinação das curvas. Mudando a velocidade inicial da fronteira da bolha não observamos uma mudança significativa da trajetória de $R(t)$. Quando aumentamos o tamanho da bolha de $1 \mathrm{fm}$ (painel esquerdo da Figura 4.18) para $2 \mathrm{fm}$ (painel direito da Figura 4.18) percebemos que as bolhas maiores se expandem mais vagarosamente que as menores. Podemos concluir que a bolha de QGP leva pelo menos (ver Figura 


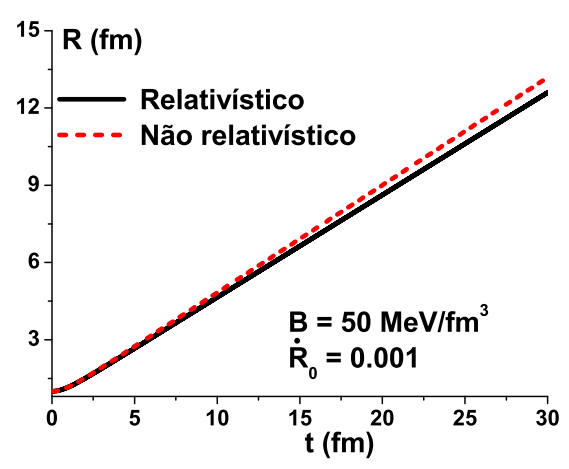

(a)

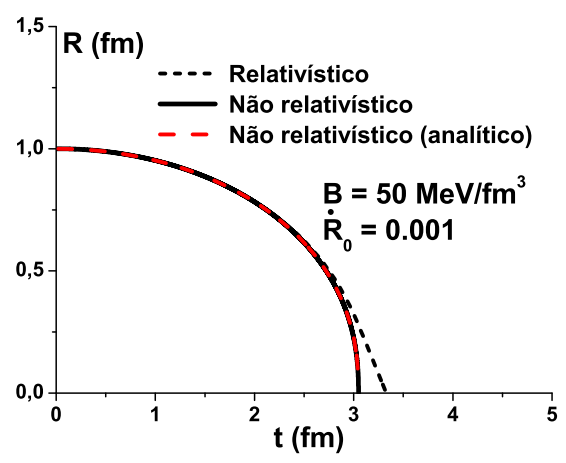

(c)

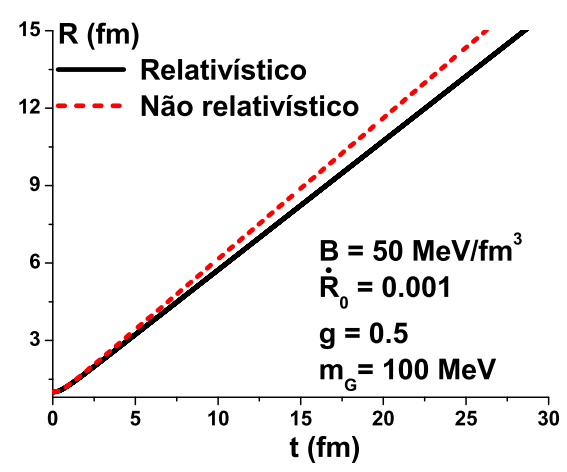

(b)

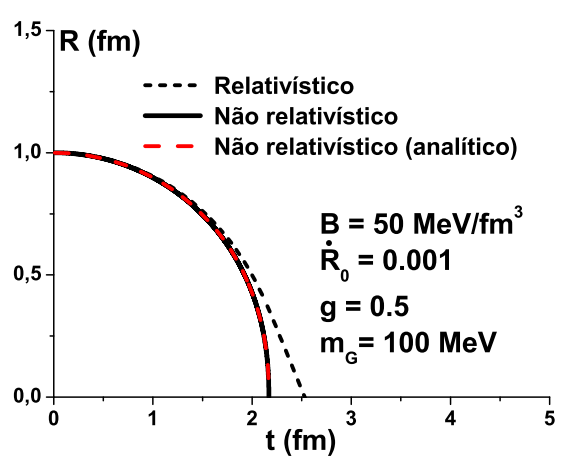

(d)

Figura 4.17: Comparação entre as soluções relativística e não relativística da equação $\boldsymbol{R P}$ com altas densidades $\left(\rho_{B}>\rho_{c}\right)$. a) e b) expansão da bolha de $\mathbf{Q G P}$ para diferentes EOS. c) e d) colapso da bolha de gás de hádrons para as mesmas duas EOS.

4.18(d) e 4.18(f)) $10 \mathrm{fm}$ para alcançar o tamanho de $\simeq 6 \mathrm{fm}$.

Na Figura 4.19 apresentamos a evolução temporal de uma bolha de gás de hádrons imersa no QGP. Esta figura é parecida com a Figura 4.18, com a diferença da fase "interna" e "externa". De cima para baixo na figura, a bolha se contrai mais rapidamente, mesmo quando a velocidade inicial está em sentido contrário. Como esperado, o colapso é mais rápido com a EOS que gera pressões maiores. 


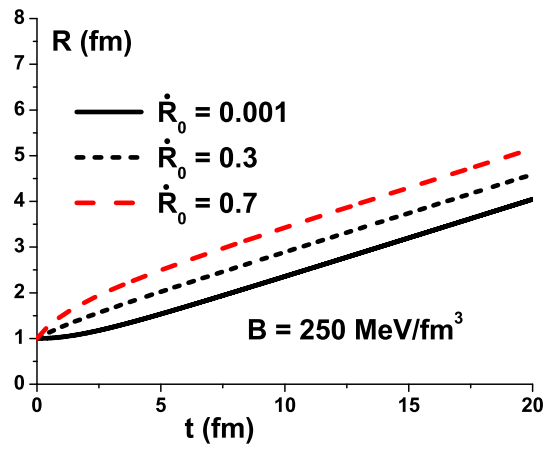

(a)

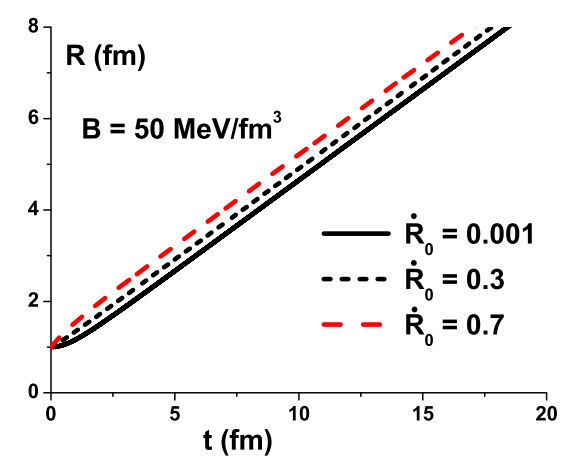

(c)

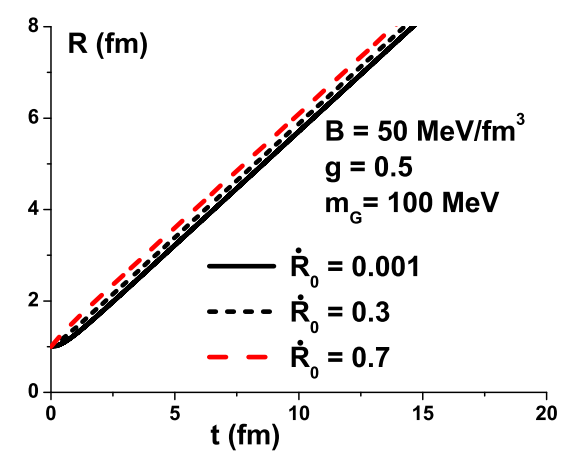

(e)

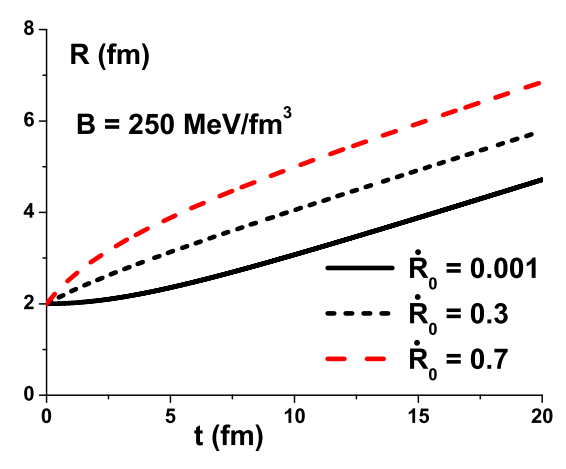

(b)

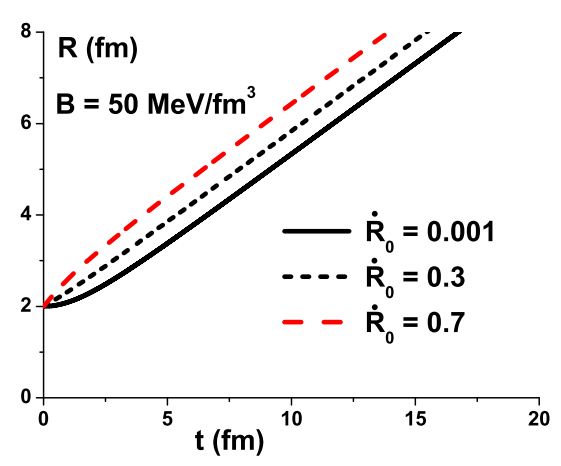

(d)

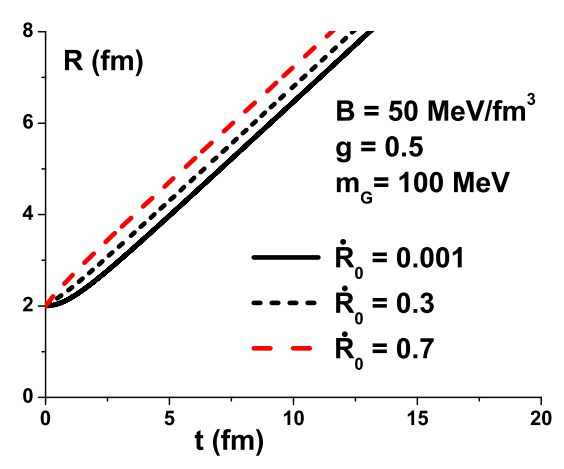

(f)

Figura 4.18: Tempo de evolução de uma bolha de QGP imersa em um gás de hádron. Soluções numéricas de (4.31) para diversas condições iniciais. Em todas situações: $\rho_{\text {ex }}=0.35 \mathrm{fm}^{-3}$ e $\rho_{\text {in }}=1.0 \mathrm{fm}^{-3}$. 


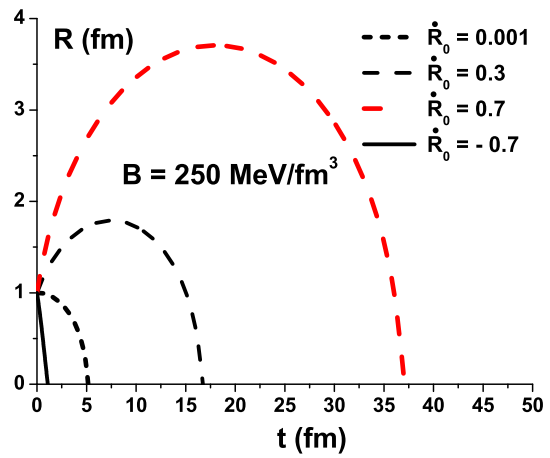

(a)

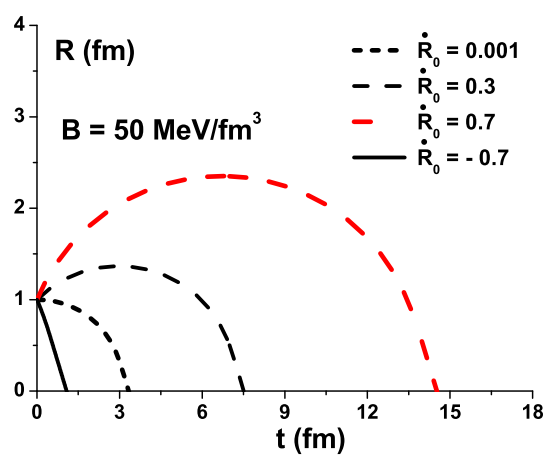

(c)

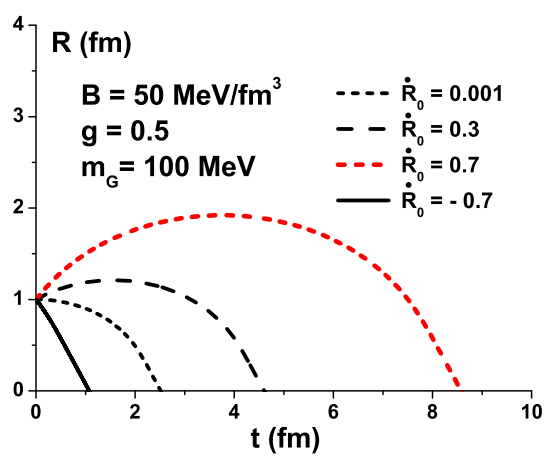

(e)

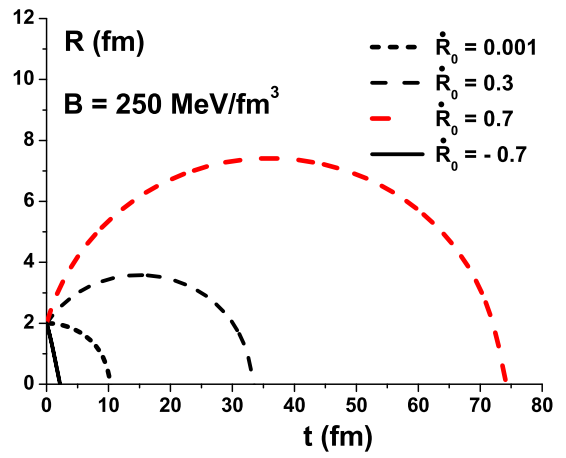

(b)

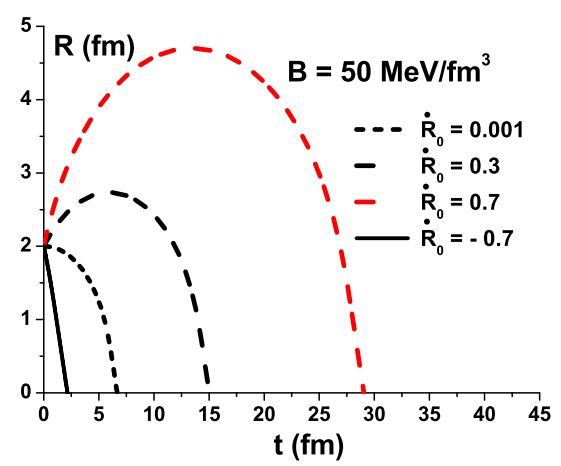

(d)

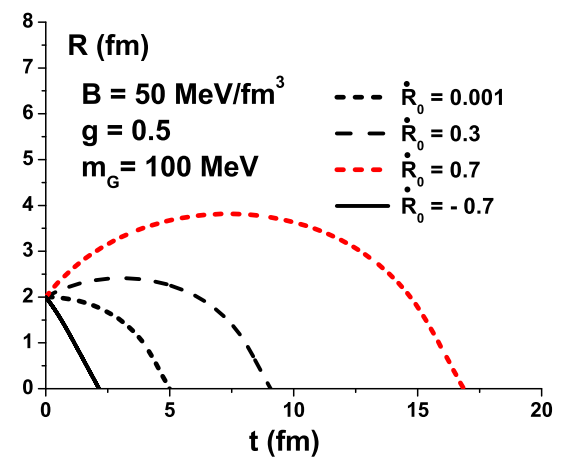

(f)

Figura 4.19: Tempo de evolução de uma bolha de gás de hádrons imerso no QGP. Soluções numéricas de (4.31) para diversas condições iniciais. Em todas situações: $\rho_{e x}=0.6 \mathrm{fm}^{-3}$ e $\rho_{i n}=0.4 \mathrm{fm}^{-3}$. 


\subsubsection{Temperatura Finita}

Na Figura 4.20 mostramos o caso com potencial químico grande: $\mu=350 \mathrm{MeV}$. As Figuras 4.20(a) e 4.20(b) mostram uma bolha de QGP (com EOS da QCD na rede) que se expande em um meio de gás de hádrons (NLWM), pois $p_{i n}{ }^{\text {ef }}>p_{e x}{ }^{\text {ef }}$, e as Figuras 4.20(c) e 4.20(d) mostram uma bolha de gás de hádrons que colapsa em um meio de QGP, pois $p_{i n}^{e f}<p_{e x}^{e f}$.

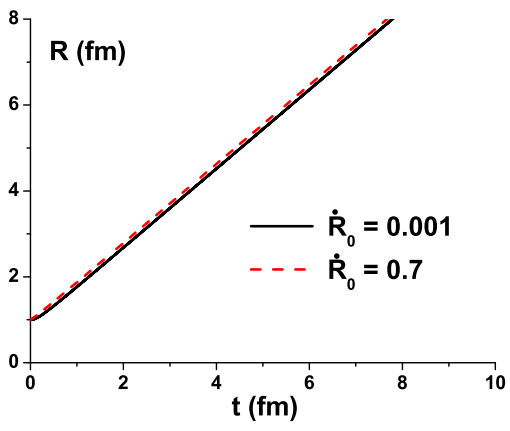

(a)

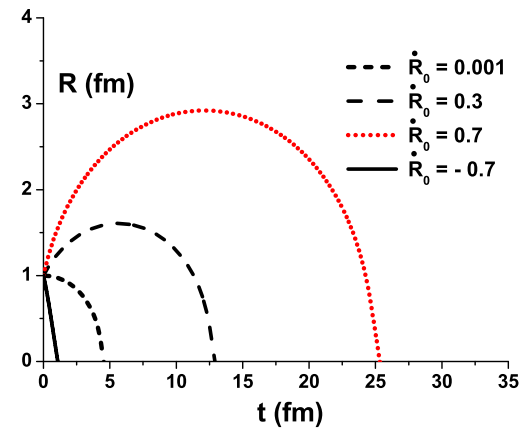

(c)

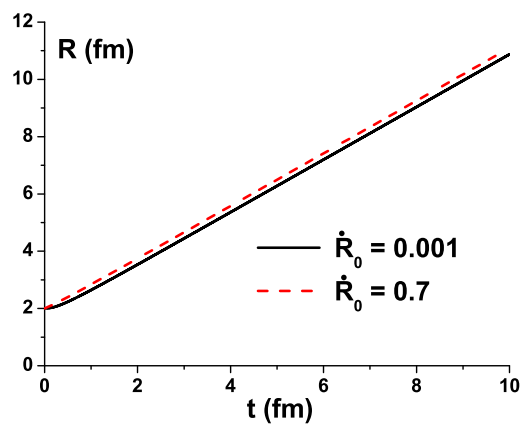

(b)

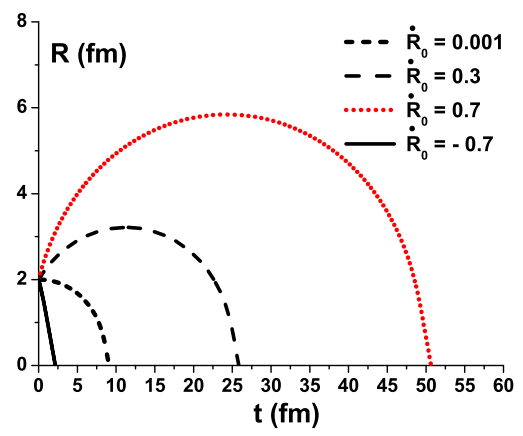

(d)

Figura 4.20: Tempo de evolução de uma bolha dada pela solução numérica de (4.31). Bolha de QGP com um raio inicial de $1 \mathrm{fm}$ (a) e $2 \mathrm{fm}$ (b) expandindo em um meio de gás de hádrons $\left(T_{e x}=140 \mathrm{MeV}\right.$ e $\left.T_{i n}=120 \mathrm{MeV}\right)$. Bolha de gás de hádrons de raio $1 \mathrm{fm}$ (c) e $2 \mathrm{fm}$ (d), em um meio de QGP ( $T_{e x}=120 \mathrm{MeV}$ e

$$
\left.T_{\text {in }}=138 \mathrm{MeV}\right) \text {. }
$$

Comparando as Figuras 4.20 e 4.18 podemos concluir que em temperatura finita, 
uma bolha de QGP em um meio de hádrons se expande mais rápido do que em temperatura nula. Porém, mesmo neste caso, o tempo necessário para uma bolha crescer e atingir um raio comparável ao tamanho do sistema $(R \simeq 6 \mathrm{fm})$ ainda é grande $(t \simeq 6$ $f m)$.

Nesta seção estendemos o formalismo variacional para dedução da RRP em temperatura finita e resolvemos a RRP com os efeitos de temperatura. Estimamos o tempo de expansão de uma bolha de QGP imersa num gás de hádrons resolvendo numericamente a equação de Rayleigh-Plesset relativística (RRP). As soluções da RRP foram obtidas com equações de estado mais modernas, o que exigiu certas aproximações, tais como a hipótese de que a densidade de energia e pressão não dependem da coordenada radial da bolha. Tais soluções mostram que o tempo de expansão é da ordem alguns fermis ou mais, o que deve ser levado em conta nas simulações de colisões em energias intermediárias que serão realizadas no FAIR e NICA.

Durante a expansão do sistema pode ser que uma bolha de QGP sobreviva como um domínio super-resfriado, que eventualmente pode emitir hádrons. A dinâmica de uma bolha de QGP super-resfriado foi estudada em detalhes em [156] e os efeitos do potencial químico foram discutido em [157].

Ao longo da evolução do fluido pode ocorrer o fenômeno da cavitação: a formação de bolhas de hádrons em um QGP ainda quente, constituindo um domínio de hádrons em altas temperaturas. Se a cavitação no QGP de fato ocorrer $[47,165,166]$, como consequência da viscosidade volumétrica, vamos observar uma queda de pressão no QGP e a formação de uma bolha hádrons. Esta bolha de hádrons sofre então pressão do QGP ao redor e, dependendo da diferença de pressão entre as duas fases, ela vai sofrer expansão ou colapso [47]. 


\subsection{Conclusão}

Este capítulo foi dedicado a mostrar algumas aplicações possíveis das equações de estado do QGP, em especial a EOS deduzida no Capítulo 2, mQCD. Vimos que a partir de ingredientes microscópicos podemos entender propriedades de objetos macroscópicos, como mostram os resultados obtidos no caso das estrelas compactas. Também podemos aplicar estes conceitos em cosmologia e em colisões de íons pesados relativísticos. 


\section{Capítulo 5}

\section{Conclusões, Críticas e Perspectivas}

Neste trabalho estudamos as mais recentes equações de estado do plasma de quarks e glúons e seus efeitos em astrofísica, cosmologia e colisões de íons pesados. Demos atenção à equação de estado chamada de mQCD, que foi desenvolvida pelo nosso grupo.

Após a introdução feita no primeiro capítulo, apresentamos no segundo capítulo a dedução geral da mQCD e seus limites nos casos particulares de temperatura nula e finita e potencial químico nulo e finito. Com esta equação de estado estudamos também os efeitos do campo magnético no QGP. Também utilizamos outras duas equações de estado: a do modelo de sacola do MIT e a da QCD na rede.

No capítulo três, verificamos como ondas se propagam neste plasma, com ênfase nos resultados obtidos com a mQCD e com campo magnético. Esta equação de estado gera ondas lineares que respeitam as condições de estabilidade e causalidade. A seguir derivamos a equação diferencial que descreve a propagação de ondas não lineares e encontramos (num contexto muito diferente daquele em que ela foi originalmente deduzida) a equação reduzida de Ostrovsky. Obtivemos uma solução analítica desta equação.

No capítulo 4 fizemos algumas aplicações das equações de estado discutidas anteriormente. Resolvendo a equação de Tolman-Oppenheimer-Volkoff, conseguimos obter 
resultados para as massas e raios de estrelas compactas, que estão em acordo com as predições e medidas realizadas. Indo até o limite deste formalismo, estudamos o papel do campo magnético na estabilidade das estrelas compactas. Resolvendo as equações de Friedmann estudamos a evolução temporal da densidade de energia e da temperatura na fase de QGP do Universo primordial. Concluímos que todas as equações de estado estudadas levam a resultados semelhantes. A seguir estudamos a evolução temporal de bolhas de QGP no gás de hádrons (e também de bolhas de gás de hádrons no QGP) produzido em colisões nucleares a energias intermediárias, como as que serão realizadas nos experimentos FAIR e NICA. Derivamos, pela primeira vez, a equação de Rayleigh-Plesset relativística (que descreve a evolução temporal de uma bolha) a temperatura finita. Usando a transformação de Sundman, encontramos uma solução analítica desta equação em um caso particular e determinamos o tempo de colapso das bolhas.

De um modo geral, os resultados relatados nesta tese são bastante sugestivos. No entando, por causa de aproximações feitas em vários pontos do trabalho, eles não podem ser considerados definitivos. Eles são suficientemente interessantes para justificar a continuação da pesquisa. Há várias deficiências que podemos corrigir. Em primeiro lugar, ao derivar a mQCD, podemos refinar a separação do campo de glúons, introduzindo uma escala de energia de separação e impondo que os resultados não dependam fortemente desta escolha. Isto pode ser feito com as técnicas do grupo de renormalização. Os parâmetros que entram na mQCD foram ajustados primeiro de maneira que a pressão fosse suficientemente grande para que existissem estrelas de nêutrons com duas massas solares. No entanto, estes parâmetros deveriam ter sido ajustados anteriormente, de modo a satisfazer os limites que já existem e que são impostos por teorias efetivas quirais, por QCD perturbativa e pelos resultados experimentais de ondas gravitacionais. Nós não fizemos isto em parte por razões históricas, pois o trabalho já tinha sido em boa parte executado quando surgiram os novos resultados teóricos e experimentais. Além disso, como a mQCD se propõe a ser mais geral e descrever o QGP em temperaturas baixas e altas e potenciais químicos 
baixos e altos, nós deveríamos também usar as equações de estado da QCD na rede, escolhendo os parâmetros da mQCD de forma que ela reproduza os resultados da LQCD.

O estudo de estrelas deveria ser refeito a partir das equações de Einstein com campo magnético. Além disso deveríamos fazer uso de modelos híbridos, nos quais além de matéria de quarks desconfinados, a estrela possui uma fase hadrônica. O estudo de cosmologia poderia ser refinado com a introdução de efeitos da viscosidade e com o estudo de ondas gravitacionais geradas no Universo primordial. Finalmente, no estudo da evolução de bolhas, deveríamos incluir os efeitos que um meio externo finito pode gerar e também levar em conta a dependência da densidade de energia e da pressão com o raio da bolha e ainda incluir o efeito da tensão superficial. 


\section{Apêndice A}

\section{Constantes de Estrutura de SU(3)}

Os geradores do grupo $\mathrm{SU}(3)$ são dados por:

$$
T^{a}=\frac{\lambda^{a}}{2} \quad a=1,2, \ldots, 8
$$

onde " $\lambda$ " são as matrizes de Gell-Mann dadas por:

$$
\begin{gathered}
\lambda^{1}=\left(\begin{array}{lll}
0 & 1 & 0 \\
1 & 0 & 0 \\
0 & 0 & 0
\end{array}\right) \quad \lambda^{2}=\left(\begin{array}{ccc}
0 & -i & 0 \\
i & 0 & 0 \\
0 & 0 & 0
\end{array}\right) \quad \lambda^{3}=\left(\begin{array}{ccc}
1 & 0 & 0 \\
0 & -1 & 0 \\
0 & 0 & 0
\end{array}\right) \\
\lambda^{4}=\left(\begin{array}{lll}
0 & 0 & 1 \\
0 & 0 & 0 \\
1 & 0 & 0
\end{array}\right) \\
\lambda^{7}=\left(\begin{array}{ccc}
0 & = \\
0 & 0 & -i \\
0 & i & 0
\end{array}\right)\left(\begin{array}{ccc}
0 & 0 & -i \\
0 & 0 & 0 \\
i & 0 & 0
\end{array}\right) \quad \lambda^{6}=\left(\begin{array}{lll}
0 & 0 & 0 \\
0 & 0 & 1 \\
0 & 1 & 0
\end{array}\right) \\
\lambda^{8}=\frac{1}{\sqrt{3}}\left(\begin{array}{ccc}
1 & 0 \\
0 & 1 & 0 \\
0 & 0 & -2
\end{array}\right)
\end{gathered}
$$


Os comutadores das matrizes são dados por:

$$
\left[\lambda^{a}, \lambda^{b}\right]=2 i f^{a b c} \lambda^{c}
$$

e $f^{a b c}$ sendo as constantes de estrutura anti-simétricas de SU(3), onde os índices de cor $a, b$ e $c$ variam de 1 a 8 . Também possuem a propriedade de anti-simetria:

$$
f^{b a c}=f^{a c b}=-f^{a b c}
$$

e as combinações não nulas, são:

$$
\begin{array}{r}
f^{123}=1 \\
f^{458}=f^{678}=\sqrt{3} / 2 \\
f^{147}=f^{246}=f^{257}=f^{345}=f^{516}=f^{637}=1 / 2
\end{array}
$$

Possuem relações importantes em $\mathrm{SU}(\mathrm{N})$ :

$$
\begin{array}{r}
f^{a b c} f^{a b c}=N\left(N^{2}-1\right) \stackrel{(\mathrm{N}=3)}{\longrightarrow} f^{a b c} f^{a b c}=24 \\
f^{a c d} f^{b c d}=N \delta^{a b} \stackrel{(\mathrm{N}=3)}{\longrightarrow} f^{a c d} f^{b c d}=3 \delta^{a b}
\end{array}
$$




\section{Apêndice B}

\section{Campo Fermiônico em}

\section{Temperatura Finita}

Em temperatura finita temos partículas e anti-partículas devido ao mar de Fermi. Os cálculos deste apêndice foram realizados como em [56, 61, 62].

A função de partição $Z$ pode ser escrita como:

$$
\begin{gathered}
Z=\operatorname{Tr}\left\{\exp \left[-\left(\hat{H}-\mu_{e} \hat{N}_{e}-\sum_{f=u}^{d, s} \mu_{f} \hat{N}_{f}\right) / T\right]\right\}= \\
\sum_{n_{1}, n_{2}, \ldots, n_{\infty}}\left\langle n_{1}, n_{2}, \ldots, n_{\infty}\left|\exp \left[-\left(\hat{H}-\mu_{e} \hat{N}_{e}-\sum_{f=u}^{d, s} \mu_{f} \hat{N}_{f}\right) / T\right]\right| n_{1}, n_{2}, \ldots, n_{\infty}\right\rangle
\end{gathered}
$$

Como os estados são autoestados dos operadores, podemos substituí-los pelos seus autovalores, ficando com:

$$
\begin{gathered}
Z=\sum_{n_{1}, n_{2}, \ldots, n_{\infty}}\left\langle n_{1}, n_{2}, \ldots, n_{\infty}\right| \exp \left[\left(-\sum_{i=1}^{\infty} E_{i} n_{i}+\mu_{e} \sum_{i=1}^{\infty} n_{i}\right.\right. \\
\left.\left.+\sum_{f=u}^{d, s} \mu_{f} \sum_{i=1}^{\infty} n_{i}\right) / T\right]\left|n_{1}, n_{2}, \ldots, n_{\infty}\right\rangle
\end{gathered}
$$




$$
\begin{gathered}
=\sum_{n_{1}}\left\langle n_{1}\left|\exp \left[\left(-E_{1} n_{1}+\mu_{e} n_{1}+\sum_{f=u}^{d, s} \mu_{f} n_{1}\right) / T\right]\right| n_{1}\right\rangle \times \\
\times \sum_{n_{2}}\left\langle n_{2}\left|\exp \left[\left(-E_{2} n_{2}+\mu_{e} n_{2}+\sum_{f=u}^{d, s} \mu_{f} n_{2}\right) / T\right]\right| n_{2}\right\rangle \ldots \\
\cdots \sum_{n_{\infty}}\left\langle n_{\infty}\left|\exp \left[\left(-E_{\infty} n_{\infty}+\mu_{e} n_{\infty}+\sum_{f=u}^{d, s} \mu_{f} n_{\infty}\right) / T\right]\right| n_{\infty}\right\rangle \\
=\prod_{i=1}^{\infty} \operatorname{Tr}_{i}\left\{\exp \left[\left(\mu_{e}+\sum_{f=u}^{d, s} \mu_{f}-E_{i}\right) n_{i} / T\right]\right\}
\end{gathered}
$$

Para os férmions, os números de ocupação podem ser 0 e 1, devido as suas relações de anticomutação, então a soma em (B.2) é restrita a esses valores:

$$
\begin{aligned}
Z & =\prod_{i=1}^{\infty} \sum_{n=0}^{1}\left\{\exp \left[\left(\mu_{e}+\sum_{f=u}^{d, s} \mu_{f}-E_{i}\right) n / T\right]\right\} \\
& =\prod_{i=1}^{\infty}\left\{1+\exp \left[\left(\mu_{e}+\sum_{f=u}^{d, s} \mu_{f}-E_{i}\right) / T\right]\right\}
\end{aligned}
$$

Os operadores $\hat{N}_{e}, \hat{N}_{f}$ e $\hat{H}$ são dados por [61]:

$$
\hat{N}_{e}=\hat{\mathcal{N}}_{e}-\left\langle 0\left|\hat{\mathcal{N}}_{e}\right| 0\right\rangle \quad, \quad \hat{N}_{f}=\hat{\mathcal{N}}_{f}-\left\langle 0\left|\hat{\mathcal{N}}_{f}\right| 0\right\rangle \quad \text { e } \quad \hat{H}=\hat{\mathcal{H}}-\langle 0|\hat{\mathcal{H}}| 0\rangle
$$

lembrando que $f=u, d, s$.

Em (B.4), temos que calcular os operadores:

$$
\begin{aligned}
\hat{\mathcal{N}}_{e} & =\int d^{3} x \bar{\psi}^{e} \gamma^{0} \psi^{e} \\
\hat{\mathcal{N}}_{f} & =\int d^{3} x \bar{\psi}^{f} \gamma^{0} \psi^{f}
\end{aligned}
$$

e

$$
\hat{\mathcal{H}}=\int d^{3} x\left\{\sum_{f=u}^{d, s} \frac{\partial \mathcal{L}_{0}}{\partial\left(\partial_{0} \psi^{f}\right)}\left(\partial^{0} \psi^{f}\right)+\frac{\partial \mathcal{L}_{0}}{\partial\left(\partial_{0} \psi^{e}\right)}\left(\partial^{0} \psi^{e}\right)-g^{00} \mathcal{L}_{0}\right\}
$$


Os campos dos férmions em termos de operadores são escritos como:

$$
\begin{aligned}
\psi_{i}^{f}= & c_{i} \frac{1}{\sqrt{V}} \sum_{\vec{k}, s, n}\left[A_{\vec{k}, s, n}^{f} \mathcal{U}^{f}(\vec{k}, s, n) e^{i \vec{k} \cdot \vec{x}-i E_{n}^{f(+)} t}\right. \\
& \left.+B^{\dagger f}{ }_{\vec{k}, s, n} \mathcal{V}^{f}(\vec{k}, s, n) e^{-i \vec{k} \cdot \vec{x}-i E_{n}^{f(-)} t}\right]
\end{aligned}
$$

onde $c_{i}$ são as matrizes de cor. Para o elétron:

$$
\begin{aligned}
\psi^{e}= & \frac{1}{\sqrt{V}} \sum_{\vec{k}, s, n}\left[A_{\vec{k}, s, n}^{e} \mathcal{U}^{e}(\vec{k}, s, n) e^{i \vec{k} \cdot \vec{x}-i E_{n}^{e(+)} t}\right. \\
& \left.+B^{\dagger e}{ }_{\vec{k}, s, n} \mathcal{V}^{e}(\vec{k}, s, n) e^{-i \vec{k} \cdot \vec{x}-i E_{n}^{e(-)} t}\right]
\end{aligned}
$$

onde os espinores satisfazem:

$$
\mathcal{U}^{\dagger^{i}}\left(\vec{k}^{\prime}, s^{\prime}, n^{\prime}\right) \mathcal{U}^{i}(\vec{k}, s, n)=\mathcal{V}^{\dagger^{i}}\left(\vec{k}^{\prime}, s^{\prime}, n^{\prime}\right) \mathcal{V}^{i}(\vec{k}, s, n)=\delta_{\vec{k}^{\prime} \vec{k}} \delta_{s^{\prime} s} \delta_{n^{\prime} n}
$$

e

$$
\mathcal{U}^{\dagger^{i}}\left(\vec{k}^{\prime}, s^{\prime}, n^{\prime}\right) \mathcal{V}^{i}(\vec{k}, s, n)=\mathcal{V}^{\dagger^{i}}\left(\vec{k}^{\prime}, s^{\prime}, n^{\prime}\right) \mathcal{U}^{i}(\vec{k}, s, n)=0
$$

Os operadores de criação e aniquilação:

$$
\left\{A^{i}, A^{\dagger^{i}}\right\}=\left\{B^{i}, B^{\dagger^{i}}\right\}=1 \quad e \quad\left\{A^{i}, B^{i}\right\}=\left\{A^{\dagger^{i}}, B^{\dagger^{i}}\right\}=0
$$

de (B.10) a (B.12) temos $i=e$ e $i=f=u, d, s$.

Substituindo (B.9) e (B.11) em (B.5):

$$
\begin{gathered}
\hat{\mathcal{N}}_{e}=\frac{1}{V} \int d^{3} x \sum_{\vec{k}^{\prime}, s^{\prime}, n^{\prime}} \sum_{\vec{k}, s, n}\left\{A_{\vec{k}^{\prime}, s^{\prime}, n^{\prime}}^{\dagger e} \mathcal{U}^{\dagger e}\left(\vec{k}^{\prime}, s^{\prime}, n^{\prime}\right) A_{\vec{k}, s, n}^{e} \mathcal{U}^{e}(\vec{k}, s, n) e^{-i\left(\vec{k}^{\prime}-\vec{k}\right) \cdot \vec{x}} \times\right. \\
\left.e^{i\left(E_{n^{\prime}}^{e(+)}-E_{n}^{e(+)}\right) t}+B_{\vec{k}^{\prime}, s^{\prime}, n^{\prime}}^{e} \mathcal{V}^{\dagger e}\left(\vec{k}^{\prime}, s^{\prime}, n^{\prime}\right) B_{\vec{k}, s, n}^{\dagger e} \mathcal{V}^{e}(\vec{k}, s, n) e^{i\left(\vec{k}^{\prime}-\vec{k}\right) \cdot \vec{x}} e^{i\left(E_{n^{\prime}}^{e(-)}-E_{n}^{e(-)}\right) t}\right\}
\end{gathered}
$$

e usando (B.10) e aplicando o ordenamento normal dos operadores de anti-partículas, 
ou seja, usar (B.12), chegamos em:

$$
\hat{\mathcal{N}}_{e}=\frac{1}{V} \int d^{3} x \sum_{\vec{k}, s, n}\left\{A_{\vec{k}, s, n}^{\dagger e} A_{\vec{k}, s, n}^{e}-B_{\vec{k}, s, n}^{\dagger e} B_{\vec{k}, s, n}^{e}+1\right\}
$$

Calculando o primeiro termo de (B.4) usando (B.13):

$$
\begin{gathered}
\hat{N}_{e}=\frac{1}{V} \int d^{3} x \sum_{\vec{k}, s, n}\left\{A_{\vec{k}, s, n}^{\dagger e} A_{\vec{k}, s, n}^{e}-B_{\vec{k}, s, n}^{\dagger e} B_{\vec{k}, s, n}^{e}\right\}+\frac{1}{V} \int d^{3} x \sum_{\vec{k}, s, n} 1 \\
-\left\langle 0\left|\frac{1}{V} \int d^{3} x \sum_{\vec{k}, s, n} A_{\vec{k}, s, n}^{\dagger e} A_{\vec{k}, s, n}^{e}\right| 0\right\rangle+\left\langle 0\left|\frac{1}{V} \int d^{3} x \sum_{\vec{k}, s, n} B_{\vec{k}, s, n}^{\dagger e} B_{\vec{k}, s, n}^{e}\right| 0\right\rangle \\
-\left\langle 0\left|\frac{1}{V} \int d^{3} x \sum_{\vec{k}, s, n} 1\right| 0\right\rangle
\end{gathered}
$$

resultando em:

$$
\hat{N}_{e}=\frac{1}{V} \int d^{3} x \sum_{\vec{k}, s, n}\left\{A_{\vec{k}, s, n}^{\dagger e} A_{\vec{k}, s, n}^{e}-B_{\vec{k}, s, n}^{\dagger e} B_{\vec{k}, s, n}^{e}\right\}
$$

Analogamente para os quarks, efetuando a somatória em $i$ e $j$ :

$$
c_{i}^{\dagger} \delta_{i j} c_{j}=c_{1}^{\dagger} c_{1}+c_{2}^{\dagger} c_{2}+c_{3}^{\dagger} c_{3}=3
$$

Porém, irá ser usado a média nas cores desse cálculo, ou seja, trocar o produto $c_{i}^{\dagger} \delta_{i j} c_{j}$ por sua "média nas cores" da seguinte forma:

$$
c_{i}^{\dagger} \delta_{i j} c_{j} \rightarrow \frac{c_{i}^{\dagger} \delta_{i j} c_{j}}{\text { (número de cores dos quarks) }}=\frac{c_{1}^{\dagger} c_{1}+c_{2}^{\dagger} c_{2}+c_{3}^{\dagger} c_{3}}{3}=1
$$

para que se possa definir a distribuição de Fermi-Dirac de forma usual. A cor dos quarks será levada em conta no fator estatístico. Sendo assim, o segundo termo de 
(B.4) possui a forma:

$$
\hat{N}_{f}=\frac{1}{V} \int d^{3} x \sum_{\vec{k}, s, n}\left\{A_{\vec{k}, s, n}^{\dagger f} A_{\vec{k}, s, n}^{f}-B_{\vec{k}, s, n}^{\dagger f} B_{\vec{k}, s, n}^{f}\right\}
$$

Para o cálculo do operador Hamiltoniano a partir do último termo de (B.4) e (B.7), é necessário alguns resultados. Fazendo $\bar{\psi}^{f} \times(2.22)$ com o uso de (B.8) e (B.11):

$$
\begin{gathered}
\frac{1}{V} \sum_{\vec{k}, s, n, \vec{k}^{\prime}, s^{\prime}, n^{\prime}}\left\{A_{\vec{k}^{\prime}, s^{\prime}, n^{\prime}}^{\dagger f} \mathcal{U}^{\dagger f}\left(\vec{k}^{\prime}, s^{\prime}, n^{\prime}\right) e^{-i \vec{k}^{\prime} \cdot \vec{x}+i E_{n^{\prime}}^{f(+)} t}\right. \\
\left.+B_{\vec{k}^{\prime}, s^{\prime}, n^{\prime}}^{f} \mathcal{V}^{\dagger f}\left(\vec{k}^{\prime}, s^{\prime}, n^{\prime}\right) e^{i \vec{k}^{\prime} \cdot \vec{x}+i E_{n^{\prime}}^{f(-)} t}\right\} c_{i}^{\dagger}\left[i \delta_{i j} \partial_{0}+i \delta_{i j} \vec{\alpha} \cdot \vec{\nabla}+g_{h} T_{i j}^{a} \alpha_{0}^{a}\right. \\
\left.-\delta_{i j} \gamma^{0}\left(m_{f}+Q_{f} \gamma^{\mu} A_{\mu}\right)\right] c_{j}\left\{A_{\vec{k}, s, n}^{f} \mathcal{U}^{f}(\vec{k}, s, n) e^{i \vec{k} \cdot \vec{x}-i E_{n}^{f(+)} t}\right. \\
\left.+B^{\dagger f}{ }_{\vec{k}, s, n} \mathcal{V}^{f}(\vec{k}, s, n) e^{-i \vec{k} \cdot \vec{x}-i E_{n}^{f(-)} t}\right\}=0
\end{gathered}
$$

que com (B.10) e (B.17):

$$
\begin{gathered}
\frac{1}{V} \sum_{\vec{k}, s, n}\left[E_{n}^{f(+)}-\vec{\alpha} \cdot \vec{k}+g_{h} \mathcal{A}-\gamma^{0}\left(m_{f}+Q_{f} \gamma^{\mu} A_{\mu}\right)\right] A_{\vec{k}, s, n}^{\dagger f} A_{\vec{k}, s, n}^{f} \\
+\frac{1}{V} \sum_{\vec{k}, s, n}\left[E_{n}^{f(-)}+\vec{\alpha} \cdot \vec{k}+g_{h} \mathcal{A}-\gamma^{0}\left(m_{f}+Q_{f} \gamma^{\mu} A_{\mu}\right)\right] B_{\vec{k}, s, n}^{f} B_{\vec{k}, s, n}^{\dagger f}=0
\end{gathered}
$$

sendo que $c_{i}^{\dagger} T_{i j}^{a} c_{j} \alpha_{0}^{a}=\mathcal{A}$.

Da expressão (B.19), as energias são:

$$
E_{n}^{f(+)}+g_{h} \mathcal{A}=\vec{\alpha} \cdot \vec{k}+\gamma^{0}\left(m_{f}+Q_{f} \gamma^{\mu} A_{\mu}\right)
$$

e

$$
E_{n}^{f(-)}+g_{h} \mathcal{A}=-\vec{\alpha} \cdot \vec{k}+\gamma^{0}\left(m_{f}+Q_{f} \gamma^{\mu} A_{\mu}\right)
$$


Usando (2.41) em (2.43):

$$
\tilde{E}_{n}^{f( \pm)} \equiv E_{n}^{f( \pm)}+g_{h} \mathcal{A}= \pm \sqrt{m_{f}^{2}+k_{z}^{2}+2 n\left|Q_{f}\right| B}
$$

Agora (B.20) e (B.21) com (B.22):

$$
\begin{aligned}
& \vec{\alpha} \cdot \vec{k}+\gamma^{0}\left(m_{f}+Q_{f} \gamma^{\mu} A_{\mu}\right) \longrightarrow \sqrt{m_{f}^{2}+k_{z}^{2}+2 n\left|Q_{f}\right| B} \\
& \vec{\alpha} \cdot \vec{k}-\gamma^{0}\left(m_{f}+Q_{f} \gamma^{\mu} A_{\mu}\right) \longrightarrow \sqrt{m_{f}^{2}+k_{z}^{2}+2 n\left|Q_{f}\right| B}
\end{aligned}
$$

Analogamente para os elétrons, sem a média nas cores conforme (B.17) e sem o termo de interação dos hard glúons:

$$
\begin{aligned}
& \vec{\alpha} \cdot \vec{k}+\gamma^{0}\left(m_{e}+Q_{e} \gamma^{\mu} A_{\mu}\right) \longrightarrow \sqrt{m_{e}^{2}+k_{z}^{2}+2 n\left|Q_{e}\right| B} \\
& \vec{\alpha} \cdot \vec{k}-\gamma^{0}\left(m_{e}+Q_{e} \gamma^{\mu} A_{\mu}\right) \longrightarrow \sqrt{m_{e}^{2}+k_{z}^{2}+2 n\left|Q_{e}\right| B}
\end{aligned}
$$

Voltando para o cálculo de (B.7), temos que:

$$
\begin{aligned}
\hat{\mathcal{H}}= & \int d^{3} x\left\{\sum_{f=u}^{d, s} i \psi_{i}^{\dagger} \delta_{i j}\left(\partial_{0} \psi_{j}^{f}\right)+i \psi_{i}^{\dagger} \delta_{i j}\left(\partial_{0} \psi_{j}^{e}\right)\right\}+\int d^{3} x\left\{-\frac{m_{G}{ }^{2}}{2} \alpha_{0}^{a} \alpha_{0}^{a}+b \phi_{0}^{4}+\frac{B^{2}}{8 \pi}\right\} \\
& +\int d^{3} x\left\{-\bar{\psi}_{i}^{e}\left[i \gamma^{\mu}\left(\delta_{i j} \partial_{\mu}+i \delta_{i j} Q_{e} A_{\mu}\right)-\delta_{i j} m_{e}\right] \psi_{j}^{e}\right\} \\
& +\int d^{3} x\left\{-\sum_{f=u}^{d, s} \bar{\psi}_{i}^{f}\left\{i \gamma^{\mu}\left[\delta_{i j} \partial_{\mu}+i \delta_{i j} Q_{f} A_{\mu}\right]+g_{h} \gamma^{0} T_{i j}^{a} \alpha_{0}^{a}-\delta_{i j} m_{f}\right\} \psi_{j}^{f}\right\}
\end{aligned}
$$

os termos com derivadas temporais dos campos dos férmions são algebricamente cancelados, ainda substituindo (B.8), (B.9), (B.17) e os resultados (B.23) à (B.26) em (B.27) e fazendo o ordenamento normal de (B.12), obtêm-se:

$$
\hat{\mathcal{H}}=\int d^{3} x\left\{-\frac{m_{G}{ }^{2}}{2} \alpha_{0}^{a} \alpha_{0}^{a}+b \phi_{0}{ }^{4}+\frac{B^{2}}{8 \pi}\right\}
$$




$$
\begin{gathered}
+\int d^{3} x\left\{\frac{1}{V} \sum_{\vec{k}, s, n} \sqrt{m_{e}^{2}+k_{z}^{2}+2 n\left|Q_{e}\right| B}\left[A_{\vec{k}, s, n}^{\dagger e} A_{\vec{k}, s, n}^{e}+B_{\vec{k}, s, n}^{\dagger e} B_{\vec{k}, s, n}^{e}\right]\right\} \\
-\int d^{3} x \frac{1}{V} \sum_{\vec{k}, s, n}\left(\sqrt{m_{e}^{2}+k_{z}^{2}+2 n\left|Q_{e}\right| B}\right) \\
+\int d^{3} x\left\{\frac{1}{V} \sum_{f=u}^{d, s} \sum_{\vec{k}, s, n} \sqrt{m_{f}^{2}+k_{z}^{2}+2 n\left|Q_{f}\right| B}\left[A_{\vec{k}, s, n}^{\dagger f} A_{\vec{k}, s, n}^{f}+B_{\vec{k}, s, n}^{\dagger f} B_{\vec{k}, s, n}^{f}\right]\right\} \\
-\int d^{3} x \frac{1}{V} \sum_{f=u}^{d, s} \sum_{\vec{k}, s, n}\left(\sqrt{m_{f}^{2}+k_{z}^{2}+2 n\left|Q_{f}\right| B}\right) \\
-\int d^{3} x\left\{\frac{1}{V} \sum_{f=u}^{d, s} \sum_{\vec{k}, s, n} g_{h}\left(c_{i}^{\dagger} T_{i j}^{a} c_{j}\right) \alpha_{0}^{a}\left[A_{\vec{k}, s, n}^{\dagger f} A_{\vec{k}, s, n}^{f}-B_{\vec{k}, s, n}^{\dagger f} B_{\vec{k}, s, n}^{f}\right]\right\} \\
-\int d^{3} x \frac{1}{V} \sum_{f=u}^{d, s} \sum_{\vec{k}, s, n}\left[g_{h}\left(c_{i}^{\dagger} T_{i j}^{a} c_{j}\right) \alpha_{0}^{a}\right]
\end{gathered}
$$

Assim, o operador Hamiltoniano é:

$$
\begin{gathered}
\hat{H}=\int d^{3} x\left\{-\frac{m_{G}^{2}}{2} \alpha_{0}^{a} \alpha_{0}^{a}+b \phi_{0}^{4}+\frac{B^{2}}{8 \pi}\right\} \\
+\int d^{3} x\left\{\frac{1}{V} \sum_{\vec{k}, s, n} \sqrt{m_{e}^{2}+k_{z}^{2}+2 n\left|Q_{e}\right| B}\left[A_{\vec{k}, s, n}^{\dagger e} A_{\vec{k}, s, n}^{e}+B_{\vec{k}, s, n}^{\dagger e} B_{\vec{k}, s, n}^{e}\right]\right\} \\
+\int d^{3} x\left\{\frac{3}{V} \sum_{f=u}^{d, s} \sum_{\vec{k}, s, n} \sqrt{m_{f}^{2}+k_{z}^{2}+2 n\left|Q_{f}\right| B}\left[A_{\vec{k}, s, n}^{\dagger f} A_{\vec{k}, s, n}^{f}+B_{\vec{k}, s, n}^{\dagger f} B_{\vec{k}, s, n}^{f}\right]\right\} \\
-\sum_{f=u}^{d, s} g_{h}\left(c_{i}^{\dagger} T_{i j}^{a} c_{j}\right) \alpha_{0}^{a} \hat{N}_{f}
\end{gathered}
$$

Voltando ao cálculo da função de partição. Substituindo (B.15), (B.18) e (B.29) 
em (B.1) e seguimos o raciocínio de (B.2) e (B.3):

$$
\begin{gathered}
Z=\exp \left[V\left\{-\frac{m_{G}{ }^{2}}{2} \alpha_{0}^{a} \alpha_{0}^{a}+b \phi_{0}{ }^{4}+\frac{B^{2}}{8 \pi}\right\} \frac{1}{T}\right] \times \\
\times \prod_{\vec{k}, s, n}\left\{1+\exp \left[-\left(\mathcal{E}_{n}^{e}-\mu_{e}\right) / T\right]\right\}\left\{1+\exp \left[-\left(\mathcal{E}_{n}^{e}+\mu_{e}\right) / T\right]\right\} \\
\times \prod_{f=u}^{d, s} \prod_{\vec{k}, s, n}\left\{1+\exp \left[-\left(\mathcal{E}_{n}^{f}-\nu_{f}\right) / T\right]\right\}\left\{1+\exp \left[-\left(\mathcal{E}_{n}^{f}+\nu_{f}\right) / T\right]\right\}
\end{gathered}
$$

onde as energias são dadas por:

$$
\begin{aligned}
& \mathcal{E}_{n}^{e}=\sqrt{m_{e}^{2}+k_{z}^{2}+2 n\left|Q_{e}\right| B} \\
& \mathcal{E}_{n}^{f}=\sqrt{m_{f}^{2}+k_{z}^{2}+2 n\left|Q_{f}\right| B}
\end{aligned}
$$

e o potencial químico efetivo do quark $f$ é:

$$
\nu_{f} \equiv \mu_{f}+g_{h}\left(c_{i}^{\dagger} T_{i j}^{a} c_{j}\right) \alpha_{0}^{a}
$$

O potencial termodinâmico, (2.50), com (B.30) é:

$$
\begin{gathered}
\Omega=\left[-\frac{m_{G}^{2}}{2} \alpha_{0}^{a} \alpha_{0}^{a}+b \phi_{0}^{4}+\frac{B^{2}}{8 \pi}\right] V \\
-T \sum_{\vec{k}, s, n}\left\{\ln \left[1+e^{-\left(\mathcal{E}_{n}^{e}-\mu_{e}\right) / T}\right]+\ln \left[1+e^{-\left(\mathcal{E}_{n}^{e}+\mu_{e}\right) / T}\right]\right\} \\
-T \sum_{f=u}^{d, s} \sum_{\vec{k}, s, n}\left\{\ln \left[1+e^{-\left(\mathcal{E}_{n}^{f}-\nu_{f}\right) / T}\right]+\ln \left[1+e^{-\left(\mathcal{E}_{n}^{f}+\nu_{f}\right) / T}\right]\right\}
\end{gathered}
$$

Das distribuições, destacam-se algumas relações importantes:

$$
1+e^{-\left(\mathcal{E}_{n}^{i}-\nu_{i}\right) / T}=\left(1-d_{i}\right)^{-1}
$$




$$
\begin{aligned}
1+e^{-\left(\mathcal{E}_{n}^{i}+\nu_{i}\right) / T} & =\left(1-\bar{d}_{i}\right)^{-1} \\
e^{\left(\mathcal{E}_{n}^{i}-\nu_{i}\right) / T} & =\frac{\left(1-d_{i}\right)}{d_{i}}
\end{aligned}
$$

e

$$
e^{\left(\mathcal{E}_{n}^{i}+\nu_{i}\right) / T}=\frac{\left(1-\bar{d}_{i}\right)}{\bar{d}_{i}}
$$




\section{Apêndice $\mathrm{C}$}

\section{Aproximações para Altas e Baixas}

\section{Temperaturas}

De (2.87), temos a integral:

$$
I_{p} \equiv \int_{0}^{\infty} d k k^{2}\left\{\ln \left[1+e^{-\left(\mathcal{E}_{i}-\nu_{i}\right) / T}\right]+\ln \left[1+e^{-\left(\mathcal{E}_{i}+\nu_{i}\right) / T}\right]\right\}
$$

Utilizando a expansão:

$$
\ln (1+\omega)=\sum_{n=1}^{\infty} \frac{(-1)^{n+1}}{n} \omega^{n} \quad \text { para }|\omega|<1
$$

(C.1), pode ser reescrita:

$$
I_{p}=-\int_{0}^{\infty} d k k^{2} \sum_{n=1}^{\infty} \frac{(-1)^{n}}{n} e^{-n\left(\mathcal{E}_{i}-\nu_{i}\right) / T}-\int_{0}^{\infty} d k k^{2} \sum_{n=1}^{\infty} \frac{(-1)^{n}}{n} e^{-n\left(\mathcal{E}_{i}+\nu_{i}\right) / T}
$$

Com as mudanças de variáveis:

$$
z=\frac{m_{i}}{T} \quad \text { e } \quad x=\frac{k}{m_{i}}
$$


(C.3) fica como:

$$
I_{p}=-T^{3} z^{3} \int_{0}^{\infty} d x x^{2} \sum_{n=1}^{\infty} \frac{(-1)^{n}}{n} e^{-n z \sqrt{1+x^{2}}}\left(e^{n \nu_{i} / T}+e^{-n \nu_{i} / T}\right)
$$

ou:

$$
I_{p}=T^{3} z^{3} \sum_{n=1}^{\infty} \frac{(-1)^{n}}{n^{2}}\left(e^{n \nu_{i} / T}+e^{-n \nu_{i} / T}\right) \frac{\partial}{\partial z} \int_{0}^{\infty} d x x^{2} \frac{e^{-n z \sqrt{1+x^{2}}}}{\sqrt{1+x^{2}}}
$$

Fazendo $x=\operatorname{senh}(y)$, (C.6) assume a forma:

$$
I_{p}=T^{3} z^{3} \sum_{n=1}^{\infty} \frac{(-1)^{n}}{n^{2}}\left(e^{n \nu_{i} / T}+e^{-n \nu_{i} / T}\right) \frac{\partial}{\partial z} \int_{0}^{\infty} d y \operatorname{senh}^{2}(y) e^{-n z \cosh (y)}
$$

que remete a forma da função de Bessel modificada $K$ [68]:

$$
K_{\nu}[\omega]=\frac{\pi^{1 / 2}}{(\nu-1 / 2) !}\left(\frac{\omega}{2}\right)^{\nu} \int_{0}^{\infty} d y \operatorname{senh}^{2 \nu}(y) e^{-\omega \cosh (y)}
$$

(C.7) pode ser reescrita usando $(1 / 2) !=\pi^{1 / 2} / 2$ e fazendo o cálculo da derivada:

$$
I_{p}=T^{3} z^{3} \sum_{n=1}^{\infty} \frac{(-1)^{n}}{n^{2}}\left(e^{n \nu_{i} / T}+e^{-n \nu_{i} / T}\right)\left(-\frac{K_{1}[n z]}{n z^{2}}+\frac{K_{1}^{\prime}[n z]}{z}\right)
$$

Das relações de recorrência para as funções de Bessel [68]:

$$
K_{\nu-1}[\omega]-K_{\nu+1}[\omega]=-\frac{2 \nu}{w} K_{\nu}[\omega]
$$

e

$$
K_{\nu-1}[\omega]+K_{\nu+1}[\omega]=-2 K_{\nu}^{\prime}[\omega]
$$

se tem (C.9) como:

$$
I_{p}=-T^{3} z^{2} \sum_{n=1}^{\infty} \frac{(-1)^{n}}{n^{2}}\left(e^{n \nu_{i} / T}+e^{-n \nu_{i} / T}\right) K_{2}[n z]
$$


Da expressão da densidade de energia (2.90) temos a seguinte integral:

$$
I_{\varepsilon}=\int_{0}^{\infty} d k k^{2} \sqrt{m_{i}^{2}+k^{2}}\left(d_{i}+\bar{d}_{i}\right)
$$

que com o uso de (C.4) e:

$$
\left(1+e^{-z \sqrt{1+x^{2}}}\right)^{-1}=\sum_{n=0}^{\infty}(-1)^{n} e^{-n z \sqrt{1+x^{2}}}
$$

fica na forma:

$$
\begin{gathered}
I_{\varepsilon}=T^{4} \int_{0}^{\infty} d x x^{2} z^{4}\left(\sqrt{1+x^{2}}\right) \sum_{n=0}^{\infty}(-1)^{n}\left[e^{(n+1) \nu_{i} / T}+e^{-(n+1) \nu_{i} / T}\right] \\
\times e^{-(n+1) z \sqrt{1+x^{2}}}
\end{gathered}
$$

que ainda é reescrita como:

$$
\begin{gathered}
I_{\varepsilon}=T^{4} z^{4} \sum_{n=0}^{\infty} \frac{(-1)^{n}}{(n+1)^{2}}\left[e^{(n+1) \nu_{i} / T}+e^{-(n+1) \nu_{i} / T}\right] \\
\times \frac{\partial^{2}}{\partial z^{2}} \int_{0}^{\infty} d x \frac{x^{2}}{\sqrt{1+x^{2}}} e^{-(n+1) z \sqrt{1+x^{2}}}
\end{gathered}
$$

Novamente, $x=\operatorname{senh}(y)$ e a expressão (C.16) se torna:

$$
\begin{aligned}
I_{\varepsilon}= & T^{4} z^{4} \sum_{n=0}^{\infty} \frac{(-1)^{n}}{(n+1)^{2}}\left[e^{(n+1) \nu_{i} / T}+e^{-(n+1) \nu_{i} / T}\right] \\
& \times \frac{\partial^{2}}{\partial z^{2}} \int_{0}^{\infty} d y \operatorname{senh}^{2}(y) e^{-(n+1) z \cosh (y)}
\end{aligned}
$$

que com (C.8):

$$
I_{\varepsilon}=T^{4} z^{4} \sum_{n=0}^{\infty} \frac{(-1)^{n}}{(n+1)^{2}}\left[e^{(n+1) \nu_{i} / T}+e^{-(n+1) \nu_{i} / T}\right] \frac{2(1 / 2) !}{\pi^{1 / 2}} \frac{\partial^{2}}{\partial z^{2}}\left(\frac{K_{1}[(n+1) z]}{(n+1) z}\right)
$$


Fazendo os cálculos das derivadas com as relações de recorrência (C.10) e (C.11):

$$
\begin{gathered}
\frac{\partial^{2}}{\partial z^{2}}\left(\frac{K_{1}[(n+1) z]}{(n+1) z}\right)=\frac{\partial}{\partial z}\left\{-\frac{1}{(n+1) z^{2}}\left[K_{1}[(n+1) z]-(n+1) z K_{1}^{\prime}[(n+1) z]\right]\right\} \\
\frac{\partial}{\partial z}\left\{-\frac{K_{2}[(n+1) z]}{z}\right\}=\frac{3 K_{2}[(n+1) z]}{z^{2}}+\frac{(n+1) K_{1}[(n+1) z]}{z}
\end{gathered}
$$

Usando (1/2)! $=\pi^{1 / 2} / 2$ e (C.19) a expressão (C.18) assume a forma:

$$
\begin{aligned}
I_{\varepsilon} & =T^{4} z^{2} \sum_{n=0}^{\infty} \frac{(-1)^{n}}{(n+1)^{2}}\left[e^{(n+1) \nu_{i} / T}+e^{-(n+1) \nu_{i} / T}\right] \\
& \times\left\{3 K_{2}[(n+1) z]+(n+1) z K_{1}[(n+1) z]\right\}
\end{aligned}
$$

A integral da densidade de quarks e de elétrons (2.88) é dada por:

$$
I=\int_{0}^{\infty} d k k^{2}\left(d_{i}-\bar{d}_{i}\right)
$$

ou melhor:

$$
I=\int_{0}^{\infty} d k k^{2}\left\{\frac{e^{-\left(\mathcal{E}_{i}-\nu_{i}\right) / T}}{\left[1+e^{-\left(\mathcal{E}_{i}-\nu_{i}\right) / T}\right]}-\frac{e^{-\left(\mathcal{E}_{i}+\nu_{i}\right) / T}}{\left[1+e^{-\left(\mathcal{E}_{i}+\nu_{i}\right) / T}\right]}\right\}
$$

que novamente usando (C.4) e a expansão (C.14):

$$
I=m_{i}{ }^{3} \int_{0}^{\infty} d x x^{2} \sum_{n=0}^{\infty}(-1)^{n}\left[e^{(n+1) \nu_{i} / T}-e^{-(n+1) \nu_{i} / T}\right] e^{-(n+1) z \sqrt{1+x^{2}}}
$$

$\mathrm{Ou}$

$$
I=-m_{i}{ }^{3} \int_{0}^{\infty} d x x^{2} \sum_{n=0}^{\infty}(-1)^{n}\left[e^{(n+1) \nu_{i} / T}-e^{-(n+1) \nu_{i} / T}\right] \frac{\partial}{\partial z}\left\{\frac{e^{-(n+1) z \sqrt{1+x^{2}}}}{(n+1) \sqrt{1+x^{2}}}\right\}
$$

Novamente, $x=\operatorname{senh}(y)$, encontramos:

$$
I=-m_{i}{ }^{3} \sum_{n=0}^{\infty} \frac{(-1)^{n}}{(n+1)}\left[e^{(n+1) \nu_{i} / T}-e^{-(n+1) \nu_{i} / T}\right] \frac{\partial}{\partial z} \int_{0}^{\infty} \operatorname{senh}^{2}(y) e^{-(n+1) z \cosh (y)}
$$


com (C.8) e as relações de recorrência (C.10) e (C.11), (C.25):

$$
I=T^{3} z^{2} \sum_{n=0}^{\infty} \frac{(-1)^{n}}{(n+1)}\left[e^{(n+1) \nu_{i} / T}-e^{-(n+1) \nu_{i} / T}\right] K_{2}[(n+1) z]
$$

As expressões (C.12), (C.20) e (C.26) são válidas para potenciais químicos finitos apenas na aproximação $\sqrt{m_{i}^{2}+k^{2}}>\nu_{f}$.

\section{C.1 Altas temperaturas}

Para o caso de altas temperaturas $T>>\nu_{i}$ e $T>>m_{i}$ (ou $z<<1$ ) vale a forma assintótica para pequenos argumentos das funções de Bessel [68]:

$$
K_{\nu}[\omega]_{(\text {pequeno } \omega)}=\frac{(\nu-1) ! 2^{\nu-1}}{\omega^{\nu}}
$$

e também as expansões:

$$
\begin{gathered}
e^{n \nu_{i} / T}+e^{-n \nu_{i} / T} \cong 2+\frac{n^{2} \nu_{i}^{2}}{T^{2}} \\
e^{(n+1) \nu_{i} / T}+e^{-(n+1) \nu_{i} / T} \cong 2+\frac{(n+1)^{2} \nu_{i}^{2}}{T^{2}}
\end{gathered}
$$

$\mathrm{e}$

$$
e^{(n+1) \nu_{i} / T}-e^{-(n+1) \nu_{i} / T} \cong \frac{2(n+1) \nu_{i}}{T}
$$

Sendo assim, com (C.27) a (C.30), (C.20), (C.12) e (C.26) no limite de altas temperaturas:

$$
I_{\varepsilon(\text { T alta })}=T^{4} \sum_{n=0}^{\infty} \frac{(-1)^{n}}{(n+1)^{2}}\left\{\frac{12}{(n+1)^{2}}+2 z^{2}+\frac{6 \nu_{i}^{2}}{T^{2}}+\frac{(n+1)^{2} \nu_{i}^{2} z^{2}}{T^{2}}\right\}
$$


que desprezando os termos de $z^{2}$, pois $z<<1$ :

$$
\begin{aligned}
& I_{\varepsilon\left(T_{\text {alta })}\right.} \cong T^{4}\left[\sum_{n=0}^{\infty} 12 \frac{(-1)^{n}}{(n+1)^{4}}+\sum_{n=0}^{\infty} 6 \frac{(-1)^{n}}{(n+1)^{2}} \frac{\nu_{i}^{2}}{T^{2}}\right] \\
& I_{p_{(T \text { alta })}}=-2 T^{3}\left[\sum_{n=1}^{\infty} 2 \frac{(-1)^{n}}{n^{4}}+\sum_{n=1}^{\infty} \frac{(-1)^{n}}{n^{2}} \frac{\nu_{i}^{2}}{T^{2}}\right]
\end{aligned}
$$

e

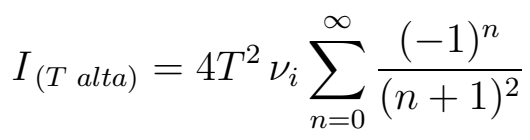

De [69]:

$$
\begin{aligned}
\sum_{n=0}^{\infty} \frac{(-1)^{n}}{(n+1)^{4}}=\frac{7 \pi^{4}}{720} & \text { e } & \sum_{n=0}^{\infty} \frac{(-1)^{n}}{(n+1)^{2}}=\frac{\pi^{2}}{12} \\
\sum_{n=1}^{\infty} \frac{(-1)^{n}}{n^{4}}=-\frac{7 \pi^{4}}{720} & \text { e } & \sum_{n=1}^{\infty} \frac{(-1)^{n}}{n^{2}}=-\frac{\pi^{2}}{12}
\end{aligned}
$$

Assim, (C.34) em (C.31):

$$
I_{\varepsilon(T \text { alta })}=\frac{7 \pi^{4}}{60} T^{4}+\frac{\pi^{2}}{2} T^{2} \nu_{i}^{2}
$$

(C.35) em (C.32):

$$
I_{p_{(T \text { alta })}}=\frac{7 \pi^{4}}{180} T^{3}+\frac{\pi^{2}}{6} T \nu_{i}^{2}
$$

(C.34) em (C.33):

$$
I_{(T \text { alta })}=\frac{\pi^{2}}{3} T^{2} \nu_{i}
$$

\section{C.2 Baixas temperaturas}

Para o caso de temperaturas baixas $T<<m_{i}$ (ou $z>>1$ ), a forma assintótica 
para grandes argumentos da função de Bessel [68] é:

$$
K_{\nu}[\omega]_{(\text {grande } \omega)}=\sqrt{\frac{\pi}{2 \omega}} e^{-\omega}\left[1+i \frac{\left(4 \nu^{2}-1\right)}{1 ! 8 i \omega}\right]=\sqrt{\frac{\pi}{2 \omega}} e^{-\omega}\left[1+\frac{\left(4 \nu^{2}-1\right)}{1 ! 8 \omega}\right]
$$

Substituindo então (C.39) em (C.12), para a pressão:

$$
I_{p_{(T \text { baixa })}}=-T^{3} z^{2} \sum_{n=1}^{\infty} \frac{(-1)^{n}}{n^{2}}\left(e^{n \nu_{i} / T}+e^{-n \nu_{i} / T}\right) \sqrt{\frac{\pi}{2 n z}} e^{-n z}\left(1+\frac{15}{8 n z}\right)
$$

que considerando apenas $n=1$ na somatória e também desprezando o termo 15/8z, pois $z>>1$ :

$$
I_{p_{(T \text { baixa })}}=T^{3} z^{3 / 2} \sqrt{\frac{\pi}{2}}\left[e^{-\left(z-\nu_{i} / T\right)}+e^{-\left(z+\nu_{i} / T\right)}\right]
$$

Com (C.4), (C.40):

$$
I_{p_{(T \text { baixa })}}=\left(m_{i} T\right)^{3 / 2} \sqrt{\frac{\pi}{2}}\left[e^{-\left(m_{i}-\nu_{i}\right) / T}+e^{-\left(m_{i}+\nu_{i}\right) / T}\right]
$$

Para a densidade de férmions, (C.39) em (C.26):

$$
\begin{aligned}
I= & T^{3} z^{2} \sum_{n=0}^{\infty} \frac{(-1)^{n}}{(n+1)}\left[e^{(n+1) \nu_{i} / T}-e^{-(n+1) \nu_{i} / T}\right] \\
& \times \sqrt{\frac{\pi}{2(n+1) z}} e^{-(n+1) z}\left[1+\frac{15}{8(n+1) z}\right]
\end{aligned}
$$

que com (C.4) e considerando só $n=0$ na somatória e desprezando o termo 15/8z, pois $z>>1$ :

$$
I_{(T \text { baixa })}=\left(m_{i} T\right)^{3 / 2} \sqrt{\frac{\pi}{2}}\left[e^{-\left(m_{i}-\nu_{i}\right) / T}-e^{-\left(m_{i}+\nu_{i}\right) / T}\right]
$$

Agora com (C.39) em (C.20) para a integral da densidade de energia:

$$
I_{\varepsilon(T \text { baixa })}=T^{4} z^{3 / 2} \sum_{n=0}^{\infty} \frac{(-1)^{n}}{(n+1)^{2}}\left[e^{-(n+1)\left(z-\nu_{i} / T\right)}+e^{-(n+1)\left(z+\nu_{i} / T\right)}\right] \sqrt{\frac{\pi}{2(n+1)}}
$$




$$
\times\left\{\frac{27}{8}+(n+1) z+\frac{45}{8(n+1) z}\right\}
$$

que considerando apenas $n=0$ na somatória e também desprezando o termo 45/8z, pois $z>>1$ :

$$
I_{\varepsilon(T \text { baixa })}=T^{4} z^{3 / 2}\left[e^{-\left(z-\nu_{i} / T\right)}+e^{-\left(z+\nu_{i} / T\right)}\right] \sqrt{\frac{\pi}{2}}\left(\frac{27}{8}+z\right)
$$

que com (C.4):

$$
I_{\varepsilon(T \text { baixa })}=T^{5 / 2} m_{i}^{3 / 2} \sqrt{\frac{\pi}{2}}\left(\frac{27}{8}+\frac{m_{i}}{T}\right)\left[e^{-\left(m_{i}-\nu_{i}\right) / T}+e^{-\left(m_{i}+\nu_{i}\right) / T}\right]
$$

Então as densidades dos férmions são:

$$
\rho_{f_{(T \text { baixa })}}=\sum_{f=u}^{d, s} \frac{\gamma_{f}}{\sqrt{8 \pi^{3}}}\left(m_{f} T\right)^{3 / 2}\left[e^{-\left(m_{f}-\nu_{f}\right) / T}-e^{-\left(m_{f}+\nu_{f}\right) / T}\right]
$$

e

$$
\rho_{e(T \text { baixa })}=\frac{\gamma_{e}}{\sqrt{8 \pi^{3}}}\left(m_{e} T\right)^{3 / 2}\left[e^{-\left(m_{e}-\mu_{e}\right) / T}-e^{-\left(m_{e}+\mu_{e}\right) / T}\right]
$$

A densidade de energia é:

$$
\begin{gathered}
\varepsilon_{(T \text { baixa })}=\frac{3 g_{h}^{2}}{2 N m_{G}^{2}}\left\{\sum_{f=u}^{d, s} \frac{\gamma_{f}}{\sqrt{8 \pi^{3}}}\left(m_{f} T\right)^{3 / 2}\left[e^{-\left(m_{f}-\nu_{f}\right) / T}-e^{-\left(m_{f}+\nu_{f}\right) / T}\right]\right\}^{2} \\
+b \phi_{0}^{4}+\frac{\gamma_{e}}{\sqrt{8 \pi^{3}}} T^{5 / 2} m_{e}^{3 / 2}\left(\frac{27}{8}+\frac{m_{e}}{T}\right)\left[e^{-\left(m_{e}-\mu_{e}\right) / T}+e^{-\left(m_{e}+\mu_{e}\right) / T}\right] \\
+\sum_{f=u}^{d, s} \frac{\gamma_{f}}{\sqrt{8 \pi^{3}}} T^{5 / 2} m_{f}^{3 / 2}\left(\frac{27}{8}+\frac{m_{f}}{T}\right)\left[e^{-\left(m_{f}-\nu_{f}\right) / T}+e^{-\left(m_{f}+\nu_{f}\right) / T}\right]
\end{gathered}
$$

e a pressão é escrita como:

$$
p_{(T \text { baixa })}=\frac{3 g_{h}^{2}}{2 N m_{G}^{2}}\left\{\sum_{f=u}^{d, s} \frac{\gamma_{f}}{\sqrt{8 \pi^{3}}}\left(m_{f} T\right)^{3 / 2}\left[e^{-\left(m_{f}-\nu_{f}\right) / T}-e^{-\left(m_{f}+\nu_{f}\right) / T}\right]\right\}^{2}
$$




$$
\begin{gathered}
-b \phi_{0}^{4}+\frac{\gamma_{e}}{\sqrt{8 \pi^{3}}} T^{5 / 2} m_{e}^{3 / 2}\left[e^{-\left(m_{e}-\mu_{e}\right) / T}-e^{-\left(m_{e}+\mu_{e}\right) / T}\right] \\
+\sum_{f=u}^{d, s} \frac{\gamma_{f}}{\sqrt{8 \pi^{3}}} T^{5 / 2} m_{f}^{3 / 2}\left[e^{-\left(m_{f}-\nu_{f}\right) / T}-e^{-\left(m_{f}+\nu_{f}\right) / T}\right]
\end{gathered}
$$




\section{Apêndice D}

\section{Solução Exata para a ROE}

O objetivo é encontrar a solução para a ROE:

$$
\frac{\partial}{\partial \xi}\left[\frac{\partial}{\partial t} \delta \rho_{B f}+\alpha \frac{\partial}{\partial \xi} \delta \rho_{B f}+\beta \delta \rho_{B f} \frac{\partial}{\partial \xi} \delta \rho_{B f}\right]=\Gamma \delta \rho_{B f}
$$

Para isso, fazemos a mudança de variável [117-124]:

$$
\xi=\Omega+\beta \int_{-\infty}^{\eta} \psi\left(\Omega, \eta^{\prime}\right) d \eta^{\prime}+\alpha \eta+\xi_{0}, t=\eta \text { e } \delta \rho_{B f}(\xi, t)=\psi(\Omega, \eta)
$$

com $\xi_{0}$ sendo uma constante arbitrária. Assim, temos os operadores:

$$
\frac{\partial}{\partial \xi}=\frac{1}{h(\Omega, \eta)} \frac{\partial}{\partial \Omega} \quad \text { e } \quad \frac{\partial}{\partial t}=\frac{\partial}{\partial \eta}-\frac{\beta}{h(\Omega, \eta)} \psi \frac{\partial}{\partial \Omega}-\frac{\alpha}{h(\Omega, \eta)} \frac{\partial}{\partial \Omega}
$$

onde a função $h(\Omega, \eta)$ é:

$$
h(\Omega, \eta)=1+\beta \int_{-\infty}^{\eta}\left[\frac{\partial}{\partial \Omega} \psi\left(\Omega, \eta^{\prime}\right)\right] d \eta^{\prime}
$$

Reescrevendo (D.1) com (D.3) e (D.4):

$$
h(\Omega, \eta)=\frac{1}{\Gamma \psi} \frac{\partial}{\partial \Omega} \frac{\partial \psi}{\partial \eta}
$$


De (D.4):

$$
\frac{\partial h}{\partial \eta}=\beta \frac{\partial \psi}{\partial \Omega}
$$

Então colocando (D.5) em (D.6):

$$
\psi \frac{\partial^{2}}{\partial \eta^{2}} \frac{\partial \psi}{\partial \Omega}-\frac{\partial \psi}{\partial \eta} \frac{\partial}{\partial \Omega} \frac{\partial \psi}{\partial \eta}-(\beta \Gamma)(\psi)^{2} \frac{\partial \psi}{\partial \Omega}=0
$$

que é a ROE reescrita em uma forma integrável. Utilizando o método da função da tangente hiperbólica [107-111], encontra-se as soluções exatas:

$$
\psi_{I}(\Omega, \eta)=-\frac{6 \gamma^{2} \lambda^{2}}{\beta \Gamma} \operatorname{sech}^{2}[\lambda(\Omega-\gamma \eta)] \quad \text { ou } \quad \psi_{I I}(\Omega, \eta)=\frac{4 \gamma^{2} \lambda^{2}}{\beta \Gamma}+\psi_{I}(\Omega, \eta)
$$

os parâmetros $\lambda$ (inverso do comprimento) e $\beta$ (velocidade) são constantes da integração com valores livres para se escolher. O termo negativo em $-\operatorname{sech}^{2}[\ldots]$ descreve um pulso de rarefação, cujo sinal negativo é devido a condição $(\alpha \Gamma)>0$.

Considenrando $\psi_{I}$ em (D.2), chegamos a uma solução paramétrica para a ROE (D.1):

$$
\delta \rho_{B f}(\xi, t)=-\frac{6 \gamma^{2} \lambda^{2}}{\beta \Gamma} \operatorname{sech}^{2}[\lambda(\Omega-\gamma t)]
$$

com

$$
\xi=\Omega+\alpha t+\xi_{0}+\frac{6 \gamma \lambda}{\beta \Gamma}\{\tanh [\lambda(\Omega-\gamma t)]-1\}
$$

$\psi_{I I}$ não foi considerada como solução, para evitar divergência devido ao termo constante em $\psi_{I I}$ na integral presente em (D.2):

$$
\beta \int_{-\infty}^{\eta} \frac{4 \gamma^{2} \lambda^{2}}{\beta \Gamma} d \eta^{\prime} \rightarrow \infty
$$




\section{Apêndice E}

\section{Equação de}

\section{Tolman-Oppenheimer-Volkoff (TOV)}

Para modelar a estrutura estelar com a relatividade geral é necessário que se assuma que a estrela seja esféricamente simétrica, isotrópica e composta de um fluido ideal. A equação TOV foi proposta por Oppenheimer e Volkoff [133], inspirados no trabalho realizado por Tolman [134]. A TOV juntamente com a equação de estado faz com que seja possível notar como a física microscópica influencia em propriedades macroscópicas, como a massa e raio do objeto.

Ao assumir que o sistema possui simetria esférica, a métrica do espaço-tempo pode ser formulada pela métrica de Schwarzschild $(\operatorname{com} c=G=1)$ :

$$
d s^{2}=e^{\nu(r)} d t^{2}-e^{\lambda(r)} d r^{2}-r^{2} d \theta^{2}-r^{2} \operatorname{sen}(\theta) d \phi^{2}
$$

onde $\nu$ e $\lambda$ são funções da métrica a serem determinadas.

Como também se assume que o sistema é composta de um fluido ideal pressurizado 
isotrópico, temos:

$$
T^{00}=\varepsilon(r)
$$

e

$$
T^{i j}=-p(r) \delta^{i j}
$$

sendo $\varepsilon$ e $p$, a densidade de energia e pressão.

Assim, precisamos resolver as equações de campo de Einstein:

$$
8 \pi T^{\mu \nu}=G^{\mu \nu}=R^{\mu \nu}-\frac{1}{2} R g^{\mu \nu}
$$

que utilizando a métrica (E.1), permite encontrar o tensor de Ricci $R^{\mu \nu}$ e os elementos de $g^{\mu \nu}$.

O primeiro elemento da equação é $[31,135]$ :

$$
8 \pi \varepsilon(r) e^{\nu(r)}=\frac{e^{\nu(r)}}{r^{2}}\left(1-\frac{d}{d r} r e^{-\lambda(r)}\right)
$$

que integrando:

$$
e^{-\lambda(r)}=1-\frac{2 M(r)}{r}
$$

Considerando o termo $\mu=\nu=1$ :

$$
-8 \pi p(r) e^{\lambda(r)}=\frac{-r \dot{\nu}(r)+e^{\lambda(r)}-1}{r^{2}}
$$

inserindo (E.6), chegamos:

$$
\frac{d \nu(r)}{d r}=\frac{1}{r}\left(\frac{2 M(r)}{r}+8 \pi r^{2} p(r)\right)\left(1-\frac{2 M(r)}{r}\right)^{-1}
$$

Utilizando que o sistema é isotrópico e estático, $\nabla_{\mu} T^{\mu \nu}=0$ :

$$
\nabla_{\mu} T^{\mu 1}=-\frac{d p(r)}{d r}-\frac{1}{2}(p+\varepsilon) \frac{d \nu(r)}{d r}=0
$$


Então, colocando (E.8) em (E.9), encontramos a equação TOV:

$$
\frac{d p(r)}{d r}=-\frac{1}{r^{2}}(p(r)+\varepsilon(r))\left(M(r)+4 \pi r^{3} p(r)\right)\left(1-\frac{2 M(r)}{r}\right)^{-1}
$$

sendo a massa dada pela equação da continuidade:

$$
\frac{d M(r)}{d r}=4 \pi r^{2} \varepsilon(r)
$$




\section{Apêndice F}

\section{Equações de Friedmann}

As equações de Friedmann relacionam a densidade de energia com o tempo. Para derivá-las, partimos da métrica geral:

$$
d s^{2}=g_{00} d t^{2}+2 g_{0 i} d x^{i} d t-g_{i j} d x^{i} d x^{j}
$$

onde $g_{00}=1$. Como, segundo o princípio cosmológico, o Universo é isotrópico e homogêneo [31], o termo $g_{0 i}=0$.

Considere uma esfera no espaço euclidiano em quatro dimensões nas coordenadas $(x, y, z, \omega) \rightarrow(\rho, \theta, \varphi, \omega)$, temos:

$$
d l^{2}=g_{i j} d x^{i} d x^{j}=d \rho^{2}+\rho^{2}\left[d \theta^{2}+\operatorname{sen}^{2}(\theta) d \varphi^{2}\right]+d \omega^{2}
$$

e também:

$$
x^{2}+y^{2}+z^{2}+\omega^{2}=\rho^{2}+\omega^{2}=a^{2} \rightarrow \rho d \rho+\omega d \omega=0 \rightarrow d \omega^{2}=\frac{\rho^{2} d \rho^{2}}{\omega^{2}}=\frac{\rho^{2} d \rho^{2}}{a^{2}-\rho^{2}}
$$

então:

$$
d l^{2}=d \rho^{2}+\rho^{2}\left[d \theta^{2}+\operatorname{sen}^{2}(\theta) d \varphi^{2}\right]+\frac{\rho^{2} d \rho^{2}}{a^{2}-\rho^{2}}
$$


ou

$$
d l^{2}=\frac{d \rho^{2}}{1-(\rho / a)^{2}}+\rho^{2}\left[d \theta^{2}+\operatorname{sen}^{2}(\theta) d \varphi^{2}\right]
$$

A expressão (F.5) representa um espaço homogêneo e isotrópico de curvatura $1 / a^{2}$ positiva. Caso seja necessário englobar a curvatura negativa ou nula faz a substituição $\rho=a r$. Em geral pode-se fazer $a \equiv a(t)$, que não pode depender das posições devido a homogeneidade, assim chega-se na métrica de Friedmann-Lemaître-Robertson-Walker (FLRW):

$$
d s^{2}=d t^{2}-a^{2}(t)\left\{\frac{d r^{2}}{1-k r^{2}}+r^{2}\left[d \theta^{2}+\operatorname{sen}^{2}(\theta) d \varphi^{2}\right]\right\}
$$

onde $k=-1,0,+1$ e $a(t)$ é o fator de escala.

Utilizando a conservação do tensor energia-momento:

$$
\nabla_{\nu} T^{\mu \nu}=T_{; \nu}^{\mu \nu}=T_{, \nu}^{\mu \nu}+T^{\sigma \nu} \Gamma_{\sigma \nu}^{\mu}+T^{\mu \sigma} \Gamma_{\sigma \nu}^{\nu}=0
$$

com a equação de Einstein [31]:

$$
R_{\mu \nu}-\frac{1}{2} g_{\mu \nu} R-g_{\mu \nu} \Lambda=8 \pi G T_{\mu \nu}
$$

e com a equação de estado:

$$
p \equiv p(\varepsilon)
$$

podemos descrever a expansão do Universo logo após o Big Bang. Onde, $\Gamma_{\sigma \nu}^{\mu}$ são os símbolos de Christoffel (que fazem o papel do campo de força gravitacional), $R_{\mu \nu}$ o tensor de curvatura de Ricci, $g_{\mu \nu}$ a métrica, $R$ a curvatura escalar, $\Lambda$ a constante cosmológica, $G$ a constante gravitacional de Newton, $p$ a pressão e $\varepsilon$ a densidade de energia.

Os símbolos de Christoffel são dados por [31]:

$$
\Gamma_{\alpha \beta}^{\mu}=\frac{1}{2} g^{\mu \nu}\left(\frac{\partial g_{\alpha \nu}}{\partial x^{\beta}}+\frac{\partial g_{\beta \nu}}{\partial x^{\alpha}}-\frac{\partial g_{\alpha \beta}}{\partial x^{\nu}}\right)
$$


$\operatorname{com} \alpha, \beta, \nu=0,1,2,3$.

Utilizando a métrica para este espaço, notamos que vários símbolos de Christoffel são nulos, conforme a Tabela F.1.

Tabela F.1: Símbolos de Christoffel nulos para métrica FLRW

\begin{tabular}{c||c||c||c}
\hline \hline$\mu=0$ & $\mu=1$ & $\mu=2$ & $\mu=3$ \\
\hline \hline$\Gamma_{00}^{0}$ & $\Gamma_{00}^{1}$ & $\Gamma_{00}^{2}$ & $\Gamma_{00}^{3}$ \\
\hline$\Gamma_{10}^{0}$ & $\Gamma_{02}^{1}$ & $\Gamma_{01}^{2}$ & $\Gamma_{01}^{3}$ \\
\hline$\Gamma_{20}^{0}$ & $\Gamma_{03}^{1}$ & $\Gamma_{03}^{2}$ & $\Gamma_{02}^{3}$ \\
\hline$\Gamma_{30}^{0}$ & $\Gamma_{12}^{1}$ & $\Gamma_{10}^{2}$ & $\Gamma_{10}^{3}$ \\
\hline$\Gamma_{01}^{0}$ & $\Gamma_{13}^{1}$ & $\Gamma_{11}^{2}$ & $\Gamma_{11}^{3}$ \\
\hline$\Gamma_{02}^{0}$ & $\Gamma_{20}^{1}$ & $\Gamma_{13}^{2}$ & $\Gamma_{12}^{3}$ \\
\hline$\Gamma_{03}^{0}$ & $\Gamma_{21}^{1}$ & $\Gamma_{22}^{2}$ & $\Gamma_{20}^{3}$ \\
\hline$\Gamma_{12}^{0}$ & $\Gamma_{23}^{1}$ & $\Gamma_{23}^{2}$ & $\Gamma_{21}^{3}$ \\
\hline$\Gamma_{13}^{0}$ & $\Gamma_{30}^{1}$ & $\Gamma_{30}^{2}$ & $\Gamma_{22}^{3}$ \\
\hline$\Gamma_{21}^{0}$ & $\Gamma_{31}^{1}$ & $\Gamma_{31}^{2}$ & $\Gamma_{33}^{3}$ \\
\hline$\Gamma_{23}^{0}$ & $\Gamma_{32}^{1}$ & $\Gamma_{32}^{2}$ & - \\
\hline$\Gamma_{31}^{0}$ & - & - & - \\
\hline$\Gamma_{32}^{0}$ & - & - & - \\
\hline \hline
\end{tabular}

Os símbolos não nulos são dados por:

$$
\begin{aligned}
& \Gamma_{11}^{0}=\frac{\dot{a}(t) a(t)}{1-k r^{2}}, \quad \Gamma_{22}^{0}=\dot{a}(t) a(t) r^{2}, \quad \Gamma_{33}^{0}=\dot{a}(t) a(t) r^{2} \sin ^{2}(\theta) \\
& \Gamma_{01}^{1}=\Gamma_{10}^{1}=\frac{\dot{a}(t)}{a(t)}, \quad \Gamma_{11}^{1}=\frac{k r}{1-k r^{2}}, \quad \Gamma_{22}^{1}=-r\left(1-k r^{2}\right), \\
& \Gamma_{33}^{1}=-r \sin ^{2}(\theta)\left(1-k r^{2}\right) \\
& \Gamma_{02}^{2}=\Gamma_{20}^{2}=\frac{\dot{a}(t)}{a(t)}, \quad \Gamma_{12}^{2}=\Gamma_{21}^{2}=\frac{1}{r}, \quad \Gamma_{33}^{2}=-\sin (\theta) \cos (\theta) \\
& \Gamma_{03}^{3}=\Gamma_{30}^{3}=\frac{\dot{a}(t)}{a(t)}, \quad \Gamma_{13}^{3}=\Gamma_{31}^{3}=\frac{1}{r}, \quad \Gamma_{23}^{3}=\Gamma_{32}^{3}=\cot (\theta)
\end{aligned}
$$


Então, o tensor de Ricci é escrito:

$$
R_{\nu \sigma}=\Gamma_{\nu \sigma ; \mu}^{\mu}-\Gamma_{\nu \mu ; \sigma}^{\mu}+\Gamma_{\nu \sigma}^{\alpha} \Gamma_{\alpha \mu}^{\mu}-\Gamma_{\nu \mu}^{\alpha} \Gamma_{\alpha \sigma}^{\mu}
$$

que utilizando a Tabela F.1 e as expressões (F.11), são nulos as seguintes componentes de (F.12):

$$
\begin{aligned}
& R_{01}=R_{02}=R_{03}=R_{10}=R_{12}=R_{13}=0 \\
& R_{20}=R_{21}=R_{23}=R_{30}=R_{31}=R_{32}=0
\end{aligned}
$$

ou seja, somente os tensores com índices iguais $(\nu=\sigma)$ são diferentes de zero.

Assim:

$$
\begin{aligned}
R_{00} & =-\Gamma_{01 ; 0}^{1}-\Gamma_{02 ; 0}^{2}-\Gamma_{03 ; 0}^{3}-\Gamma_{01}^{1} \Gamma_{10}^{1}-\Gamma_{02}^{2} \Gamma_{20}^{2}-\Gamma_{03}^{3} \Gamma_{30}^{3}=-3 \frac{\ddot{a}(t)}{a(t)} \\
R_{11} & =\Gamma_{11 ; 0}^{0}-\Gamma_{12 ; 1}^{2}-\Gamma_{13 ; 1}^{3}+\Gamma_{11}^{0} \Gamma_{02}^{2}+\Gamma_{11}^{0} \Gamma_{03}^{3}+\Gamma_{11}^{1} \Gamma_{12}^{2}+\Gamma_{11}^{1} \Gamma_{13}^{3}-\Gamma_{10}^{1} \Gamma_{11}^{0} \\
& -\Gamma_{12}^{2} \Gamma_{21}^{2}-\Gamma_{13}^{3} \Gamma_{31}^{3}=\frac{\ddot{a}(t) a(t)+2 \dot{a}^{2}(t)+2 k}{1-k r^{2}} \\
R_{22} & =\Gamma_{22 ; 0}^{0}+\Gamma_{22 ; 1}^{1}-\Gamma_{23 ; 2}^{3}+\Gamma_{22}^{0} \Gamma_{01}^{1}+\Gamma_{22}^{0} \Gamma_{03}^{3}+\Gamma_{22}^{1} \Gamma_{11}^{1}+\Gamma_{22}^{1} \Gamma_{12}^{2}+\Gamma_{22}^{1} \Gamma_{13}^{3} \\
& -\Gamma_{22}^{1} \Gamma_{12}^{2}-\Gamma_{20}^{2} \Gamma_{22}^{0}-\Gamma_{21}^{2} \Gamma_{22}^{1}-\Gamma_{23}^{3} \Gamma_{32}^{3}=\left[\ddot{a}(t) a(t)+2 \dot{a}^{2}(t)+2 k\right] r^{2} \\
R_{33} & =\Gamma_{33 ; 0}^{0}+\Gamma_{33 ; 1}^{1}+\Gamma_{33 ; 2}^{2}+\Gamma_{33}^{0} \Gamma_{01}^{1}+\Gamma_{33}^{0} \Gamma_{02}^{2}+\Gamma_{33}^{0} \Gamma_{03}^{3}+\Gamma_{33}^{1} \Gamma_{11}^{1}+\Gamma_{33}^{1} \Gamma_{12}^{2} \\
& +\Gamma_{33}^{1} \Gamma_{13}^{3}+\Gamma_{33}^{2} \Gamma_{23}^{3}-\Gamma_{33}^{0} \Gamma_{30}^{3}-\Gamma_{33}^{1} \Gamma_{31}^{3}-\Gamma_{33}^{2} \Gamma_{32}^{3}-\Gamma_{30}^{3} \Gamma_{33}^{0}-\Gamma_{31}^{3} \Gamma_{33}^{1}-\Gamma_{32}^{3} \Gamma_{33}^{2} \\
& =\left[\ddot{a}(t) a(t)+2 \dot{a}^{2}(t)+2 k\right] r^{2} \sin ^{2}(\theta)
\end{aligned}
$$

Utilizando (F.14) chegamos na curvatura escalar:

$$
R=g^{\mu \nu} R_{\mu \nu}=-\frac{6}{a^{2}(t)}\left[\ddot{a}(t) a(t)+\dot{a}^{2}(t)+k\right]
$$


Considerando a parte temporal de (F.8) e substituindo (F.14a) e (F.15), obtemos:

$$
\dot{a}^{2}(t)-\frac{8 \pi G \varepsilon}{3} a^{2}(t)+k-\frac{\Lambda}{3} a^{2}(t)=0
$$




\section{Apêndice G}

\section{Equação de Rayleigh-Plesset \\ Relativística (RRP) e a \\ Transformação de Sundman}

\section{G.1 Derivação da equação RRP}

A lagrangeana para um sistema simetricamente esférico pode ser obtida pelo princípio variacional e é dada por [160]:

$$
\mathcal{L}=-4 \pi \int_{0}^{\infty} d r r^{2} \varepsilon(n)
$$

onde $\varepsilon$ é a densidade de energia, $n$ é a densidade dada por $n=\rho / \gamma$, $\rho$ é o número de partículas por volume (densidade de número) e $\gamma$ é o fator de Lorentz $\gamma=\left(1-v^{2}\right)^{-1 / 2}$.

O sistema considerado é uma bolha relativística de raio $R$ com uma temperatura fixa, imersa em um meio com outra temperatura fixa. Então, a lagrangeana (G.1) 
pode ser decomposta nas contribuições das fases interna (in) e externa (ex):

$$
\mathcal{L}=\mathcal{L}_{\text {in }}+\mathcal{L}_{\text {ex }}=-4 \pi \int_{0}^{R} d r r^{2} \varepsilon_{\text {in }}\left(n_{\text {in }}\right)-\lim _{R_{\infty} \rightarrow \infty}\left\{4 \pi \int_{R}^{R_{\infty}} d r r^{2} \varepsilon_{\text {ex }}\left(n_{\text {ex }}\right)\right\}
$$

Por simplicidade será omitido o limite de $R_{\infty}$, retomando-o no final dos cálculos. A densidade de número para cada fase é:

$$
\rho_{i n}=\frac{N_{\text {in }}}{\left(\frac{4}{3} \pi R^{3}\right)} \quad \text { e } \quad \rho_{e x}=\frac{N_{e x}}{\left[\frac{4}{3} \pi\left(R_{\infty}^{3}-R^{3}\right)\right]}
$$

onde $N_{\text {in }}$ é o número de partículas dentro da bolha e $N_{e x}$ o número de partículas fora da bolha. O perfil da velocidade para cada fase é dado por (4.23) e (4.24), com os fatores de Lorentz (4.25).

A versão relativística da RRP é obtida pela equação de Euler-Lagrange, derivada da lagrangeana (G.2):

$$
\frac{d}{d t}\left(\frac{\partial \mathcal{L}}{\partial \dot{R}}\right)=\frac{\partial \mathcal{L}}{\partial R}
$$

Inserindo (G.2) em (G.4), temos:

$$
\frac{d}{d t}\left[\int_{0}^{R} d r r^{2} \frac{\partial \varepsilon_{i n}}{\partial \dot{R}}+\int_{R}^{R_{\infty}} d r r^{2} \frac{\partial \varepsilon_{e x}}{\partial \dot{R}}\right]=\frac{\partial}{\partial R}\left[\int_{0}^{R} d r r^{2} \varepsilon_{i n}+\int_{R}^{R_{\infty}} d r r^{2} \varepsilon_{e x}\right]
$$

Utilizando a regra de integração de Leibniz, calcula-se as duas derivadas com respeito a $R$ em (G.5):

$$
\begin{gathered}
\frac{\partial}{\partial R} \int_{0}^{R} d r r^{2} \varepsilon_{i n}=\int_{0}^{R} d r \frac{\partial}{\partial R}\left(r^{2} \varepsilon_{i n}\right)+R^{2}\left(\left.\varepsilon_{i n}\right|_{r=R}\right) \frac{\partial R}{\partial R}-0^{2}\left(\left.\varepsilon_{i n}\right|_{r=0}\right) \frac{\partial 0}{\partial R} \\
\frac{\partial}{\partial R} \int_{0}^{R} d r r^{2} \varepsilon_{i n}=\int_{0}^{R} d r r^{2} \frac{\partial \varepsilon_{i n}}{\partial R}+R^{2}\left(\left.\varepsilon_{i n}\right|_{r=R}\right)
\end{gathered}
$$

e também:

$$
\frac{\partial}{\partial R} \int_{R}^{R_{\infty}} d r r^{2} \varepsilon_{e x}=\int_{R}^{R_{\infty}} d r \frac{\partial}{\partial R}\left(r^{2} \varepsilon_{e x}\right)+R_{\infty}^{2}\left(\left.\varepsilon_{e x}\right|_{r=R_{\infty}}\right) \frac{\partial R_{\infty}}{\partial R}-R^{2}\left(\left.\varepsilon_{e x}\right|_{r=R}\right) \frac{\partial R}{\partial R}
$$




$$
\frac{\partial}{\partial R} \int_{R}^{R_{\infty}} d r r^{2} \varepsilon_{e x}=\int_{R}^{R_{\infty}} d r r^{2} \frac{\partial \varepsilon_{e x}}{\partial R}-R^{2}\left(\left.\varepsilon_{e x}\right|_{r=R}\right)
$$

Mudando a notação de $\varepsilon_{i n}=\varepsilon_{i n}\left(n_{i n}\right), \varepsilon_{e x}=\varepsilon_{e x}\left(n_{e x}\right)$ e inserindo (G.6) e (G.7) em (G.5), obtemos:

$$
\begin{gathered}
\frac{d}{d t} \int_{0}^{R} d r r^{2} \frac{\partial \varepsilon_{i n}}{\partial n_{i n}} \frac{\partial n_{i n}}{\partial \dot{R}}+\frac{d}{d t} \int_{R}^{R_{\infty}} d r r^{2} \frac{\partial \varepsilon_{e x}}{\partial n_{e x}} \frac{\partial n_{e x}}{\partial \dot{R}}=R^{2}\left(\left.\varepsilon_{i n}\right|_{r=R}-\left.\varepsilon_{e x}\right|_{r=R}\right) \\
+\int_{0}^{R} d r r^{2} \frac{\partial \varepsilon_{i n}}{\partial n_{i n}} \frac{\partial n_{i n}}{\partial R}+\int_{R}^{R_{\infty}} d r r^{2} \frac{\partial \varepsilon_{e x}}{\partial n_{e x}} \frac{\partial n_{e x}}{\partial R}
\end{gathered}
$$

Para continuar com os cálculos em temperatura finita, usa-se as relações termodinâmicas [161]:

$$
d \varepsilon=T d s+\mu d n, \quad \text { onde } \quad \mu=\frac{\partial \varepsilon}{\partial n}
$$

e a relação de Gibbs $(\varepsilon+p=\mu n+T s)$ fica:

$$
\frac{\partial \varepsilon}{\partial n}=\frac{(\varepsilon+p)}{n}-\frac{T s}{n}
$$

A densidade de entropia é dada por [161]:

$$
s=\frac{\partial p}{\partial T}
$$

e inserindo (G.10) em (G.9):

$$
\frac{\partial \varepsilon}{\partial n}=\frac{(\varepsilon+p)}{n}-\frac{T}{n} \frac{\partial p}{\partial T}
$$

essa relação introduzirá a temperatura nos cálculos. Fazendo a substituição de (G.11) em (G.8), chegamos:

$$
\frac{d}{d t} \int_{0}^{R} d r r^{2} \frac{\left(\varepsilon_{i n}+p_{i n}\right)}{n_{i n}} \frac{\partial n_{i n}}{\partial \dot{R}}-\frac{d}{d t} \int_{0}^{R} d r r^{2} \frac{T}{n_{i n}} \frac{\partial p_{i n}}{\partial T} \frac{\partial n_{i n}}{\partial \dot{R}}+\frac{d}{d t} \int_{R}^{R_{\infty}} d r r^{2} \frac{\left(\varepsilon_{e x}+p_{e x}\right)}{n_{e x}} \frac{\partial n_{e x}}{\partial \dot{R}}
$$




$$
\begin{aligned}
& -\frac{d}{d t} \int_{R}^{R_{\infty}} d r r^{2} \frac{T}{n_{e x}} \frac{\partial p_{e x}}{\partial T} \frac{\partial n_{e x}}{\partial \dot{R}}=R^{2}\left(\left.\varepsilon_{i n}\right|_{r=R}-\left.\varepsilon_{e x}\right|_{r=R}\right)+\int_{0}^{R} d r r^{2} \frac{\left(\varepsilon_{i n}+p_{i n}\right)}{n_{i n}} \frac{\partial n_{i n}}{\partial R} \\
& -\int_{0}^{R} d r r^{2} \frac{T}{n_{\text {in }}} \frac{\partial p_{i n}}{\partial T} \frac{\partial n_{i n}}{\partial R}+\int_{R}^{R_{\infty}} d r r^{2} \frac{\left(\varepsilon_{e x}+p_{e x}\right)}{n_{e x}} \frac{\partial n_{e x}}{\partial R}-\int_{R}^{R_{\infty}} d r r^{2} \frac{T}{n_{e x}} \frac{\partial p_{e x}}{\partial T} \frac{\partial n_{e x}}{\partial R}
\end{aligned}
$$

De [160] tem-se os resultados:

$$
\begin{gathered}
\frac{\partial n_{i n}}{\partial R}=-\frac{3}{R} n_{i n}+\left(\gamma_{i n} \frac{\dot{R}}{R}\right)^{2} \frac{r^{2}}{R} n_{i n} \\
\frac{\partial n_{i n}}{\partial \dot{R}}=-\left(\gamma_{i n} \frac{r}{R}\right)^{2} \dot{R} n_{i n} \\
\frac{\partial n_{e x}}{\partial R}=\frac{3 R^{2}}{\left(R_{\infty}^{3}-R^{3}\right)} n_{e x}-\frac{R^{3}\left(2 R_{\infty}^{3}+R^{3}\right)\left(R_{\infty}^{3}-R^{3}\right)^{2}}{r^{4}\left(R_{\infty}^{3}-R^{3}\right)^{3}}\left(\gamma_{e x} \dot{R}\right)^{2} n_{e x}
\end{gathered}
$$

$\mathrm{e}$

$$
\frac{\partial n_{e x}}{\partial \dot{R}}=-\left(\frac{\gamma_{e x} R^{2}}{r^{2}}\right)^{2}\left[\frac{\left(R_{\infty}^{3}-r^{3}\right)}{\left(R_{\infty}^{3}-R^{3}\right)}\right]^{2} \dot{R} n_{e x}
$$

Inserindo (G.13) em (G.16) e (G.12):

$$
\begin{gathered}
-\frac{d}{d t}\left\{\frac{\dot{R}}{R^{2}} \int_{0}^{R} d r r^{4}\left(\varepsilon_{i n}+p_{i n}-T \frac{\partial p_{i n}}{\partial T}\right) \gamma_{i n}{ }^{2}\right. \\
\left.+R^{4} \dot{R} \int_{R}^{R_{\infty}} \frac{d r}{r^{2}}\left(\varepsilon_{e x}+p_{e x}-T \frac{\partial p_{e x}}{\partial T}\right) \gamma_{e x}^{2}\left[\frac{\left(R_{\infty}^{3}-r^{3}\right)}{\left(R_{\infty}^{3}-R^{3}\right)}\right]^{2}\right\}= \\
=R^{2}\left(\left.\varepsilon_{i n}\right|_{r=R}-\left.\varepsilon_{e x}\right|_{r=R}\right)+\int_{0}^{R} d r r^{2}\left(\varepsilon_{i n}+p_{i n}-T \frac{\partial p_{i n}}{\partial T}\right)\left[-\frac{3}{R}+\left(\gamma_{i n} \frac{\dot{R}}{R}\right)^{2} \frac{r^{2}}{R}\right] \\
+\int_{R}^{R_{\infty}} d r r^{2}\left(\varepsilon_{e x}+p_{e x}-T \frac{\partial p_{e x}}{\partial T}\right)\left[\frac{3 R^{2}}{\left(R_{\infty}^{3}-R^{3}\right)}-\frac{R^{3}\left(2 R_{\infty}^{3}+R^{3}\right)\left(R_{\infty}^{3}-R^{3}\right)^{2}}{r^{4}\left(R_{\infty}^{3}-R^{3}\right)^{3}}\left(\gamma_{e x} \dot{R}\right)^{2}\right]
\end{gathered}
$$

Considerando a aproximação para grande $R_{\infty}$ antes de fazer o limite $R_{\infty} \rightarrow \infty$ :

$$
\left[\frac{\left(R_{\infty}^{3}-r^{3}\right)}{\left(R_{\infty}^{3}-R^{3}\right)}\right]^{2} \cong \frac{\left(R_{\infty}^{3}\right)^{2}}{\left(R_{\infty}^{3}\right)^{2}} \cong 1
$$




$$
\frac{3 R^{2}}{\left(R_{\infty}^{3}-R^{3}\right)} \cong \frac{3 R^{2}}{R_{\infty}^{3}}
$$

e

$$
-\frac{R^{3}\left(2 R_{\infty}^{3}+R^{3}\right)\left(R_{\infty}^{3}-R^{3}\right)^{2}}{r^{4}\left(R_{\infty}^{3}-R^{3}\right)^{3}} \cong-\frac{R^{3}\left(2 R_{\infty}^{3}\right)\left(R_{\infty}^{3}\right)^{2}}{r^{4}\left(R_{\infty}^{3}\right)^{3}} \cong-\frac{2 R^{3}}{r^{4}} \frac{\left(R_{\infty}^{3}\right)^{3}}{\left(R_{\infty}^{3}\right)^{3}} \cong-\frac{2 R^{3}}{r^{4}}
$$

De (4.25) para grande $R_{\infty}$, o novo fator de Lorentz para a fase de líquido (externa) se torna:

$\gamma_{e x}=\left[1-\left(\frac{R^{2}\left(R_{\infty}^{3}-r^{3}\right)}{r^{2}\left(R_{\infty}^{3}-R^{3}\right) \dot{R}}\right)^{2}\right]^{-1 / 2} \cong\left[1-\left(\frac{R^{2} R_{\infty}^{3}}{r^{2} R_{\infty}^{3} \dot{R}}\right)^{2}\right]^{-1 / 2}=\left[1-\left(\frac{R^{2}}{r^{2} \dot{R}}\right)^{2}\right]^{-1 / 2}$

Reescrevendo (G.17) usando (G.18) em (G.21), chegamos em:

$$
\begin{gathered}
-\frac{d}{d t}\left\{\frac{\dot{R}}{R^{2}} \int_{0}^{R} d r r^{4}\left(\varepsilon_{i n}+p_{i n}-T \frac{\partial p_{G}}{\partial T}\right) \gamma_{i n}{ }^{2}+R^{4} \dot{R} \int_{R}^{R_{\infty}} \frac{d r}{r^{2}}\left(\varepsilon_{e x}+p_{e x}-T \frac{\partial p_{e x}}{\partial T}\right) \gamma_{e x}{ }^{2}\right\}= \\
=R^{2}\left(\left.\varepsilon_{i n}\right|_{r=R}-\left.\varepsilon_{e x}\right|_{r=R}\right)+\int_{0}^{R} d r r^{2}\left(\varepsilon_{i n}+p_{i n}-T \frac{\partial p_{i n}}{\partial T}\right)\left[-\frac{3}{R}+\left(\gamma_{i n} \frac{\dot{R}}{R}\right)^{2} \frac{r^{2}}{R}\right] \\
+\int_{R}^{R_{\infty}} d r r^{2}\left(\varepsilon_{e x}+p_{L}-T \frac{\partial p_{e x}}{\partial T}\right)\left[\frac{3 R^{2}}{R_{\infty}^{3}}-\frac{2 R^{3}}{r^{4}}\left(\gamma_{e x} \dot{R}\right)^{2}\right]
\end{gathered}
$$

Seguindo o feito em [160] faz-se a proximação:

$$
\begin{gathered}
\left.\int_{R}^{R_{\infty}} d r r^{2}\left(\varepsilon_{e x}+p_{e x}-T \frac{\partial p_{e x}}{\partial T}\right) \frac{3 R^{2}}{R_{\infty}^{3}} \cong \frac{3 R^{2}}{R_{\infty}^{3}}\left(\varepsilon_{e x}+p_{e x}-T \frac{\partial p_{e x}}{\partial T}\right)\right|_{R_{\infty}} \int_{R}^{R_{\infty}} d r r^{2} \\
=\left.\frac{3 R^{2}}{R_{\infty}^{3}}\left(\varepsilon_{e x}+p_{e x}-T \frac{\partial p_{e x}}{\partial T}\right)\right|_{R_{\infty}}\left(\frac{R_{\infty}^{3}}{3}-\frac{R^{3}}{3}\right)= \\
=\left.R^{2}\left(\varepsilon_{e x}+p_{e x}-T \frac{\partial p_{e x}}{\partial T}\right)\right|_{R_{\infty}}-\left.\frac{R^{5}}{R_{\infty}^{3}}\left(\varepsilon_{e x}+p_{e x}-T \frac{\partial p_{e x}}{\partial T}\right)\right|_{R_{\infty}}
\end{gathered}
$$

Com (G.23) em (G.22) e tomando o limite $R_{\infty} \rightarrow \infty$ na expressão resultada, 
obtemos:

$$
\begin{aligned}
- & \frac{d}{d t}\left\{\frac{\dot{R}}{R^{2}} \int_{0}^{R} d r r^{4}\left(\varepsilon_{i n}+p_{i n}-T \frac{\partial p_{i n}}{\partial T}\right) \gamma_{i n}^{2}+R^{4} \dot{R} \int_{R}^{\infty} \frac{d r}{r^{2}}\left(\varepsilon_{e x}+p_{e x}-T \frac{\partial p_{e x}}{\partial T}\right) \gamma_{e x}{ }^{2}\right\}= \\
= & \left.R^{2}\left(\varepsilon_{i n}-\varepsilon_{e x}\right)\right|_{R}-\frac{3}{R} \int_{0}^{R} d r r^{2}\left(\varepsilon_{i n}+p_{i n}-T \frac{\partial p_{i n}}{\partial T}\right)+\frac{\dot{R}^{2}}{R^{3}} \int_{0}^{R} d r r^{4}\left(\varepsilon_{i n}+p_{i n}-T \frac{\partial p_{i n}}{\partial T}\right) \gamma_{i n}{ }^{2} \\
& +\left.R^{2}\left(\varepsilon_{e x}+p_{e x}-T \frac{\partial p_{e x}}{\partial T}\right)\right|_{\infty}-2 R^{3} \dot{R}^{2} \int_{R}^{\infty} \frac{d r}{r^{2}}\left(\varepsilon_{e x}+p_{e x}-T \frac{\partial p_{e x}}{\partial T}\right) \gamma_{e x}{ }^{2}
\end{aligned}
$$

Utilizando a nova variável $x=r / R$ é possível reescrever as integrais que contêm os fatores de Lorentz e então (G.24) se torna:

$$
\begin{aligned}
& -\frac{d}{d t}\left\{R^{3} \dot{R}\left[\int_{0}^{1} d x x^{4}\left(\varepsilon_{i n}+p_{i n}-T \frac{\partial p_{i n}}{\partial T}\right) \gamma_{i n}{ }^{2}+\int_{1}^{\infty} \frac{d x}{x^{2}}\left(\varepsilon_{e x}+p_{e x}-T \frac{\partial p_{e x}}{\partial T}\right) \gamma_{e x}{ }^{2}\right]\right\}= \\
& =R^{2}\left[\left.\left(\varepsilon_{i n}-\varepsilon_{e x}\right)\right|_{R}+\left.\left(\varepsilon_{e x}+p_{e x}-T \frac{\partial p_{e x}}{\partial T}\right)\right|_{\infty}\right]-\frac{3}{R} \int_{0}^{R} d r r^{2}\left(\varepsilon_{i n}+p_{i n}-T \frac{\partial p_{i n}}{\partial T}\right) \\
& +R^{2} \dot{R}^{2}\left[\int_{0}^{1} d x x^{4}\left(\varepsilon_{i n}+p_{i n}-T \frac{\partial p_{i n}}{\partial T}\right) \gamma_{i n}{ }^{2}-2 \int_{1}^{\infty} \frac{d x}{x^{2}}\left(\varepsilon_{e x}+p_{e x}-T \frac{\partial p_{e x}}{\partial T}\right) \gamma_{e x}{ }^{2}\right]
\end{aligned}
$$

onde podemos reescrever as integrais como:

$$
\begin{aligned}
& I_{1} \equiv \int_{0}^{1} d x x^{4}\left(\varepsilon_{i n}+p_{i n}{ }^{e f}\right) \gamma_{i n}{ }^{2} \\
& I_{2} \equiv \int_{1}^{\infty} \frac{d x}{x^{2}}\left(\varepsilon_{e x}+p_{e x}{ }^{e f}\right) \gamma_{e x}{ }^{2}
\end{aligned}
$$

sendo a "pressão efetiva" dada por:

$$
p_{e x}{ }^{e f} \equiv p_{e x}-\left.T_{e x} \frac{\partial p_{L}}{\partial T}\right|_{T=T_{e x}} \quad \text { e } \quad p_{i n}{ }^{e f} \equiv p_{i n}-\left.T_{i n} \frac{\partial p_{G}}{\partial T}\right|_{T=T_{i n}}
$$


para simplificar a notação, também podemos escrever:

$$
F \equiv-R^{2}\left[\left.\left(\varepsilon_{i n}-\varepsilon_{e x}\right)\right|_{R}+\left.\left(\varepsilon_{e x}+p_{e x}-T \frac{\partial p_{e x}}{\partial T}\right)\right|_{\infty}\right]+\frac{3}{R} \int_{0}^{R} d r r^{2}\left(\varepsilon_{i n}+p_{i n}-T \frac{\partial p_{i n}}{\partial T}\right)
$$

$\mathrm{Ou}$

$$
F=\left[\left(p_{i n}-p_{e x}\right)-\left(\left.T_{i n} \frac{\partial p_{i n}}{\partial T}\right|_{T=T_{i n}}-\left.T_{e x} \frac{\partial p_{e x}}{\partial T}\right|_{T=T_{e x}}\right)\right] R^{2}=-\left(p_{e x}^{e f}-p_{i n}^{e f}\right) R^{2}
$$

assim encontramos a equação RRP:

$$
\frac{d}{d t}\left[\left(I_{1}+I_{2}\right) R^{3} \dot{R}\right]+\left(I_{1}-2 I_{2}\right) R^{2} \dot{R}^{2}=F
$$

\section{G.2 Transformação de Sundman}

A equação para o limite não relativístico da RRP na forma compacta é:

$$
R \ddot{R}+\alpha \dot{R}^{2}=\beta
$$

multiplicando por $\dot{R} R^{(2 \alpha-1)}$ :

$$
R^{2 \alpha} \dot{R} \ddot{R}+\alpha R^{(2 \alpha-1)} \dot{R}^{3}=\beta R^{(2 \alpha-1)} \dot{R}
$$

e integrando em $t$ :

$$
\dot{R}^{2}=2 \mathcal{C} R^{-2 \alpha}+\frac{\beta}{\alpha}
$$

onde $\mathcal{C}$ é uma contante de integração.

Utilizando a transformação de Sundman [162] e uma transformação do tipo de potência:

$$
d t=R^{\delta} d \tau \quad \text { e } \quad R=\nu^{\eta}
$$


derivando a segunda transformação e então a dividindo pela primeira transformação, obtemos:

$$
\frac{d R}{d t}=\frac{\eta \nu^{\eta-1} d \nu}{R^{\delta} d \tau}=\eta \nu^{\eta(1-\delta)-1} \frac{d \nu}{d \tau}
$$

assim (G.34) pode ser escrita como:

$$
\left(\frac{d \nu}{d \tau}\right)^{2}=\frac{2 \mathcal{C}}{\eta^{2}} \nu^{-2 \eta \alpha-2 \eta+2 \eta \delta+2}+\left(\frac{\beta}{\alpha \eta^{2}}\right) \nu^{-2 \eta+2 \eta \delta+2}
$$

O método de Sundman, diz que os polinômios devem ser de ordem "3" e "4" [162], então:

$$
\begin{gathered}
-2 \eta \alpha-2 \eta+2 \eta \delta+2=3 \quad \text { e } \quad-2 \eta+2 \eta \delta+2=4 \\
\eta=\frac{1}{2 \alpha} \quad \text { e } \quad \delta=2 \alpha+1
\end{gathered}
$$

deste modo (G.37) pode ser manipulada chegando em:

$$
\pm \int_{\nu_{0}}^{\nu} \frac{d \nu}{\sqrt{\left(8 \mathcal{C} \alpha^{2}\right) \nu^{3}+(4 \alpha \beta) \nu^{4}}}=\int_{\tau_{0}}^{\tau} d \tau
$$

usando o resultado $\int \frac{d x}{\sqrt{a x^{3}+b x^{4}}}=\frac{-2 \sqrt{x^{3}(a+b x)}}{a x^{2}}$ e $t_{0}=0 \rightarrow R_{0}$ e $\tau_{0}=0$, temos:

$$
\nu=\frac{2 \mathcal{C} \alpha}{4 \mathcal{C}^{2} \alpha^{3}(\tau-\mathcal{A})^{2}-\beta}
$$

onde $\mathcal{A}=\frac{2 \sqrt{\nu_{0}^{3}\left[\left(8 \mathcal{C} \alpha^{2}\right)+(4 \alpha \beta) \nu_{0}\right]}}{\left(8 \mathcal{C} \alpha^{2}\right) \nu_{0}^{2}} \operatorname{com} \nu_{0}=R_{0}^{-\eta}=R_{0}^{-1 / 2 \alpha}$.

Como $\nu=R^{-\eta}=R^{-1 / 2 \alpha}$, (G.40) é reescrita na forma:

$$
R(\tau)=\left[\frac{2 \mathcal{C} \alpha}{4 \mathcal{C}^{2} \alpha^{3}(\tau-\mathcal{A})^{2}-\beta}\right]^{\frac{1}{2 \alpha}}
$$

Substituindo o segundo resultado de (G.38) no primeiro de (G.35):

$$
t=\int_{0}^{\tau} d \tau R^{2 \alpha+1}(\tau)
$$


portanto (G.41) é:

$$
t(\tau)=\int_{0}^{\tau} d \tau\left[\frac{2 \mathcal{C} \alpha}{4 \mathcal{C}^{2} \alpha^{3}(\tau-\mathcal{A})^{2}-\beta}\right]^{1+\frac{1}{2 \alpha}}
$$

Agora, fazendo a mudança de variável $(\tau-\mathcal{A}=y)$ em (G.43):

$$
t(\tau)=\int_{-\mathcal{A}}^{0} d y\left[\frac{2 \mathcal{C} \alpha}{4 \mathcal{C}^{2} \alpha^{3} y^{2}-\beta}\right]^{1+\frac{1}{2 \alpha}}+\int_{0}^{\tau-\mathcal{A}} d y\left[\frac{2 \mathcal{C} \alpha}{4 \mathcal{C}^{2} \alpha^{3} y^{2}-\beta}\right]^{1+\frac{1}{2 \alpha}}
$$

Na primeira integral de (G.44) fazemos a mudança de variável $\left(-y=\mathcal{A} X^{1 / 2}\right)$ :

$$
\int_{-\mathcal{A}}^{0} d y\left[\frac{2 \mathcal{C} \alpha}{4 \mathcal{C}^{2} \alpha^{3} y^{2}-\beta}\right]^{1+\frac{1}{2 \alpha}}=\frac{\mathcal{A}}{2}\left(\frac{-2 \mathcal{C} \alpha}{\beta}\right)^{1+\frac{1}{2 \alpha}} \int_{0}^{1} d X \frac{X^{-1 / 2}}{\left(1-\frac{4 \mathcal{C}^{2} \alpha^{3} \mathcal{A}^{2}}{\beta} X\right)^{1+\frac{1}{2 \alpha}}}
$$

e na segunda integral de (G.44) fazemos outra mudança de variável $y=(\tau-\mathcal{A}) \xi^{1 / 2}$ :

$$
\int_{0}^{\tau-\mathcal{A}} d y\left[\frac{2 \mathcal{C} \alpha}{4 \mathcal{C}^{2} \alpha^{3} y^{2}-\beta}\right]^{1+\frac{1}{2 \alpha}}=\frac{\tau-\mathcal{A}}{2}\left(\frac{-2 \mathcal{C} \alpha}{\beta}\right)^{1+\frac{1}{2 \alpha}} \int_{0}^{1} d \xi \frac{\xi^{-1 / 2}}{\left(1-\frac{4 \mathcal{C}^{2} \alpha^{3}(\tau-\mathcal{A})^{2}}{\beta} \xi\right)^{1+\frac{1}{2 \alpha}}}
$$

Lembrando da definição da função hipergeométrica (4.42), podemos relacionar de (G.45) e (G.46):

$$
\begin{aligned}
& b-1=-\frac{1}{2} \rightarrow b=\frac{1}{2} \\
& a=1+\frac{1}{2 \alpha} \\
& c-b-1=0 \rightarrow c=\frac{3}{2}
\end{aligned}
$$

e das funções $\Gamma$ de (4.42):

$$
\frac{\Gamma(b) \Gamma(c-b)}{\Gamma(c)}=\frac{\Gamma(1 / 2) \Gamma(1)}{\Gamma(3 / 2)}=\frac{\sqrt{\pi}}{\sqrt{\pi} / 2}=2
$$


A constante $\mathcal{C}$ pode ser calculada como segue:

$$
\begin{gathered}
\left(\frac{d R}{d t}\right)^{2}=2 \mathcal{C} R^{-2 \alpha}+\left.\frac{\beta}{\alpha} \longrightarrow \frac{d R}{d t}\right|_{t=t_{0}=0}=v_{0} \\
\mathcal{C}=\left(v_{0}^{2}-\frac{\beta}{\alpha}\right) \frac{R^{2 \alpha}}{2}
\end{gathered}
$$

onde $v_{0}$ é a taxa de variação inicial do raio.

Com (G.41), encotramos $(\tau-\mathcal{A})$ :

$$
(\tau-\mathcal{A})=\sqrt{\frac{1}{2 \mathcal{C} \alpha^{2} R^{2 \alpha}}+\frac{\beta}{4 \mathcal{C}^{2} \alpha^{3}}}
$$

Substituindo os resultados de (G.45) até (G.52) em (G.44), encontramos a expressão:

$$
\begin{gathered}
t(R)=\left(\frac{-2 \mathcal{C} \alpha}{\beta}\right)^{1+\frac{1}{2 \alpha}}\left\{\sqrt{\frac{1}{2 \mathcal{C} \alpha^{2} R^{2 \alpha}}+\frac{\beta}{4 \mathcal{C}^{2} \alpha^{3}}}{ }_{2} F_{1}\left(1+\frac{1}{2 \alpha}, \frac{1}{2} ; \frac{3}{2} ; \frac{4 \mathcal{C}^{2} \alpha^{3}}{\beta}\left[\frac{1}{2 \mathcal{C} \alpha^{2} R^{2 \alpha}}+\frac{\beta}{4 \mathcal{C}^{2} \alpha^{3}}\right]\right)\right. \\
\left.+\mathcal{A}_{2} F_{1}\left(1+\frac{1}{2 \alpha}, \frac{1}{2} ; \frac{3}{2} ; \frac{4 \mathcal{C}^{2} \alpha^{3} \mathcal{A}^{2}}{\beta}\right)\right\}
\end{gathered}
$$

que relaciona o tempo com o raio. 


\section{Bibliografia}

[1] M. E. Peskin e D. V. Schroeder, "An Introduction to Quantum Field Theory", Addison-Wesley (1995).

[2] M. Gell-Mann, Phys. Lett. 8, 214 (1964).

[3] S. Weinberg, Phys. Rev. Lett. 31, 494 (1973).

[4] T. W. B. Kibble, Phys. Rev. 155, 1554 (1967).

[5] D. J. Gross and F. Wilczek, Phys. Rev. Lett. 30, 1343 (1973).

[6] D. J. Gross and F. Wilczek, Phys. Rev. D 8, 3633 (1973).

[7] H. D. Politzer, Phys. Rev. Lett. 30, 1346 (1973).

[8] K. G. Wilson, Phys. Rev. D 10, 2445 (1974).

[9] T. Muta, "Foundations of Quantum Chromodynamics", World Scientific Publishing Co. Pte. Ltd. , 1987.

[10] F. Halzen and A. D. Martin, "Quarks And Leptons: An Introductory Course In Modern Particle Physics", New York, Usa: Wiley (1984) 396p.

[11] F. E. Close, "An Introduction to Quarks and Partons", Academic Press/london 1979, 481p.

[12] D. Griffiths, "Introduction to Elementary Particles", John Wiley \& Sons Inc. , 1987. 
[13] W. Greiner, S. Schramm and E. Stein, "Quantum Chromodynamics", Springer, 1989.

[14] G. Martinez, arXiv:1304.1452 [nucl-ex].

[15] E. V. Shuryak, Phys. Lett. 78B, 150 (1978) [Sov. J. Nucl. Phys. 28, 408 (1978)] [Yad. Fiz. 28, 796 (1978)].

[16] B. Muller, J. Schukraft and B. Wyslouch, Ann. Rev. Nucl. Part. Sci. 62, 361 (2012).

[17] M. Gyulassy and L. McLerran, Nucl. Phys. A 750, 30 (2005).

[18] J. Adams et al. [STAR Collaboration], Nucl. Phys. A 757, 102 (2005).

[19] K. Adcox et al. [PHENIX Collaboration], Nucl. Phys. A 757, 184 (2005).

[20] I. C. Arsene et al. [BRAHMS Collaboration], Phys. Lett. B 677, 267 (2009).

[21] B. B. Back et al., Nucl. Phys. A 757, 28 (2005).

[22] A. Grelli [ALICE Collaboration], arXiv:1711.03369 [hep-ex].

[23] S. I. Finazzo, "Estudando plasmas não-Abelianos fortemente acoplados usando dualidade gauge/gravity", 2014. Tese (Doutorado) - Instituto de Física, Universidade de São Paulo, São Paulo.

[24] D. Ivanenko e D. E. Kurdgelaidze, Astrophysics (Russ.), 1, (1965).

[25] D. Ivanenko e D. E. Kurdgelaidze, Nuevo Cimento, 2, (1969).

[26] N. Itoh, Prog. Theor. Phys. 44, 291 (1970).

[27] M. Alford, D. Blaschke, A. Drago, T. Klahn, G. Pagliara and J. Schaffner-Bielich, Nature 445, E7 (2007).

[28] H. Li, X. L. Luo, Y. Jiang and H. S. Zong, Phys. Rev. D 83, 025012 (2011). 
[29] F. Weber, Prog. Part. Nucl. Phys. 54, 193 (2005).

[30] W. Buchmüller, Acta Phys. Polon. B 43, 2153 (2012).

[31] S. Weinberg, "Gravitation and Cosmology: Principles and Applications of The General Theory of Relativity", New York: Wiley, 1972.

[32] S. Weinberg, "Cosmology", New York: Oxford, 2008.

[33] J. N. Islam, "An Introduction to Mathematical Cosmology", Cambridge, 2001.

[34] B. Franzon, D. A. Fogaça, F. S. Navarra and J. E. Horvath, Phys. Rev. D 86, 065031 (2012).

[35] H. B. Meyer, Eur. Phys. J. A 47, 86 (2011).

[36] I. Tews, J. Carlson, S. Gandolfi and S. Reddy, Astrophys. J. 860, no. 2, 149 (2018).

[37] G. P. Salam, PoS ICHEP 2010, 556 (2010).

[38] E. S. Fraga, A. Kurkela and A. Vuorinen, Eur. Phys. J. A 52, 49 (2016).

[39] B. P. Abbott et al. [LIGO Scientific and Virgo Collaborations], Phys. Rev. Lett. 119, no. 16, 161101 (2017).

[40] T. Hinderer, Astrophys. J. 677, 1216 (2008); T. Hinderer, B. D. Lackey, R. N. Lang, and J. S. Read, Phys. Rev. D 81, 123016 (2010); S. Postnikov, M. Prakash, and J. M. Lattimer, Phys. Rev. D 82, 024016 (2010).

[41] D. A. Fogaça and F. S. Navarra, Phys. Lett. B 700, 236 (2011).

[42] D. A. Fogaça, S. M. Sanches and F. S. Navarra, Nucl. Phys. A 973, 48 (2018).

[43] D. A. Fogaça, S. M. Sanches and F. S. Navarra, Commun. Nonlinear Sci. Numer. Simul. 66, 208 (2019). 
[44] D. A. Fogaça, S. M. Sanches, T. F. Motta and F. S. Navarra, Phys. Rev. C 94, 055805 (2016).

[45] S. M. Sanches, F. S. Navarra and D. A. Fogaça, Nucl. Phys. A 937, 1 (2015).

[46] D. A. Fogaça, S. M. Sanches, R. Fariello and F. S. Navarra, Phys. Rev. C 93, 055204 (2016).

[47] S. M. Sanches, D. A. Fogaça, F. S. Navarra and H. Marrochio, Phys. Rev. C 92, $025204(2015)$.

[48] L. S. Celenza and C. M. Shakin, Phys. Rev. D 34, 1591 (1986).

[49] X. d. Li and C. M. Shakin, Phys. Rev. D 71, 074007 (2005).

[50] D. E. Miller, Phys. Rept. 443, 55 (2007).

[51] B.D. Serot and J.D. Walecka, Advances in Nuclear Physics 16, 1 (1986).

[52] J.D. Walecka, "Theoretical Nuclear and Subnuclear Physics", Second Edition, Imperial College Press and World Scientific Publishing Co. Pte. Ltd. , 2004.

[53] H. Tezuka, "Mean Field Approximation to QCD", INS-Rep.-643 (1987).

[54] I. Lovas, W. Greiner, P. Hraskǿ and E. Lovas, Phys. Lett. B 156, 255 (1985).

[55] D. A. Fogaça, F. S. Navarra and L. G. Ferreira Filho, Phys. Rev. D 84, 054011 (2011).

[56] D. A. Fogaça, L. G. Ferreira Filho and F. S. Navarra, Nucl. Phys. A 819, 150 (2009).

[57] C. G. Bollini and J. J. Giambiagi, Rev. Bras. Fis. 17, 14 (1987).

[58] N. D. Minh, arXiv:0807.4431 [hep-th].

[59] K. Bhattacharya and P. B. Pal, Pramana 62 (2004) 1041. 
[60] K. Bhattacharya, "Solution of the Dirac equation in presence of an uniform magnetic field," arXiv:0705.4275 [hep-th].

[61] R. J. Furnstahl and B. D. Serot, Phys. Rev. C 41, 262 (1990).

[62] M. Strickland, V. Dexheimer and D. P. Menezes, Phys. Rev. D 86, 125032 (2012).

[63] V. Dexheimer, D. P. Menezes and M. Strickland, J. Phys. G 41, 015203 (2014).

[64] S. Chakrabarty, Phys. Rev. D 54, 1306 (1996).

[65] D. M. Paret and A. P. Martinez, arXiv:1010.0909 [astro-ph.HE].

[66] J. X. Hou, G. X. Peng, C. J. Xia and J. F. Xu, Chin. Phys. C 39, 015101 (2015).

[67] M. Chaichian, S. S. Masood, C. Montonen, A. Perez Martinez and H. Perez Rojas, Phys. Rev. Lett. 84, 5261 (2000).

[68] George B. Arfken, Hans J. Weber, "Mathematical Methods For Physicists", Fourth Edition, Academic Press , 1995.

[69] Site: http://mathworld.wolfram.com/ e software Wolfram Mathematica, versão 8.0 .

[70] P. N. Bogoliubov, Ann. Inst. Henri Poincaré 8, 163 (1967).

[71] A. Thomas and W. Weise, "The Structure of the Nucleon", Wiley, 2010.

[72] A. Chodos, R. L. Jaffe, K. Johnson, C. B. Thorn and V. F. Weisskopf, Phys. Rev. D 9, 3471 (1974).

[73] A. Chodos, R. L. Jaffe, K. Johnson and C. B. Thorn, Phys. Rev. D 10, 2599 (1974).

[74] M. Creutz, "Quarks, Gluons And Lattices”, Cambridge, Uk: Univ. Pr., 1983.

[75] C. Gattringer and C. B. Lang, Lect. Notes Phys. 788, 1 (2010). 
[76] S. Durr et al., Science 322, 1224 (2008).

[77] F. Csikor, G. I. Egri, Z. Fodor, S. D. Katz, K. K. Szabo and A. I. Toth, JHEP 0405, 046 (2004).

[78] Z. Fodor, S. D. Katz and K. K. Szabo, Phys. Lett. B 568, 73 (2003).

[79] C. R. Allton, S. Ejiri, S. J. Hands, O. Kaczmarek, F. Karsch, E. Laermann and C. Schmidt, Phys. Rev. D 68, 014507 (2003).

[80] C. R. Allton, M. Doring, S. Ejiri, S. J. Hands, O. Kaczmarek, F. Karsch, E. Laermann and K. Redlich, Phys. Rev. D 71, 054508 (2005).

[81] C. Bernard, C. E. DeTar, L. Levkova, S. Gottlieb, U. Heller, et. al., Phys.Rev. D 77014503 (2008).

[82] MILC Collaboration, S. Basak et. al., PoS LATTICE 2008, 171 (2008).

[83] C. DeTar, L. Levkova, S. Gottlieb, U. Heller, J. Hetrick, et. al., Phys.Rev. D 81 $114504(2010)$.

[84] T. Takaishi, P. de Forcrand, and A. Nakamura, PoS LATTICE 2009, 198 (2009).

[85] S. Borsanyi, G. Endrodi, Z. Fodor, S. D. Katz, S. Krieg, C. Ratti and K. K. Szabo, JHEP 1208, 053 (2012); Y. Aoki, G. Endrodi, Z. Fodor, S. D. Katz and K. K. Szabo, Nature 443, 675 (2006).

[86] S. Borsanyi, Z. Fodor, C. Hoelbling, S. D. Katz, S. Krieg and K. K. Szabo, Phys. Lett. B 730, 99 (2014).

[87] L. Landau and E. Lifchitz, "Fluid Mechanics", Pergamon Press, Oxford, (1987).

[88] A. Ghaani, K. Javidan and M. Sarbishaei, Astrophys. Space Sci. 358, 20 (2015).

[89] A. Ghaani and K. Javidan, arXiv:1712.10298 [hep-th]. 
[90] D. Martínez-Gómez, R. Soler and J. Terradas, APJ 832, 101 (2016); APJ 837, $80(2017)$.

[91] R. L. S. Farias, K. P. Gomes, G. I. Krein and M. B. Pinto, Phys. Rev. C 90, $025203(2014)$.

[92] G. Kowal, D. A. Falceta-Goncalves and A. Lazarian, New J. Phys. 13, 053001 (2011), M. S. Nakwacki, E. M. d. Gouveia Dal Pino, G. Kowal and R. SantosLima, J. Phys. Conf. Ser. 370, 012043 (2012).

[93] J. Y. Ollitrault, Eur. J. Phys. 29, 275 (2008).

[94] P. Romatschke, Int. J. Mod. Phys. E 19, 1 (2010).

[95] D. A. Fogaça, L. G. Ferreira Filho and F. S. Navarra, Phys. Rev. C 81, 055211 (2010).

[96] D. A. Fogaça, F. S. Navarra and L. G. F. Filho, Phys. Rev. C 88, 025208 (2013).

[97] D. A. Fogaça, H. Marrochio, F. S. Navarra and J. Noronha, Nucl. Phys. A 934, $18(2014)$.

[98] S. A. Balbus and J. F. Hawley, Astrophys. J. 376, 214 (1991).

[99] X. Wei, R. Hollerbach and A. Jackson, arXiv:1008.3706.

[100] H. M. Nussenzveig, "Causality and Dispersion Relations", Academic Press, (1972).

[101] S. Pu, T. Koide and D. H. Rischke, Phys. Rev. D 81, 114039 (2010).

[102] G. K. Vallis, "Atmospheric and oceanic fluid dynamics: fundamentals and largescale circulation", Cambridge University Press, page 238 to page 240 (2006).

[103] Y. Minami and Y. Hidaka, arXiv:1401.0006 [hep-ph].

[104] Korteweg, D. J., de Vries, G., Phil. Mag. 39, 422443 (1895). 
[105] G. N. Fowler, S. Raha, N. Stelte and R. M. Weiner, Phys. Lett. B 115, 286 (1982).

[106] D. A. Fogaça and F. S. Navarra, Phys. Lett. B 639, 629 (2006).

[107] C. Plumberg and J. I. Kapusta, Phys. Rev. C 95, 044910 (2017).

[108] M. Nahrgang, M. Bluhm, T. Schaefer and S. Bass, arXiv:1704.03553.

[109] G. Wilk and Z. Wlodarczyk, arXiv:1701.06401.

[110] Y. Akamatsu, A. Mazeliauskas and D. Teaney, Phys. Rev. C 95, 014909 (2017).

[111] J. I. Kapusta, B. Müller and M. Stephanov, Phys. Rev. C 85, 054906 (2012).

[112] H. Washimi and T. Taniuti, Phys. Rev. Lett. 17, 996 (1966).

[113] R.C. Davidson, "Methods in Nonlinear Plasma Theory", Academic Press, New York an London, (1972).

[114] H. Leblond, J. Phys. B: At. Mol. Opt. Phys. 41, 043001 (2008).

[115] A. Ghaani and K. Javidan, arXiv:1712.10298 [hep-th].

[116] D. A. Fogaça, F. S. Navarra and L. G. Ferreira Filho, inWilliams, Matthew C.: Solitons: Interactions, Theoretical and Experimental Challenges and Perspectives, 191-256, ISBN:-978-1-62618-234-9.

[117] V. O. Vakhnenko, E. J. Parkes, Nonlinearity 11, 1457 (1998).

[118] Y. A. Stepanyants, Chaos Solitons Fractals 28, 193 (2006).

[119] E. Yusufoğlu, A. Bekir, Applied Mathematics and Computation 186, 256 (2007).

[120] E. J. Parkers, Chaos Solitons Fractals 31, 602 (2007).

[121] E. R. Johnson, R. H. J. Grimshaw, Phys. Rev. E 88, 021201 (2013). 
[122] R. G., D. Pelinovsky, Discrete and Continuous Dynamical Systems 34, 557 (2014).

[123] R. Grimshaw, D. Pelinovsky, Discrete and Continuous Dynamical Systems 34, 557 (2014).

[124] Bao-Feng Feng, Ken-ichi Maruno and Yasuhiro Ohta, Journal of Physics A: Mathematical and Theoretical 50, 055201 (2017).

[125] L. A. Ostrovsky, Oceanology 18, 119 (1978).

[126] Norman K. Glendenning, "Compact Stars, Nuclear Physics, Particle Physics and General Relativity", 2nd ed. (Springer-Verlag, New York, 2000).

[127] Max Planck Institute for Physics. (2012, March 28). ScienceDaily. Retrieved June 12, 2018 from www.sciencedaily.com/releases/2012/03/120328090850.htm.

[128] E. Farhi, R.L. Jaffe, Phys. Rev. D 30, 2379 (1984).

[129] J. R. Oppenheimer and G. M. Volkoff, Phys. Rev. 55, 374 (1939).

[130] P. Demorest, T. Pennucci, S. Ransom, M. Roberts and J. Hessels, Nature 467, $1081(2010)$.

[131] M. H. van Kerkwijk, R. Breton and S. R. Kulkarni. Astrophys J. 728, 95 (2011).

[132] J. Antoniadis et al., Science 340, 6131 (2013).

[133] J. R. Oppenheimer and G. M. Volkoff, Phys. Rev. 55, 734 (1939).

[134] R. C. Tolman, Phys. Rev. 55, 364 (1939).

[135] N. Glendenning, Compact stars, (Springer-Verlag, New York, 2000).

[136] L. Paulucci, E. J. Ferrer, V. de la Incera and J. E. Horvath, Phys. Rev. D 83, 043009 (2011). 
[137] J. Rafelski and J. Birrell, J. Phys. Conf. Ser. 509, 012014 (2014).

[138] W. Florkowski, Nucl. Phys. A 853, 173 (2011).

[139] U. Ornik and R. M. Weiner, Phys. Rev. D 36, 1263 (1987).

[140] T. Kalaydzhyan and E. Shuryak, Phys. Rev. D 91, 083502 (2015).

[141] K. Yagi, T. Hatsuda, Y. Miake, Camb. Monogr. Part. Phys. Nucl. Phys. Cosmol. 23, 1 (2005).

[142] G. L. Guardo, V. Greco and M. Ruggieri, AIP Conf. Proc. 1595, 224 (2014).

[143] U. Ornik and R. M. Weiner, Phys. Rev. D 36, 1263 (1987).

[144] B. Friman, C. Hohne, J. Knoll, S. Leupold, J. Randrup, R. Rapp and P. Senger, Lect. Notes Phys. 814, 980 (2011).

[145] theor.jinr.ru/twiki-cgi/view/NICA/NICAWhitePaper; K. A. Bugaev, arXiv:0909.0731 [nucl-th] e referências.

[146] G. Lugones, Eur. Phys. J. A 52, 53 (2016).

[147] H. Heiselberg, C. J. Pethick and E. F. Staubo, Phys. Rev. Lett. 70, 1355 (1993); J. E. Horvath, Phys. Rev. D 49, 5590 (1994); J. E. Horvath, O. G. Benvenuto and H. Vucetich, Phys. Rev. D 45, 3865 (1992).

[148] D. Logoteta, C. Providencia, I. Vidana and I. Bombaci, Phys. Rev. C 85, 055807 (2012); D. Logoteta, I. Bombaci, C. Providencia and I. Vidana, Phys. Rev. D 85, 023003 (2012); I. Bombaci, D. Logoteta, C. Providencia and I. Vidana, Astron. Astrophys. 528, A71 (2011).

[149] I. Bombaci, I. Parenti and I. Vidana, Astrophys. J. 614, 314 (2004).

[150] M. L. Olesen and J. Madsen, Phys. Rev. D 49, 2698 (1994). 
[151] L.P. Csernai and J.O. Kapusta, Phys. Rev. Lett. 69, 737 (1991); Phys. Rev. D 46, 1397 (1992).

[152] G. Neergaard and J. Madsen, Phys. Rev. D 62, 034005 (2000); Phys. Rev. D 60, 054011 (1999); I. Mardor and B. Svetitsky, Phys. Rev. D 44, 878 (1991).

[153] D. Kroff and E. S. Fraga, Phys. Rev. D 91, 025017 (2015); A. F. Garcia and M. B. Pinto, Phys. Rev. C 88, 025207 (2013); M. B. Pinto, V. Koch and J. Randrup, Phys. Rev. C 86, 025203 (2012); L. F. Palhares and E. S. Fraga, Phys. Rev. D 82, 125018 (2010).

[154] T. Biro, H. W. Barz, B. Lukacs and J. Zimanyi, Phys. Rev. C 27, 2695 (1983); H. W. Barz, B. L. Friman, J. Knoll and H. Schulz, Phys. Lett. B 242, 328 (1990); I. N. Mishustin, Phys. Rev. Lett. 82, 4779 (1999); I. N. Mishustin and O. Scavenius, Phys. Rev. Lett. 83, 3134 (1999); O. Scavenius, A. Dumitru, E. S. Fraga, J. T. Lenaghan and A. D. Jackson, Phys. Rev. D 63, 116003 (2001); J. Randrup, Phys. Rev. Lett. 92, 122301 (2004); G. Torrieri, B. Tomasik and I. N. Mishustin, Phys. Rev. C 77, 034903 (2008).

[155] L. P. Csernai, I. N. Mishustin, Phys. Rev. Lett. 74, 5005 (1995).

[156] J. J. Bjerrum-Bohr, I. N. Mishustin and T. Dossing, Nucl. Phys. A 882, 90 (2012).

[157] J. J. Bjerrum-Bohr, I. N. Mishustin and T. Dossing, Nucl. Phys. A 923, 19 (2014).

[158] Lord Rayleigh, Phil. Mag. 34, 94 (1917).

[159] Como exemplo: http://authors.library.caltech.edu/25021/1/chap4.pdf

[160] H. T. Elze, Y. Hama, T. Kodama, M. Makler and J. Rafelski, J. Phys. G 25, 1935 (1999). 
[161] Kohsuke Yagi, Tetsuo Hatsuda and Yasuo Miake "Quark-Gluon Plasma: From Big Bang to Little Bang", Cambridge University Press; First edition, 2008.

[162] N. A. Kudryashov, D. I. Sinelshchikov, Phys. Lett. A 379, 798 (2015).

[163] W. N. Bailey, "Generalized hypergeometric series (Cambridge tracts in mathematics and mathematical physics No. 32)", Cambridge University Press; First edition, 1935; W. Magnus, F. Oberhettinger, "Formulas and Theorems for the Functions of Mathematical Physics", Chelsea Publishing Company N.Y., 1954; http://mathworld.wolfram.com/HypergeometricFunction.html.

[164] A. L. Espindola and D. P. Menezes, Phys. Rev. C65, 045803 (2002); A. M. S. Santos and D. P. Menezes, Braz. J. Phys. 34, 833 (2004).

[165] K. Rajagopal and N. Tripuraneni, JHEP 1003, 018 (2010).

[166] M. Habich and P. Romatschke, JHEP 1412, 054 (2014). 